\title{
Management information for LAMPF
}

\author{
R. A. Jameson \\ R. S. Mills \\ M. D. Johnston
}


This report was prepared as an account of work sponsored by the United States Government. Neither the United States nor the United States Atomic Energy Commission, nor any of their employees, nor any of their contractors, subcontractors, or their employees, makes any warranty, express or in)plied, or assumes any legal liability or responsibility for the accuracy, completeness or usefulness of any information, apparatus, product or pracess disclosed, or represents that its use would not infringe privately owned rights.

In the interest of prompt distribution, this LAMS report was not edited by the Technical Information staff.

Printed in the United States of America. Available from

National Technical Information Service

U. S. Department of Commerce

5285 Port Royal Road

Springfield, Virginia 22151

Price: Printed Copy $\$ 5.45$; Microfiche $\$ 1.45$ 
MANAGEMENT INFORIAATION FOR LAMPF

by

R. A. Jameson, R. S. ilills, and M. D. Johnstan

ABSTRACT

Various types of information are required on a continuing basis for the operation, maintenance, scheduling, budgeting. and general support of a large acceleraton facility. 'Ioderi general purpose file management and information retrieval systems allow the operating division to concentrate on its information nieds with minimal programming investment. This report covers the operational aspects of various data-bases and the associated iriformation retrieval and reporting nos' in use and under development.

\section{INTRODJCTION}

This report outlines the status of the development of computer-based tools proposed as an adjunct to the operation as a production facility of the Clinton P. Andersoin Meson ?hys ics Facility at Los Alamos, ivew Mexico (LAltPF).

A continuing flow of information is required to plan, coordinate, and evaluate the activities of an operational facility, especially in the four areas of maintenance, scheduling, budgeting, and general support to users.

The problems of storing, retrieving, analyzing, and displaying information have beel greatly aided in recent years by the development of genera?ized data-base management software which allow the operating division to concentrate on its information needs with a minimum pronramming investment. At LASL, a computer program called "Generalized Information Retrieval, LASL System" (GIRLS) ${ }^{2}$ has been available. This is a general purpose file management and information retrieval system which runs on the $\operatorname{CDC} 6600 / 7600$ computers. It is designed with English language commands to be used without knowledge : if programming. With it, one can structure a data-base and spec,fy retrieval problems quickly.
The standard formats provided by GIRLS for input and output are very simple, line-by-line structures. For many applications, specific columnar input formats, graphical outputs, etc., are desired. These must be progranmed by the user, but are specific to his particular needs. This report covers the applications for which GIRLS, plus this special programing, have been used. Approximately two manyears have been spent on system andlysis and programming. Considerable other help was used on a shortterm basis to gather inforination. The maintenance of the present data-bases occunies approximately 3 full-time equivalents, spread over about 16 people.

The usefulness of the results to date have resulted in the establishment of a solid foundation and approach, and in recent decisions to expand the effort to other areas and to support production use. A]so on the horizon are more powerful data-base management tools which will be supported and used Laboratory-wide. A system is presently being evaluated which would run in a time-shared mode, allowing the user to work interactively with his data. Many of the input-output requi rements which required user coding up to now wi 11 be included in ceneralized form in the new system, along with more fltxible and 
extensive data-handling options. As will be seen in the following, many areas of interest have overlapping data requirements; the capability for crossreferencing in the newer systems is a great advantage. The whole field is undergoing rapid development and presents enormous potential as an aid to the operation of a modern facility.

The Appendixes present the operational aspects of the various data-bases and assnciated input and outputs so far in use and under divelopment. Appendix $A$ includes program listings which show detailed data-base descriptions. Appendix $B$ contairs examples of pre- and post-processor programs which have been written.

\section{It:- OPERATIONS/MAINTENANCE \\ A. Inventory}

The initial step was to deine what types of equipment had to be handled. It was decided to classify by type rather than by individual piece in order to make the system manageable and because management information concerning types shouid be sufficient. For instance, there may be 50 identical amplifiers classified as one type. The words "unit" and "item" are synonyms for type. At this point, all equipment from the injectors to the end of the accelerator has been inventoried, organized into system and functi unal groupings, and cross-referenced to location. A unit number was assigned io each type and labeis were affixed to the equipment. There are about 600 categories in the inventory so far; the system will eventually be extended to the switchyard and beam-line equipment. Table I shows the inventcry format; it has a three-level hierarchical structure.

B. Background Questionnaire

The second step was to set up a questionnaire to find out pertinent information on each unit. A sample form is shown in Fig. 1 . This information was set up in the form of a data-base using the GIRLS program. The stored data for Unit Number 61, in the Controller function of the $r f$ system, is shown in the standard GIRLS output format in Fig. 2.

A great deal of potential is implied in the background data-base. It can be used as a framework for completing development in a number of directions; for example, development of maintenance and system use procedures, drawing updates, preventive mainte- nance program development and scheduling, spare parts inventory control, definition of personne? requirements, and so $\mathrm{cn}$. Information retrieval can be set up in many different ways as needed. The definition which it can provide, along with the operating history described below, can be used eventually as input for investigation of overall reliability and for system modeling. (Appendix A.1)

C. Daily Reporting

A system for daily trouble reporting and maintenance action reporting was then set up. Information is received on the card format indicated in Fig. 3. A great deal of thought went into this simple card. The main criterion was to make it simple to use, so that it wculd be used. This meant that the information requested had to be distilled to the minimum feasible amount. The information was to serve two purposes. First, immediate communication is necessary to the maintenance staff, who needs to :now what needs repair, where it is, and enough of the symptoms to get started on trouble-shooting. The second requirement is long-range in terms of operationa? history. In this case, management really needs only enough information to be able to notice trends and to have some systematic measurement capability on an overall basis. The cards are distributed liberally throughout the facility. In many cases, the action is completed on-line; in others, the maintenance personnel finish the report on the yellow copy. The cards are gathered daily, and a daily report is prepared, using a LAMPF PDP-11 data acquisition compu-. ter for quick turnaround. This computer contains an abbreviated dictionary which matches the unit number to the unit name, system, and responsible system personnel. Examples are presented in Fig. 4.

D. Sumaries of Dperating History

The reports are then stored in a data-base for long-term use. Eventually it will be possible to obtain statistical data on trends and varjous categories of reliability. For the present, graphical summaries are prepared on a monthly basis. The programming for these summaries was dane separately, using the information retrieved from the GIRLS database. Figure 5 shows the information stored for Unit No. 61. In Fig. 6, Unit 67's history over ? month is summarized under its functional heading of Controller, with cross-reference to location. In Fig. 7, the controller function is summarized under 
TABLE I

MAINTENANCE BACKGROUND DATA BASE - INVENTORY

GENERAL OUTLINE

\begin{tabular}{|c|c|c|c|}
\hline System & Function & Numbers & Nature of Items \\
\hline \multirow[t]{5}{*}{ DAC } & MAST-TIME & 5300,530 & Master-Timer in 805,201 , transport, and injector \\
\hline & VIOEO & 5100 & Video bins: sector and module types \\
\hline & RICE & 5200 & Rice components - throughout acce?erator \\
\hline & ADS & 5000,500 & Analog data chassis; sample and hold bins and components \\
\hline & LIGHT-LINK & 520 & Light link transmitters, receivers and related equip, in injector \\
\hline \multirow[t]{6}{*}{ DIST } & PROTECTION & 7000,700 & Fast protect equipment; RUi permissive equipment \\
\hline & RAD-MON & 7300 & Radiation monitor, beam spill and activation protect equipment \\
\hline & COMM & 7400 & Communications and interphone stations \\
\hline & TV & 7200 & Television cameras, monitors, amplifiers, etc. \\
\hline & SAFETY & 7100,710 & Personnel safety components; time delay equipment \\
\hline & FACILITY & 7500 & Accelerator racility status AIM bin \\
\hline \multirow[t]{3}{*}{ VACUUM } & ION-PUMP & 6000,600 & Vacuum ion pump, PS, ion gauges, start supplies \\
\hline & CONTROL & & Vacuum valves, switches, control bins and components \\
\hline & SFT-VAC & & Soft vacuum equipment in 201 \\
\hline \multirow[t]{3}{*}{ WATER } & CONTROL & 8000,800 & $\mathrm{H}_{2} \mathrm{O}$ bins, valve asseliblies \\
\hline & PROTECTION & & Flow switch \\
\hline & PLUMBING & & $\mathrm{H}_{2} \mathrm{O}$ cabinets \\
\hline \multirow[t]{3}{*}{ BEAM } & MAGNET & $2000=805 \mathrm{MHz}$ & Quad, steering, bending magnets, power supplies, bins, and controls \\
\hline & MONITOR & $\begin{aligned} 200 & =201 \mathrm{MHz} \\
300 & =\mathrm{TR} \\
400 & =\mathrm{XPRT}\end{aligned}$ & $\begin{array}{l}\text { Linear actwators, drivers, emittance jaws, collectors, etc. } \\
\text { Beam current and position monitor equip.; wire scan gear }\end{array}$ \\
\hline & POWER & & Standard $24 \mathrm{~V}$ PS, used for much equipment in the racks \\
\hline \multirow[t]{11}{*}{ RF } & HV & $\begin{aligned} 1000 & =805 \mathrm{MHz} \\
900 & =\text { Source }\end{aligned}$ & High voltage power supplies, meters, controls, HV pads, cap. rooms \\
\hline & $A C-D C-D I S T$ & $\begin{aligned}<200 & =201 \mathrm{MHz}\end{aligned}$ & Sector and module $A C$ and $D C$ distribution system \\
\hline & $H I-P W R-R F$ & & $\begin{array}{l}\text { Klystrons, associated maynets, suppiies and controls } \\
\text { Power amplifiers, supplies and controls }\end{array}$ \\
\hline & INT-PWR-RF & & Intermediate power amplifiers and associated supplies (201-MHz) \\
\hline & RF-REF & & Source room RF reference equipment \\
\hline & $\begin{array}{l}\text { RF-AMP-805 } \\
\text { RF-AMP-201 }\end{array}$ & & $\begin{array}{l}\text { Source room amplifiers, power supplies, filament } \\
\text { supplies for } 805 \text { and/or } 201 \mathrm{MHz}\end{array}$ \\
\hline & PROTECTION & & $\begin{array}{l}\text { Crowbar logic; duty factor interlock; module control units } \\
\text { Fault protect equipment; pulse delays }\end{array}$ \\
\hline & CONTROLLER & & $\begin{array}{l}\text { Phase and amplitude controls; feed forward amps, preamps } \\
\text { Waveguide couplers; resonance and drive line temp controls; } \\
\text { tuning controls }\end{array}$ \\
\hline & MODULATOR & & $\begin{array}{l}\text { Reference line; interface amplifiers and PS; electronic phase } \\
\text { shifters } \\
\text { Kiystron, PA, and IPA modulators; status panel }\end{array}$ \\
\hline & AIR & & Rack ventilators and blowers \\
\hline & DELTA-T & & A11 delta-time equipment for phase and amplitude measurements \\
\hline \multirow[t]{4}{*}{ INJECTOR } & $\mathrm{C}-\mathrm{W}$ & 4000 & Cockcroft-walton amplifiers, power supplies, generators \\
\hline & ION-SOLRCE & & Ion-source equipment in $\mathrm{H}^{+}$and $\mathrm{H}^{-}$domes \\
\hline & CONTROL & & Controls for injectors; computer equipment in ICR \\
\hline & PROTECTION & & Protective devices cor injector equipment \\
\hline
\end{tabular}


its rf system heading, and in Fig. 8, the rf system is summarized with the other major systems. Figures 9, 10, and 11 show the summaries graphically over a year-long period. Figure 12 is a summary by location, cross-referenced to unit number. Recent extensions wit7 provide for storage of scheduled operating time and calculation of MTBF (mean time before failure) and availability at each hierarchical level.

The card format has changed from a two-card system, one each for operations and maintenance, to the present one-card approach. Information has been collected for approximately 6 months, and as operators and maintenance personnel have received experience and training, the data-base is reaching adequate reliability. (Appendix A.2 and A.3)

\section{WORK ACCOUNTING}

Figure 13 is a form on which work requests can be written. Figure 14 shows an example of a typical work request and subsequent actions. Figures 15 and 16 consist of a week $1 y$ and quarterly summary. A nested sorting capability provided by the GIRLS program for retrieved information is evidenced in these summaries. The items are listed according to the group from whom the work is requested, and sorted according to the date required. The basic idea behind this preliminary data-base was just to get needed work written down, with indication of the interfaces involved. This step must precede scheduring. A great deal of thought and work is still needed before effective scheduling tools become a reality. (Appendix A.4)

\section{PROPERTY ACCOUNTING}

A. Capital Equipment

Figure 17 exhibits the format for the capital equipment property control data-base. Approximately 10,000 items are now controlled in this manner. (Appendix A.5)

B. LEEP and MP-Pool Equipment Inventory Control

Two pools of equipment, MP-Pool and LAMPF Electronic Equipment Pool (LEEP), have been formed to support in-house research and to support the experimental programs involving outside users. Figures 18 and 19 cover information retrieval formats. This equipment must be scheduled to match the rapidly changing experimental program. Generalized scheduling tools would be a great aid and will be a topic for future work. For the present, the pool equipment is being allocated with the aid of a specialized Fortran program which uses information retrieved from the GIRLS data-base. (Appendix A.6)

\section{EXPERIMENTAL PROGRAM SUPPORT \\ A. LAMPF Users}

The users liaison office maintains records about the neariy 1000 members from some 300 institutions. The data-base is used to provide mailing lists and labels, and listings of interests, institutions, nationalities, etc. Additional statisticai reports are provided periodically for the AEC. Figures 20 and 21 show examples of printouts programmed from selected retrieves. (Appendix A.7)

\section{B. Experirant Scheduling}

There currently are 115 approved proposals involving 369 participants for the experimental program at LAMPF. An additional 60 proposals have been submitted for consideration. The beam channels to be used, hours of beam time allocated, dates scheduled, and related information are processed through a GIRLS data-base. Mailing labels can be made automatically from this data-base and the LAMPF users data-base above. Figure 22 shows information stored concerning a typical experiment. Figures 23 and 24 display two types of summaries retrieved from the data-base. (Appendix A.8)

\section{GENERAL SUPPORT}

A. Office and Lab Space

The arrival of users at LAMPF requires that space assignments be managed to accommodate both temporary and permanent occupants. (Appendix A.9) B. Summary

In summary, it should be clear that there are many possibilities. Extersions to budgeting, material accolinting and other areas are now being considered.

\section{REFERENCES}

1. R. A. Jameson, "Management information for LAMPF," Internal Report, Los Alamos Scientific Laboratory, August 3, 1972.

2. W. Draisin and G. M. Connor, "Guide to GIRLS Vol. 1, User's Manual, "Internal Report, Los Alamos Scientific Laboratory, June 1, 1973. 


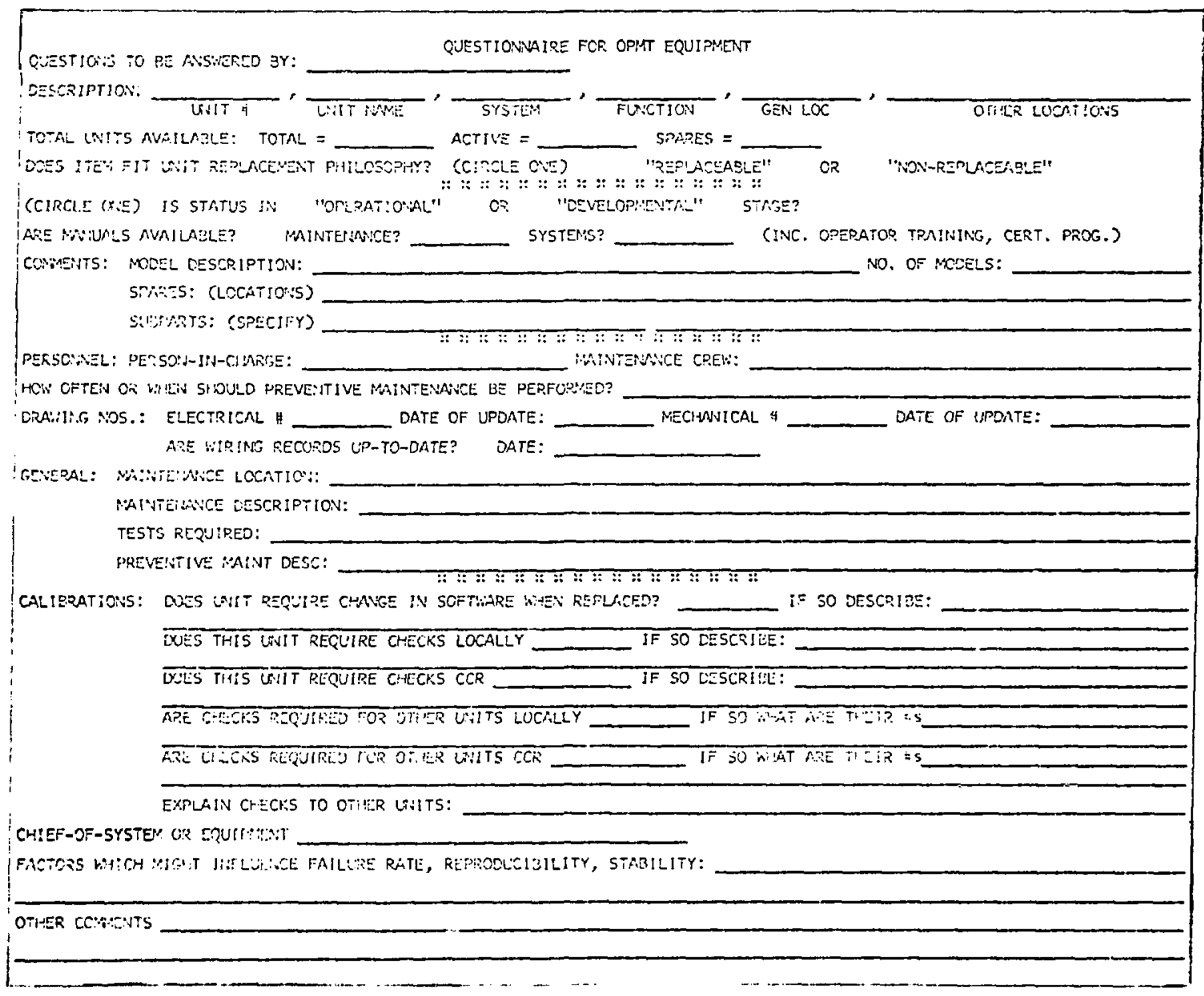

Fig. 1. Questionnaire on accelerator equipment to catalog information concerning operation and maintenance. (Appendix A.1) 


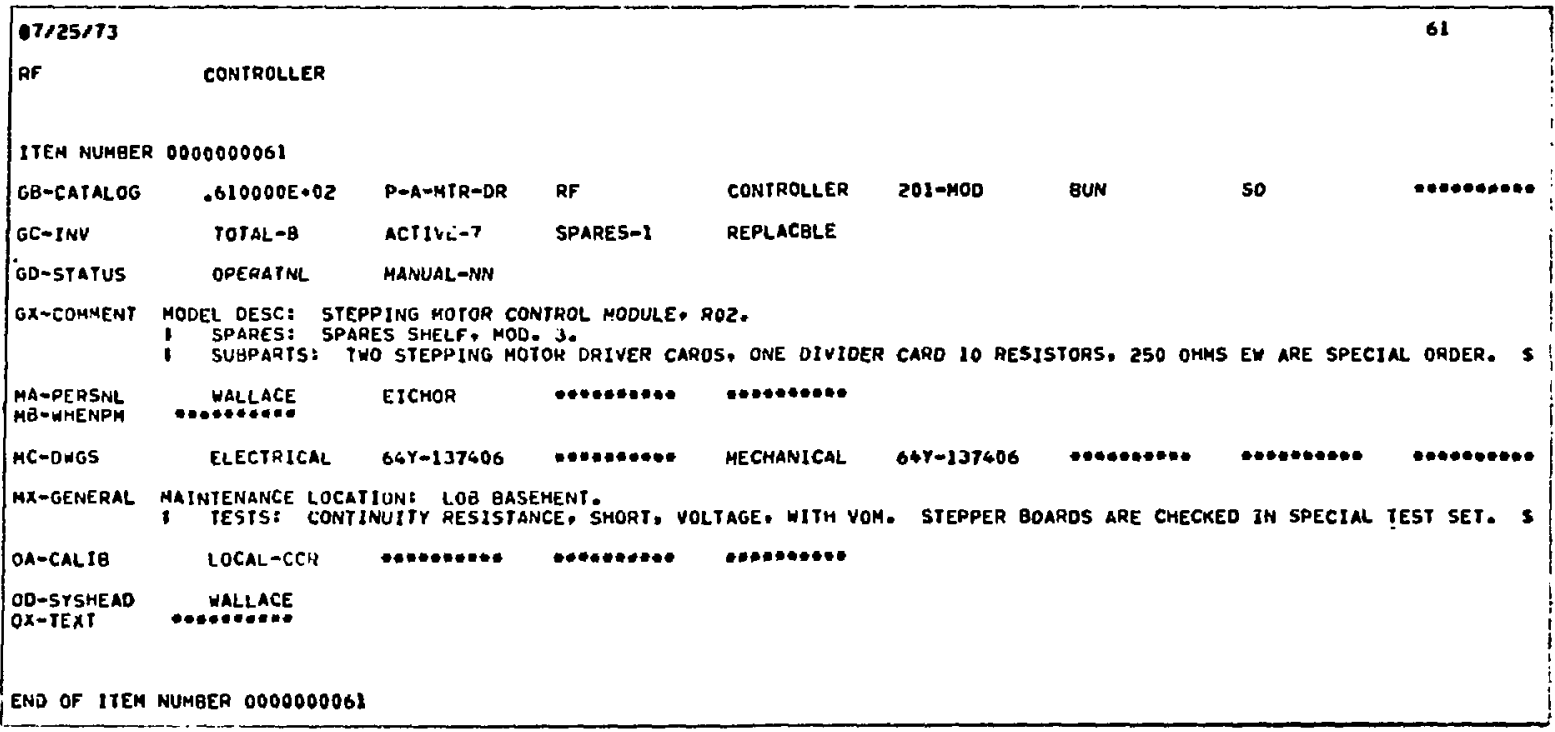

Fig. 2. Computer report format for unit questionnaire information stored in the Background data-base. Unit 61 will be developed as an examp10. (Appendix A.1) 


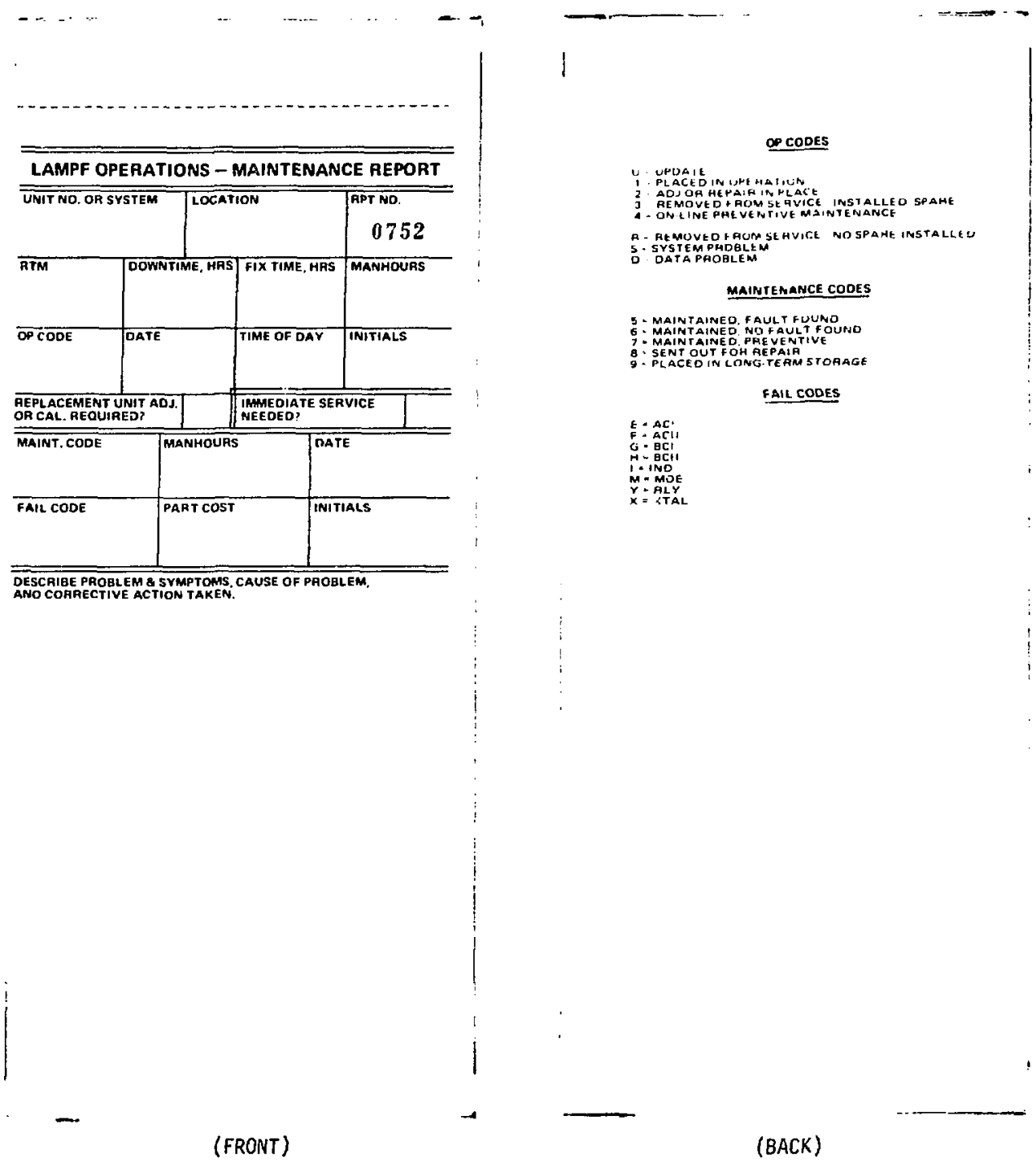

Fig. 3. Reporting card for actions taken on equipment. Card is same size as an IBM card and is in two copies - a white top slip and a stiff yellow back with carbon between. White copy is turned in; yellow cony is attached to equipment when appropriate.

RTM = running time meter

Downtime $=$ time lost on primary scheduled objective

Fix Time = time taken to fix or replace; can be same as downtime

Manhours $=$ (fix time) $\times$ (people involved)

Op Code = a simple breakdown for computer coding, printed on back of card

Replacement. Unit Adj. or Cal. Required? = to be checked when on-line adjustment or calibration is required, either on unit replaced or other units affected. Can be correlated with background data-base and used to reinforce training.

Fail Code $=$ kept very simple on purpose. Presently identifies certain standardized modules.

Comments $=$ left free-form on purpose. 
Fig. 4. Examples from the daily operations report and the maintenance report, which is generated once or twice weekTy. (Appendix B.4.b)

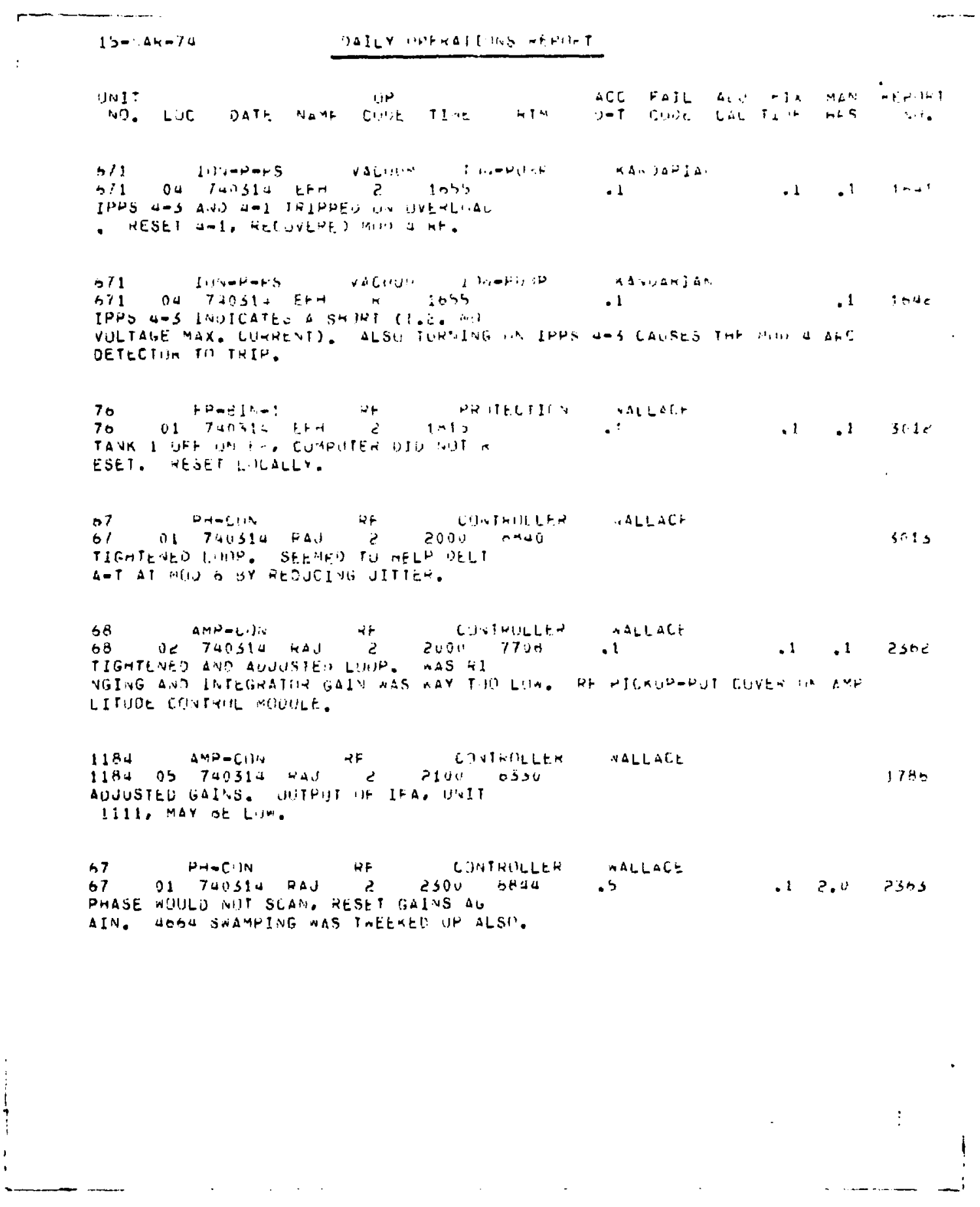

continued 


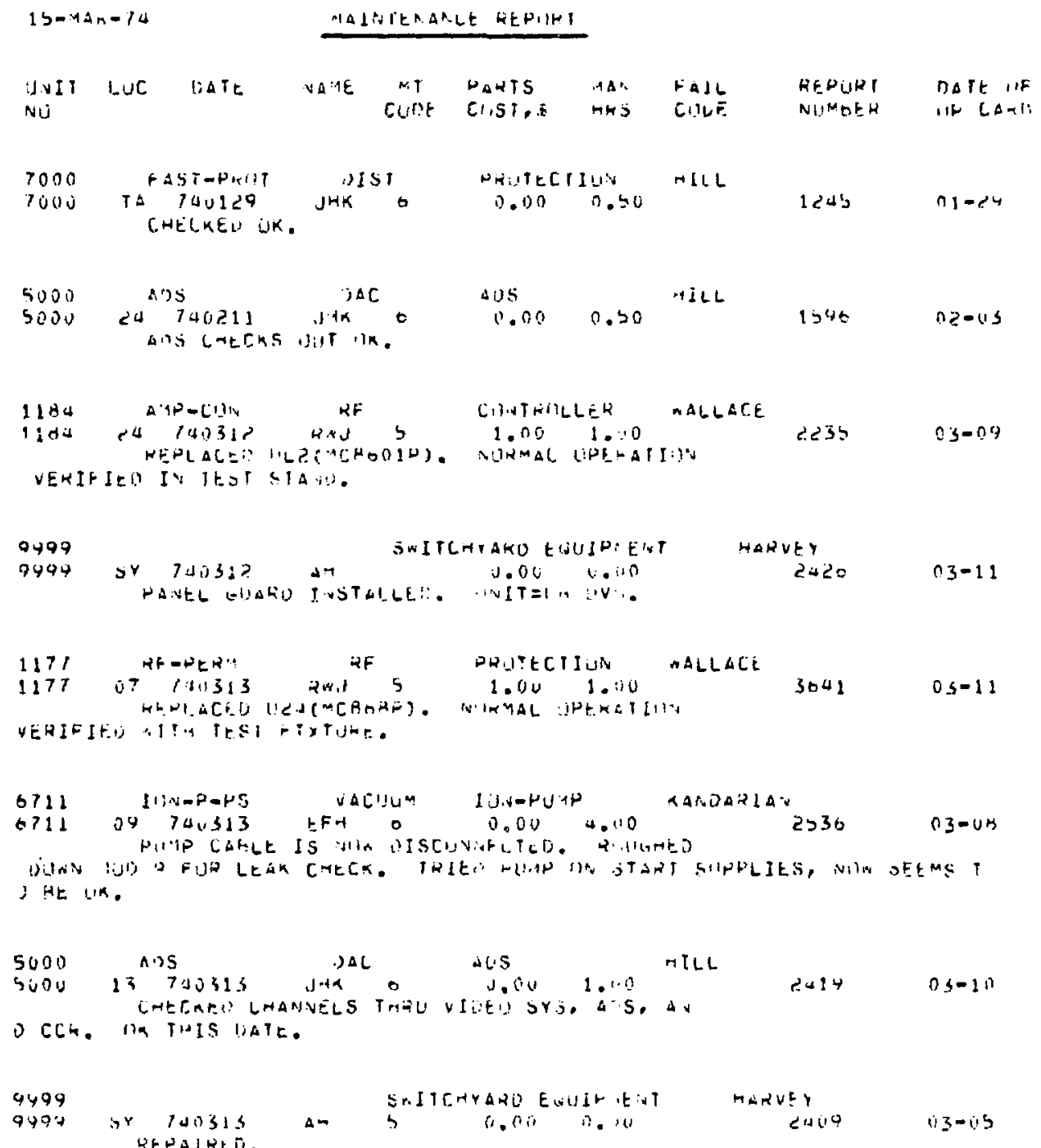




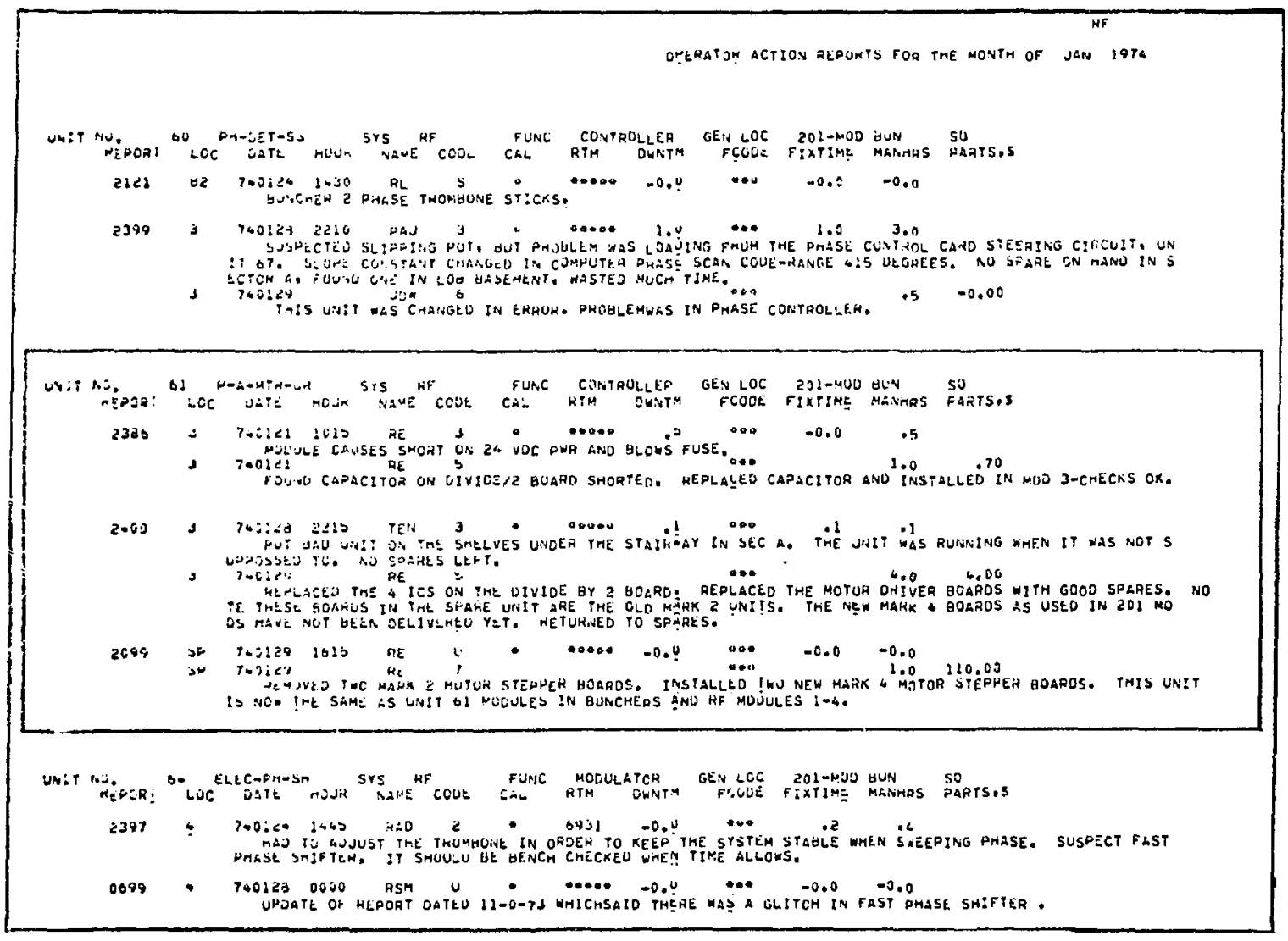

Fig. 5. Detailed listing of actions regarding Unit 61 for January 1974. (Appendix A.2, B.1.a, B.2.a) 


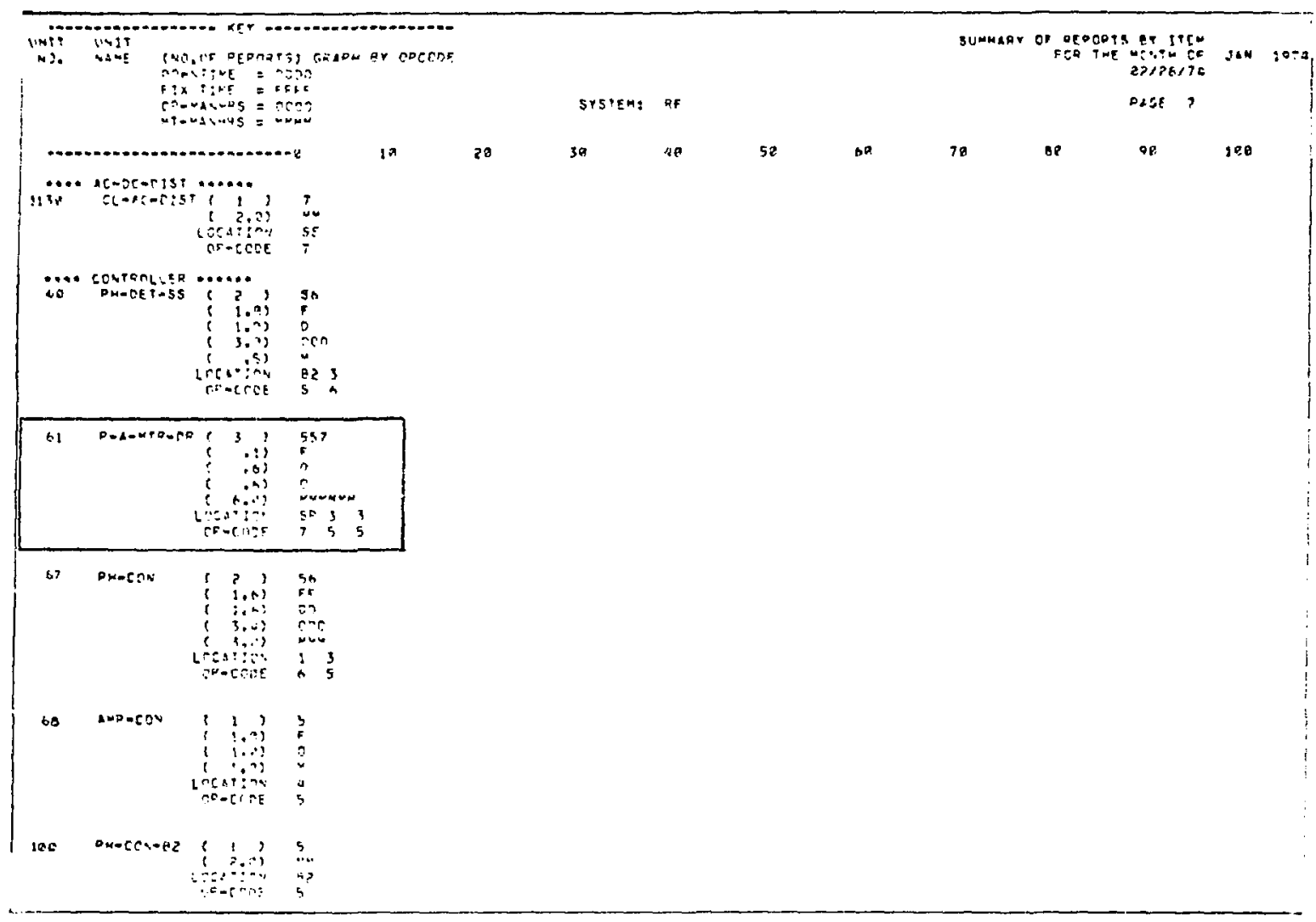

Fig. 6. Summary of Unit 61 actions, under the Controller functional heading of the RF system. (Appendix A.2) 


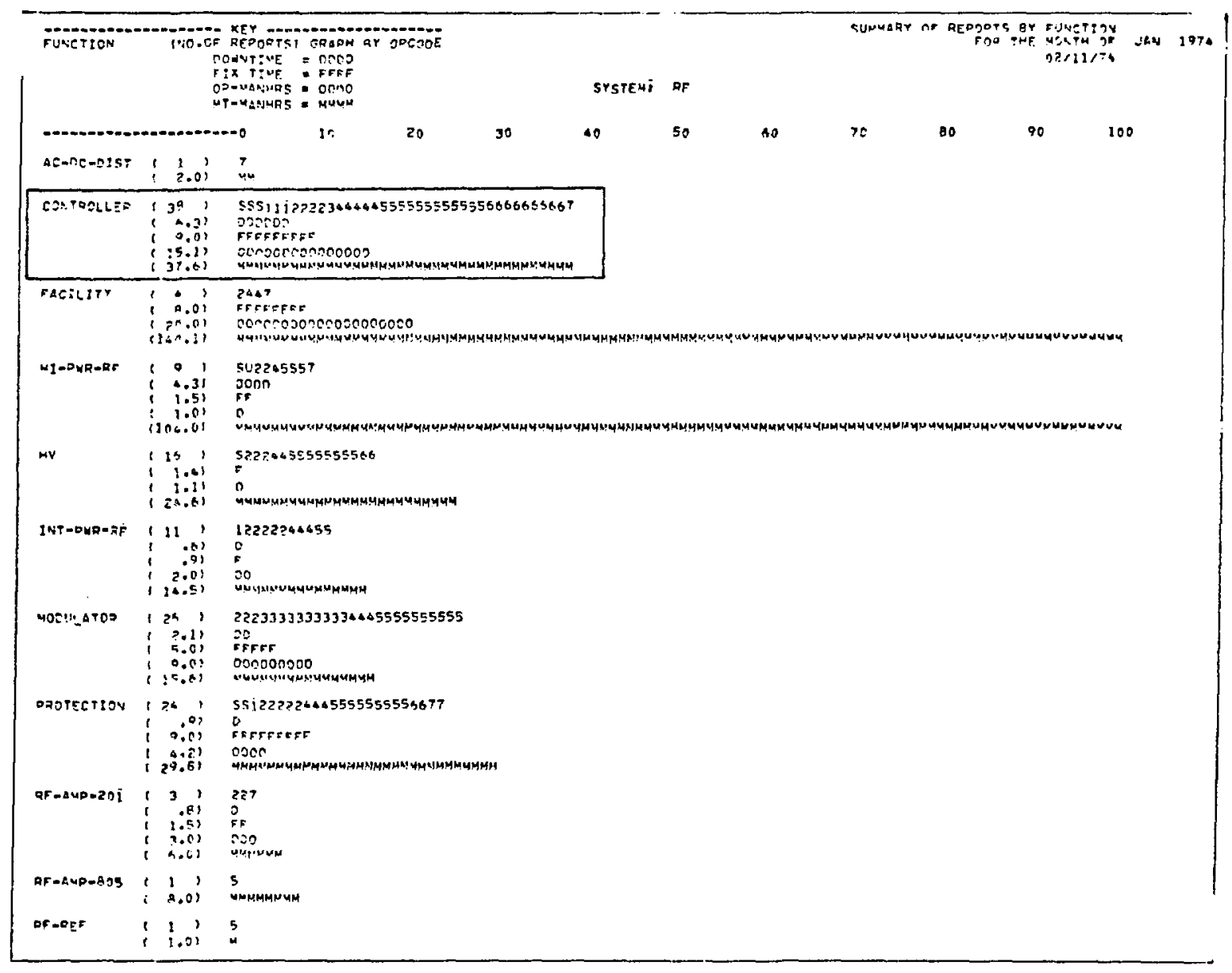

Fig. 7. Controller function summary, along with other functional headings under the RF system. (Appendix A.2, B.3.a) 


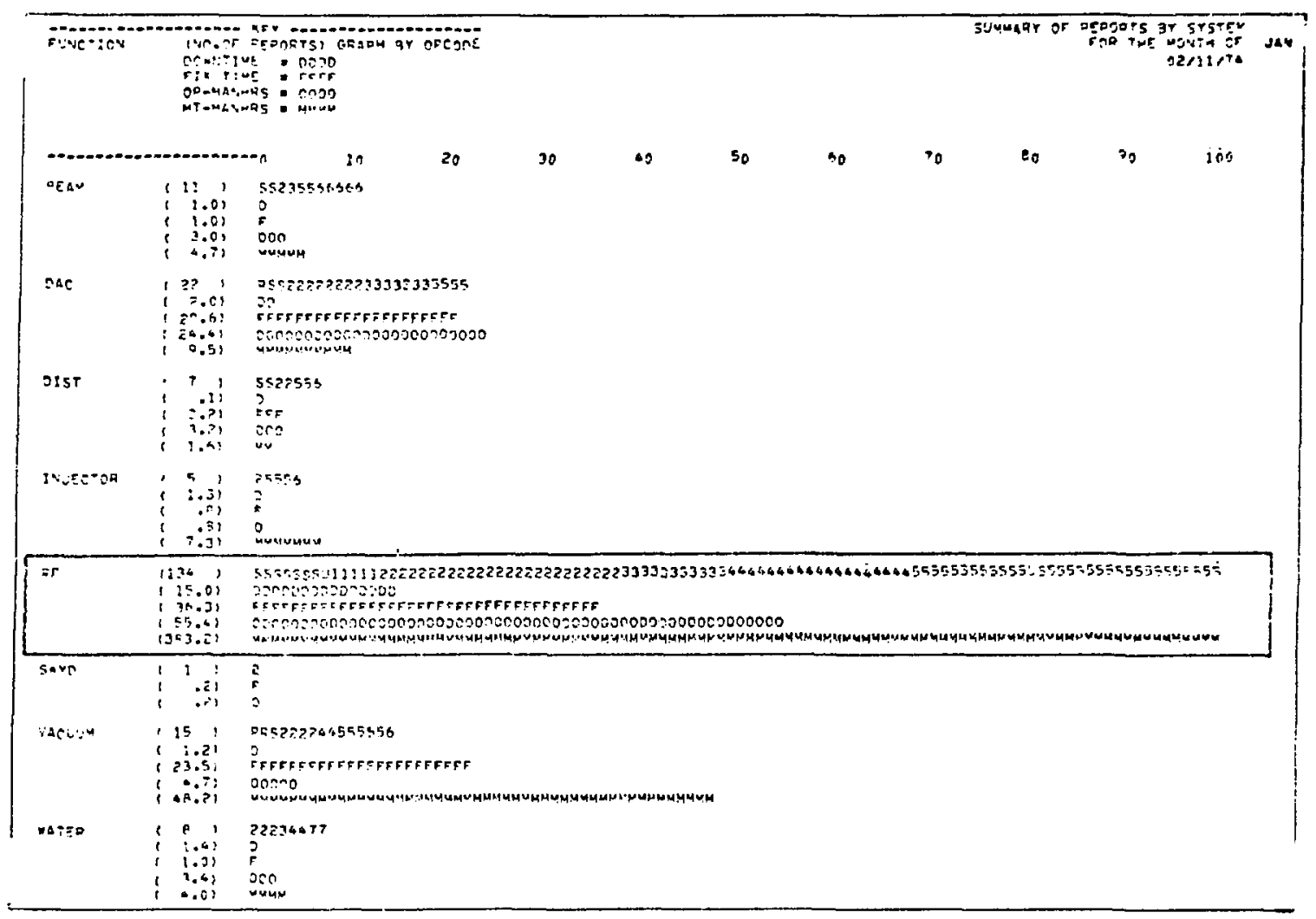

Fig. 8. RF system summary, along with other systems. (Appendix A.2, B.3.a)

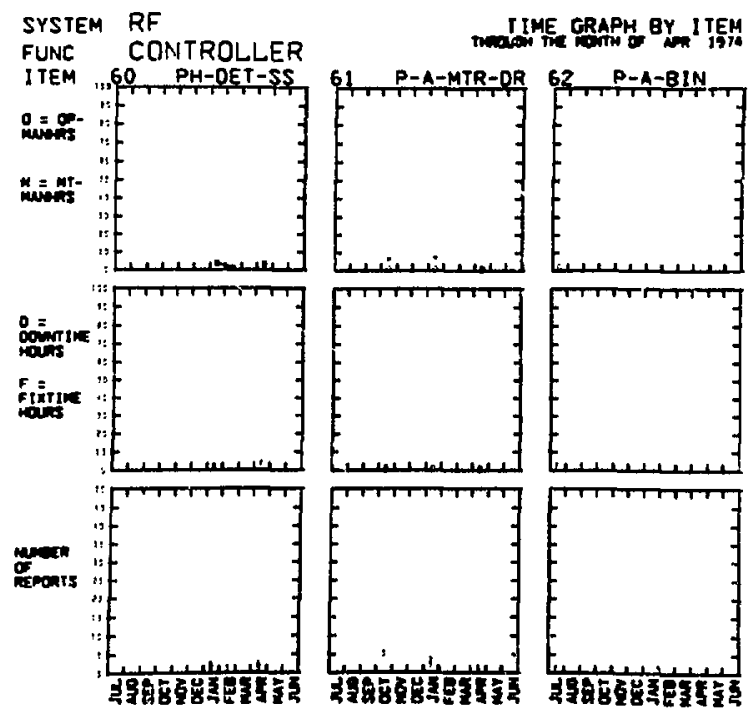

Fig. 9. Running summary of Unit 61 fron July 1973. The reports are cross-referenced to location by op code. (Appendix A.3, B.3.a)

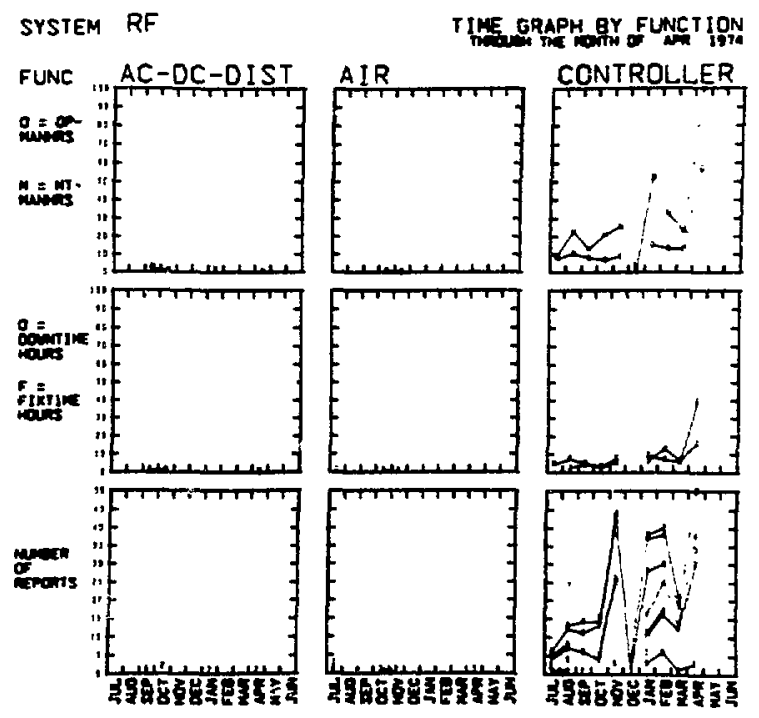

Fig. 10. Running summary of the Controller function of the RF system. (Appendix A.3, B.3.a) 


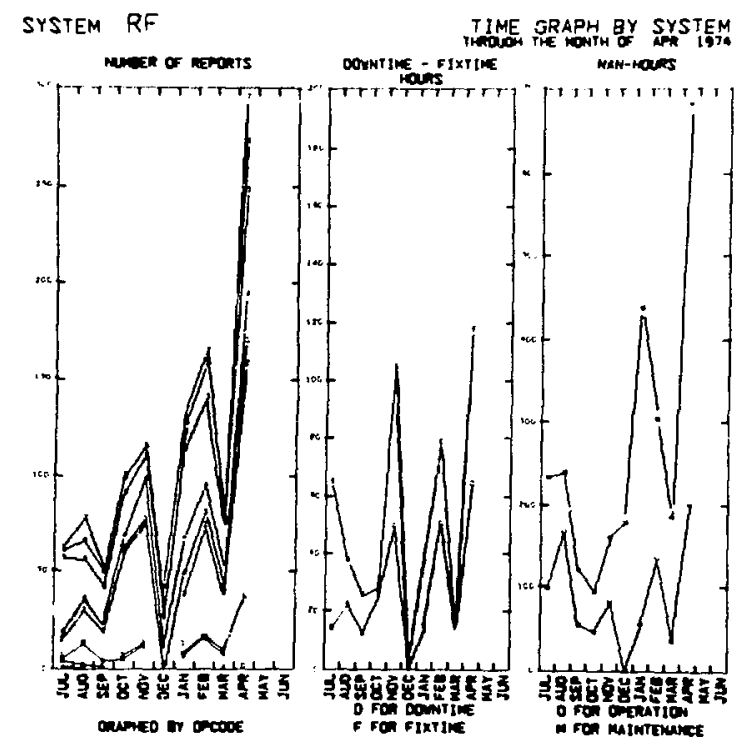

Fig. 11. Running summary of the rf system. (Appendix A.3, B.3.a)

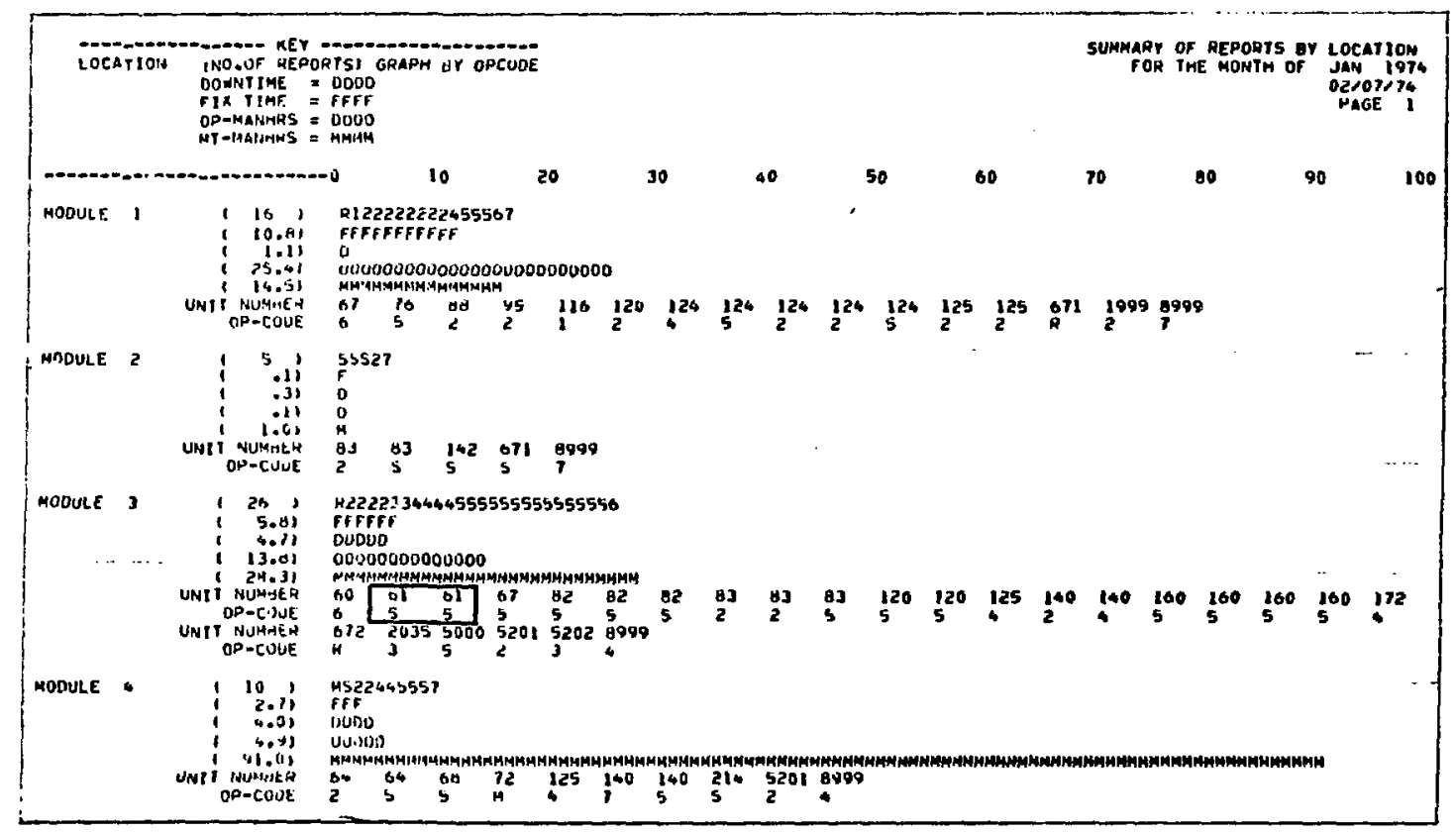

Fig. 12. Summary of reports by location, cross-referenced to unit number by op code. (Appendix A.2) 


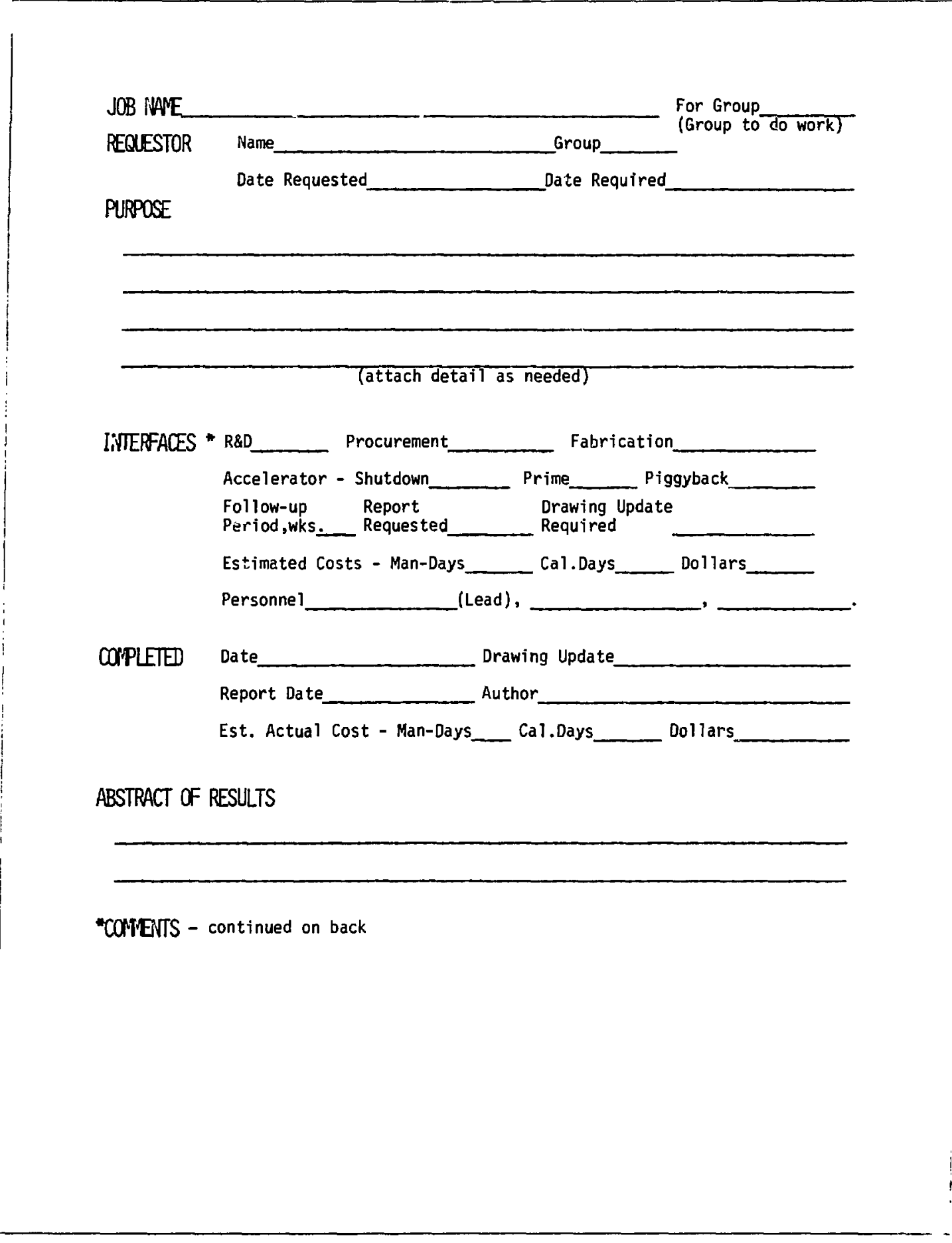

Fig. 13. Work request form. (Appendix A.4) 


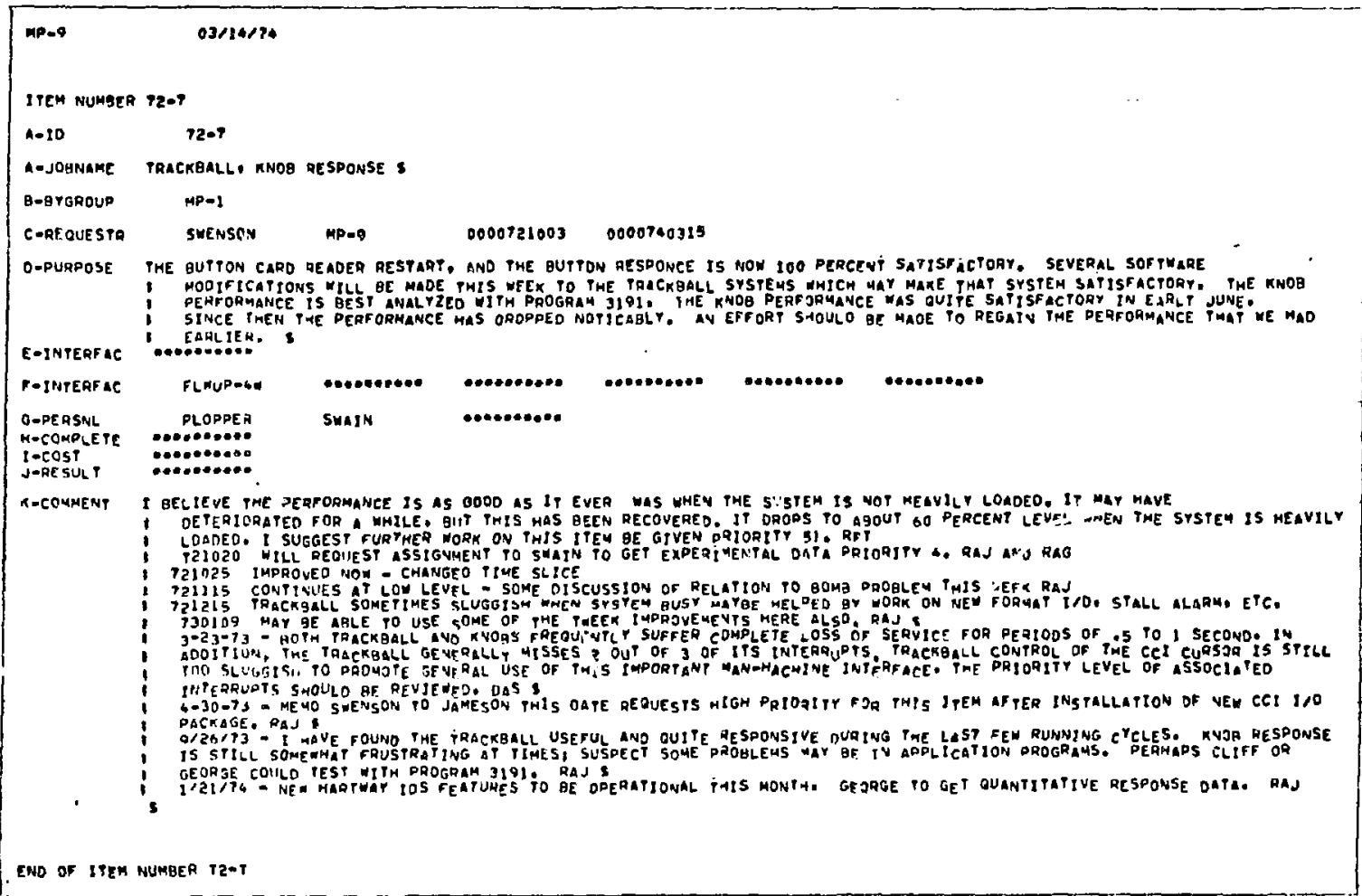

Fig. 14. Example of a typical work request and subsequent actions. (Appendix A.4) 


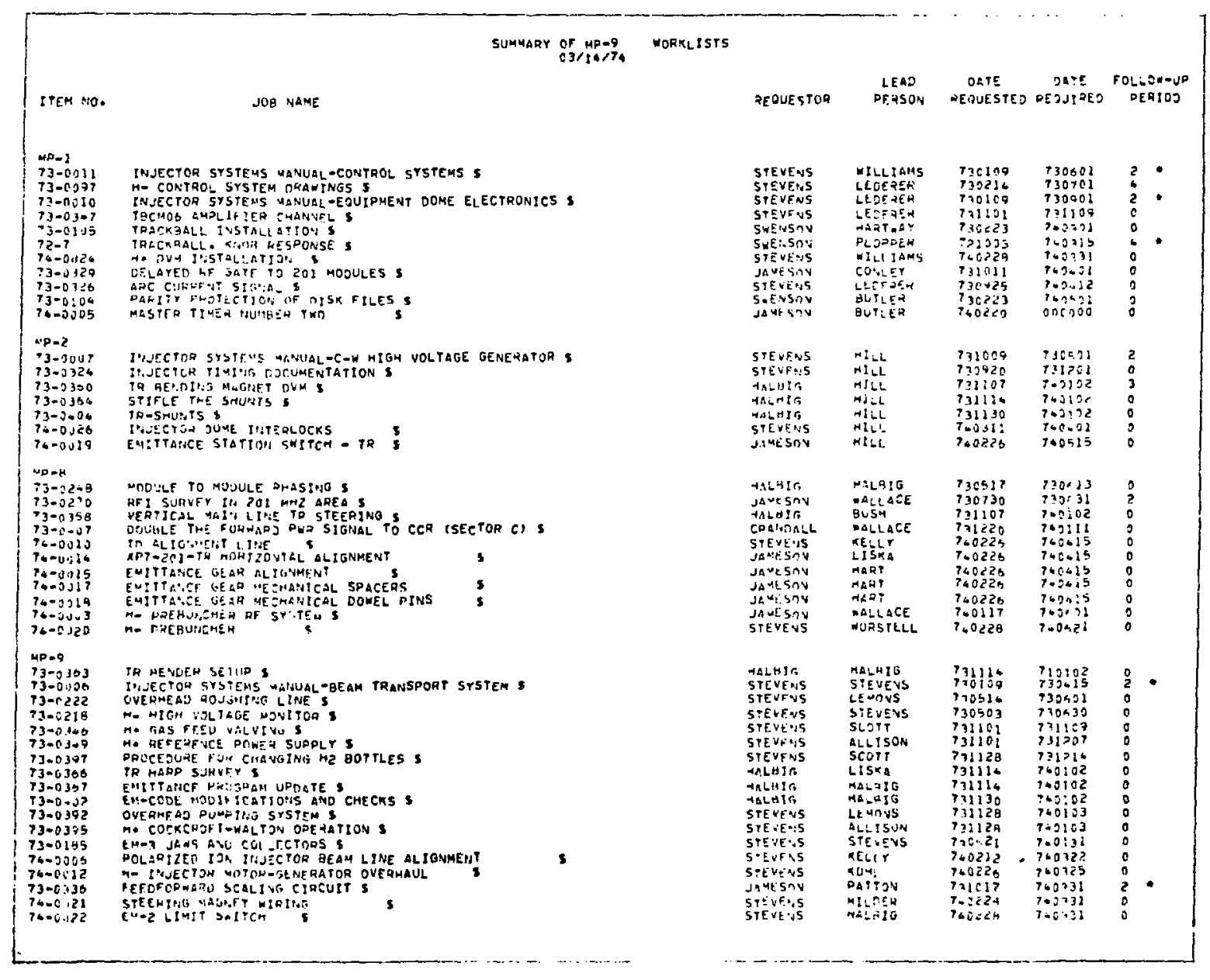

Fig. 15. A summary of work request information supplied weekly to the requesting group; used to coordinate followup action. (Appendix A.A, B.2.b) 


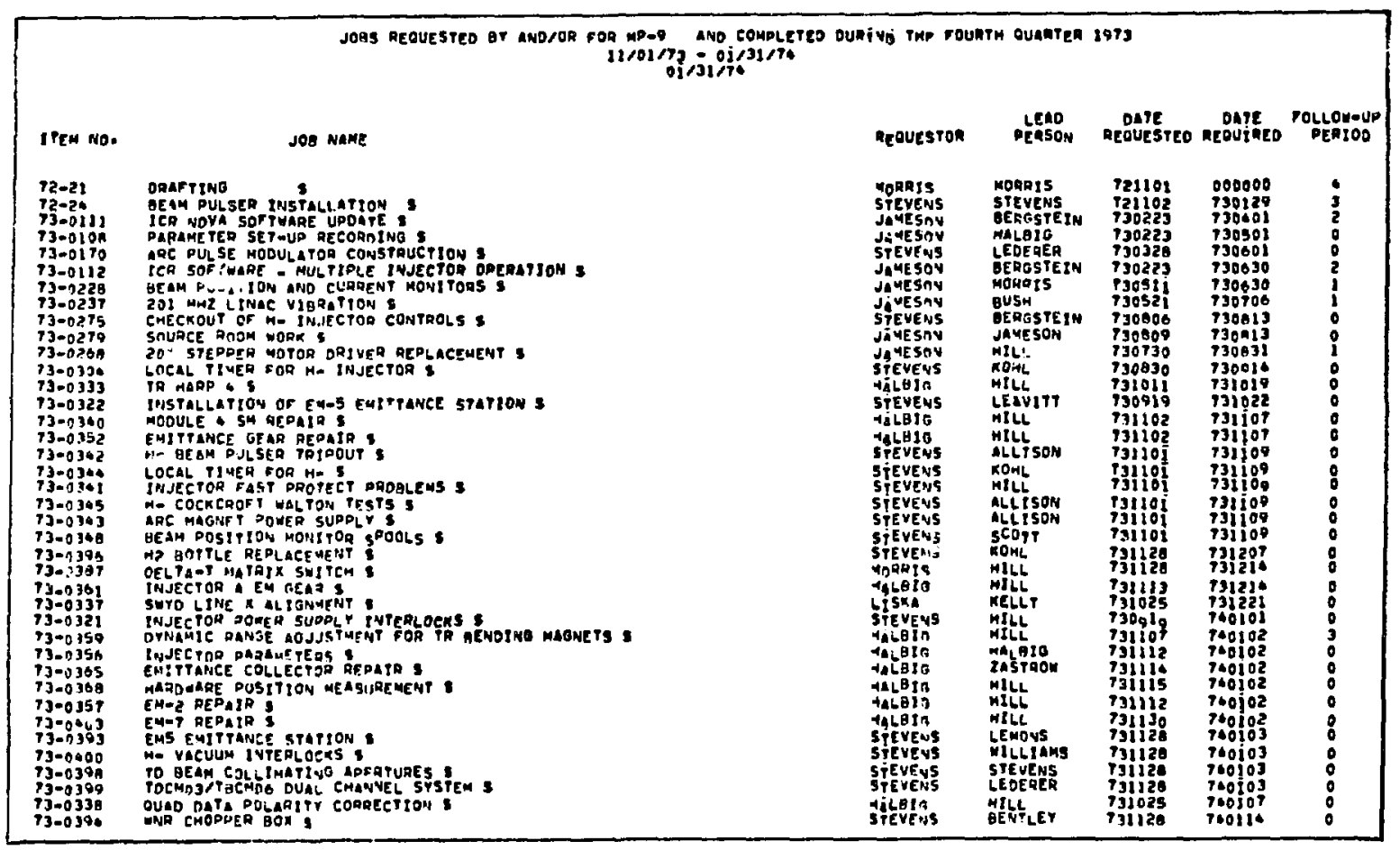

Fig. 16. Quarterly summary of completed work requests. (Appendix A.4) 


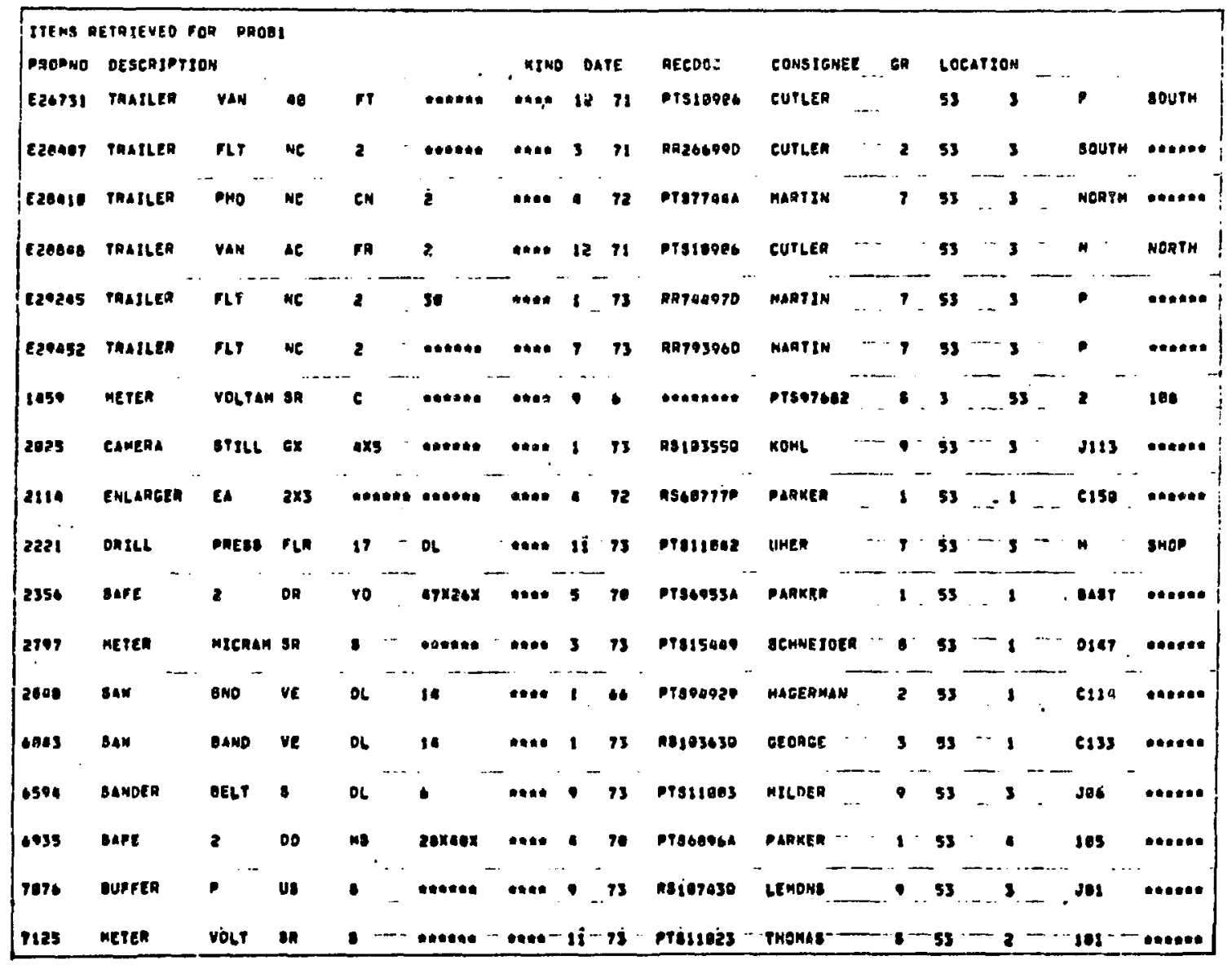

Fig. 17. Format for the capital equipment property control data-base. (Appendix A.5, B.1.b, B.1.C, B.2.C) 


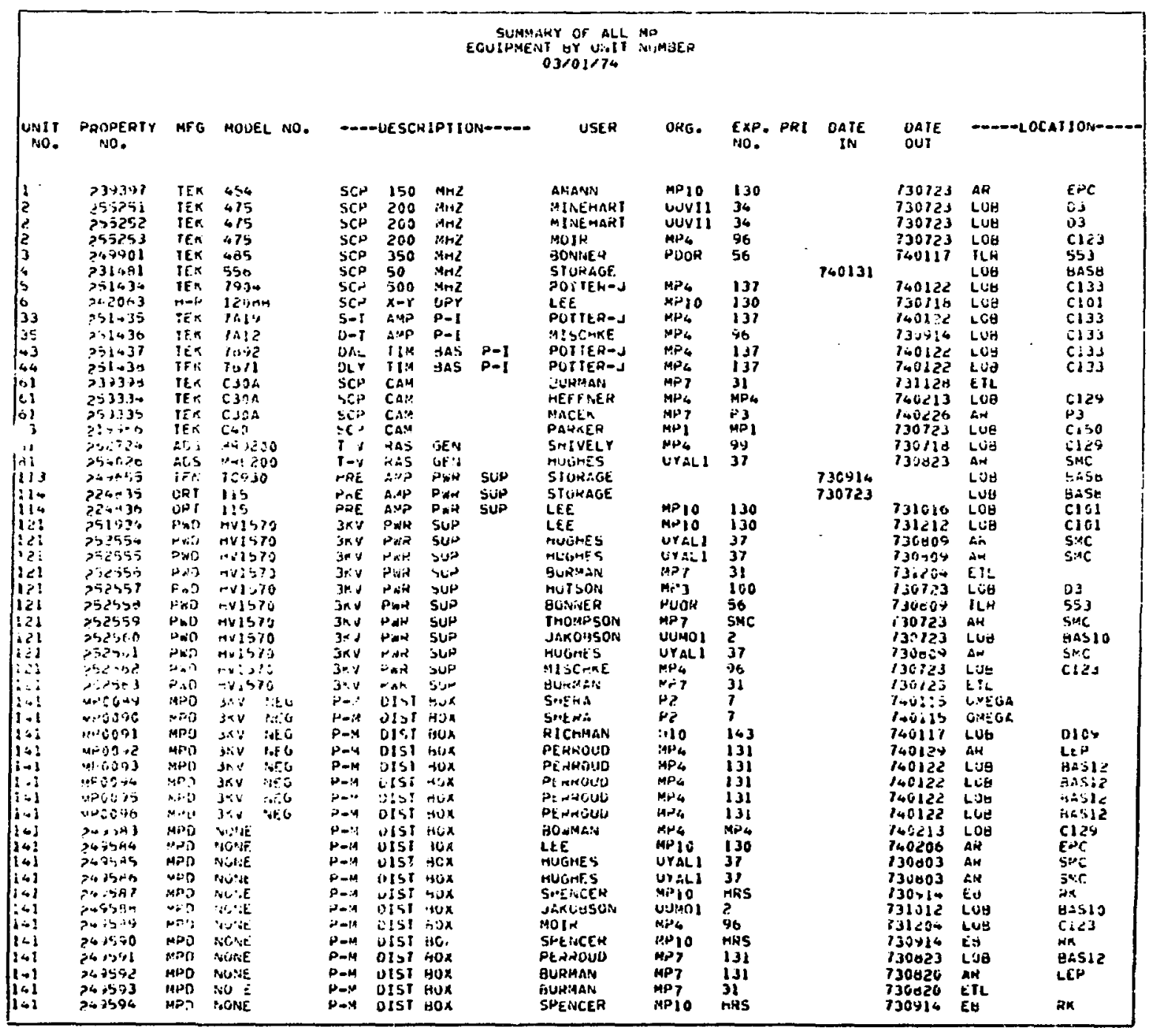

Fig. 18. Summary of pool equipment and allocation information. (Appendix A.6, B.1.d, B.2.d) 


\begin{tabular}{|c|c|c|c|c|c|c|c|c|c|c|c|}
\hline \multirow{3}{*}{ 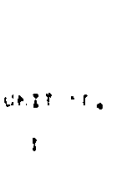 } & \multirow{3}{*}{$p_{i=} F$} & \multirow{3}{*}{$\begin{array}{c}-8 C_{1}+R_{0} \\
.54\end{array}$} & \multicolumn{7}{|c|}{ 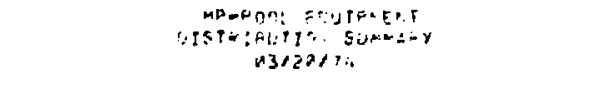 } & \multirow{2}{*}{\multicolumn{2}{|c|}{ cretajaution }} \\
\hline & & & \multicolumn{3}{|c|}{ DESCHFTIDS } & & \multirow{2}{*}{$\begin{array}{c}\text { rePAL } \\
1\end{array}$} & \multirow{2}{*}{$\begin{array}{c}\text { IAUSE } \\
1\end{array}$} & \multirow{2}{*}{$\begin{array}{c}\Delta V A B L \\
O\end{array}$} & & \\
\hline & & & set & $1 \leq: 4$ & $\infty+\infty$ & & & & & $13 i^{4}$ & $!$ \\
\hline$\therefore$ & $=*$ & $\therefore \prime$ & $\dot{s} \leq r$ & $\therefore \cdot$ & $m-1$ & & 3 & 3 & a & $\begin{array}{l}34 \\
96\end{array}$ & $?$ \\
\hline 3 & $\operatorname{tin}$ & $\therefore 5$ & st.i & 350 & $A H \vec{a}$ & & 1 & 1 & 8 & st & $!$ \\
\hline 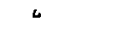 & クレ & $\sin$ & sco & $5 "$ & $\because 47$ & & 1 & $a$ & 1 & & \\
\hline . & $\therefore$ & $\because *:$. & 5 & $5: 5$ & $\cdots i$ & & 1 & 1 & $?$ & $: \$ 7$ & ' \\
\hline . & - & $\therefore \cdots$ & $5+2$ & $x-r$ & $\because 0 y$ & & 1 & 1 & ${ }^{2}$ & $33:$ & $!$ \\
\hline 33 & $\because 2$ & $7: 19$ & $s=1$ & A. 5 & $P-I$ & & 1 & 1 & $A$ & 137 & 1 \\
\hline$\therefore$ & $\therefore$ & $\therefore$ ir & $1-?$ & $A 3$ & $P=1$ & & 1 & 2 & A & só & 1 \\
\hline ' & $\because \cdot$ & $\because r$ & $\therefore t$ & $\because:$ & cis & $P=:$ & 1 & 1 & " & 137 & 1 \\
\hline & $\because:$ & $7 w 71$ & $x r$ & $r: 4$ & 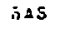 & $P=1$ & 1 & 1 & $a$ & 137 & $:$ \\
\hline$\because$ & $2 t+$ & esies & SEP & cer & & & 3 & 3 & $n$ & $\begin{array}{l}0 . P_{4} \\
23 \\
31\end{array}$ & $\begin{array}{l}1 \\
1 \\
1\end{array}$ \\
\hline$:$ & $\because: a$ & $\therefore$ & $\therefore r$ & $\therefore A$ & & & 1 & 1 & $a$ & 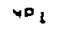 & $i$ \\
\hline$=1$ & $n$ & mert: & $i-y$ & $A A S$ & GES & & $?$ & 2 & $B$ & $\begin{array}{l}37 \\
99\end{array}$ & 1 \\
\hline$: 13$ & $r=$ & $\because 8.4$ & $2+1$ & $A \cdot P$ & 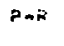 & Siso & 1 & 1 & 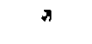 & 145 & 3 \\
\hline$::$ & $r::$ & $:: 4$ & $=\pi$ & $=:$ & $2 n \pi$ & SuP & 2 & $?$ & $a$ & $\begin{array}{l}130 \\
143\end{array}$ & 1 \\
\hline $1: !$ & P.: & $4: 577$ & $3 k v$ & Pand & SUP & & 11 & 12 & B & $\begin{array}{l}\text { swe } \\
2 \\
31 \\
37 \\
50 \\
36 \\
190 \\
130\end{array}$ & $\begin{array}{l}1 \\
1 \\
? \\
3 \\
1 \\
1 \\
1 \\
1\end{array}$ \\
\hline$: \cdots:$ & $\infty \cdot$ & aseir & $P=$ & $015 ?$ & $+n x$ & & 21 & $2 !$ & 3 & $\begin{array}{l}\operatorname{cas}_{2} \\
\cdots 040 \\
2 \\
31 \\
37\end{array}$ & $\begin{array}{l}3 \\
1 \\
1 \\
1 \\
2\end{array}$ \\
\hline
\end{tabular}

Fig. 19. Distribution surmary for pool equipment. (Appendix A.6, B.2.e) 


\begin{tabular}{|c|c|}
\hline ME Y:IEFSH IP LS:I - WDH-LASL & $\begin{array}{l}\text { DATE CF RUR } 0.3 / 20 / 7 \% \\
\text { FAUN DU VEAS:ON }\end{array}$ \\
\hline risen $\quad 1$ & CHEATED J3/20/74 \\
\hline NABIE ARL AIIUMESSS & C1TIZËNSH1P \\
\hline 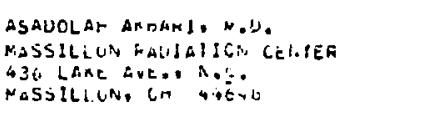 & IRAN \\
\hline 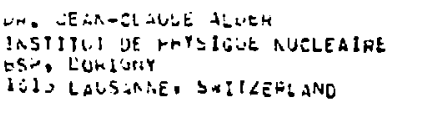 & setss \\
\hline 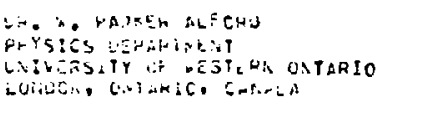 & CLAMDA \\
\hline 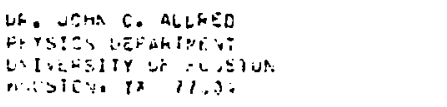 & \\
\hline 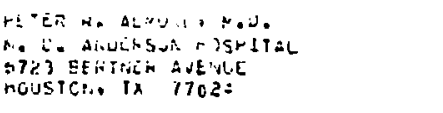 & BRITISH \\
\hline 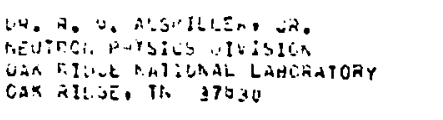 & \\
\hline 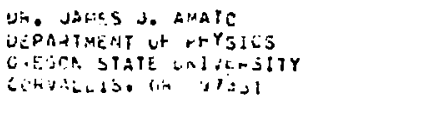 & \\
\hline 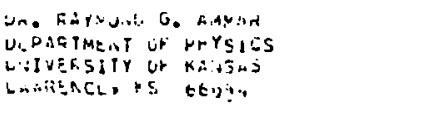 & \\
\hline 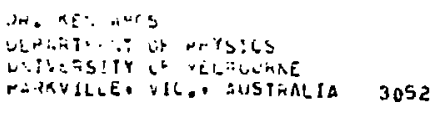 & AUSTRALIAN \\
\hline 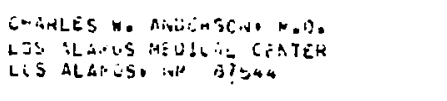 & \\
\hline
\end{tabular}

Fig. 20. Output of a query to the LAMPF Users' data-base for nonLASL users arranged alphabetically, with nationality if not United States citizens. (Appendix A.7, B.2.f)

\begin{tabular}{|c|c|c|}
\hline ENIJCATIONAL & 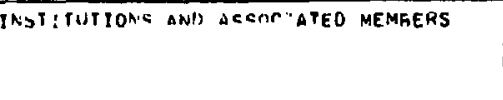 & $\begin{array}{l}\text { PAGE } 16 \\
\text { DARE OF RUN PAG/16/74 } \\
\text { FAUM DR VERSYON } 21 \\
\text { CREATED OC/ } 22 / 74\end{array}$ \\
\hline INST-COSE & INSTITUTE AND MEMEFRSH:P & \\
\hline UV:ค3 & 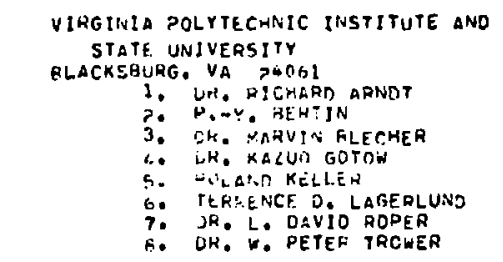 & \\
\hline Unas: & 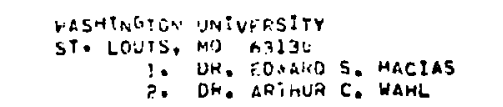 & \\
\hline $4 \times \Delta 53$ & 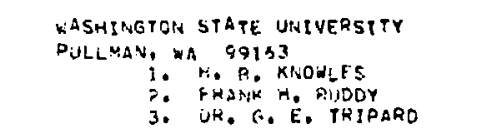 & \\
\hline unres & 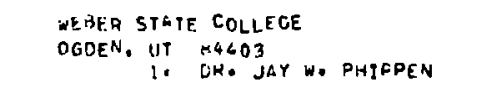 & \\
\hline 4ะ: 42 & 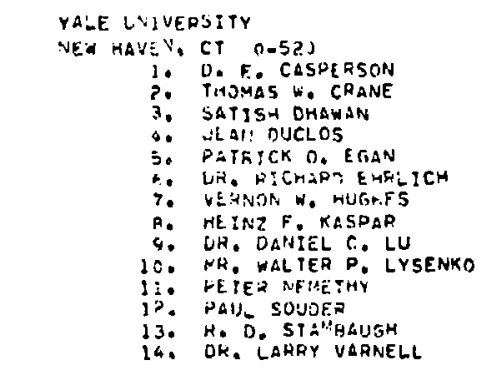 & \\
\hline TOFPF AAE & 399 PERSONISI IN THIS SECTION AND & \\
\hline THE AE ARE & UI INSTITUES IN PMES SECTION. & \\
\hline
\end{tabular}

Fig. 21. A listing of users listed by institution. (Appendix A.7, B.2.g) 


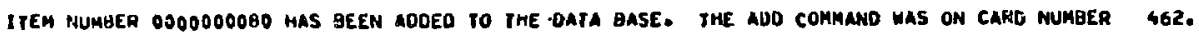

ITEM MUMBER OOOOOOOOBO

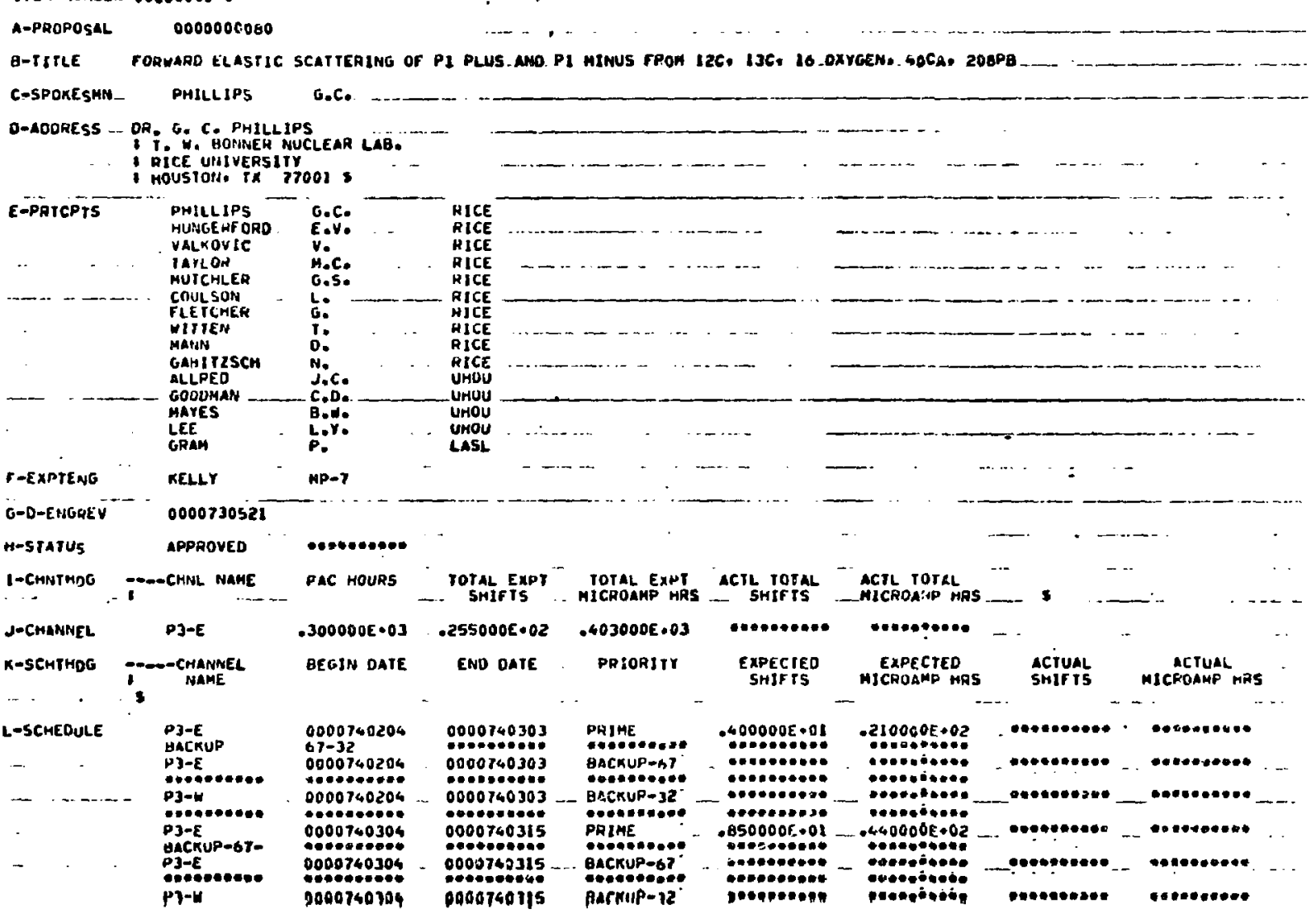

Fig, 22. Typical example of information stored concerning an experiment which has been assigned beam time at LAMPF. (Appendix A.8) 


\begin{tabular}{|c|c|c|c|c|c|c|}
\hline PRCH.NOE. & ? STATUS & CONDITINAS & SPOKESMAN & & PAC HOURS & CHANNEL \\
\hline 1 & PFEFR & NSF $X T=P A C$ & THIMEN & $4 . P$. & $\pi \cdot \pi$ & LEP \\
\hline 2 & APOKNVFD & & JAKOBSDA & $M$. & 150.0 & LEP \\
\hline 3 & APPLAVFD & PAHASITF-R & KINSEY & 3.5. & n. & SMC \\
\hline 4 & AFHEVER & & $\begin{array}{l}\text { IFO } \\
\text { ROLFE }\end{array}$ & $\begin{array}{l}5.5 . \\
R . M .\end{array}$ & 190.0 & HRS \\
\hline 5 & AFPFIVER & & $\begin{array}{l}\text { CHRIEN } \\
\text { PALEVSXY }\end{array}$ & $\mathrm{H}$ & $2 \pi+\pi$ & HRS \\
\hline b & APPHCVEO & & $\begin{array}{l}\text { SUTTER } \\
\text { IG }\end{array}$ & $\begin{array}{l}H, J . \\
G, J .\end{array}$ & 200.0 & HRS \\
\hline $\begin{array}{l}7 \\
9\end{array}$ & $\begin{array}{l}\text { AFPGEIVER } \\
\text { OEFFG }\end{array}$ & $w 1 T H=12$ & PERKINS & R.E. & $6 \% .9$ & $S M C$ \\
\hline 9 & $\triangle B P K$ IIVT & & SABחTTKA & S.E. & $25: 1.9$ & EPICS \\
\hline 10 & APDARVEO & & THIESSFN & $H, A_{\text {, }}$ & $25 \% .0$ & MRS \\
\hline 11 & ADPERVED & $N I I H-101$ & HOE HM & F. & 170.0 & SMC \\
\hline $1 ?$ & APFHTVE? & & PONERS & R.J. & $6 \pi, D$ & SMC \\
\hline $\begin{array}{l}13 \\
14\end{array}$ & AFPETVFD & $W I 1 H / 70$ & $S \in T$ th & $k . k$. & 10.7 .0 & EPICS \\
\hline $\begin{array}{l}10 \\
15\end{array}$ & $\begin{array}{l}\text { AFPRTIVED } \\
\text { AWPH TYF S }\end{array}$ & & $\begin{array}{l}\text { THTESSEN } \\
\text { TANAKA }\end{array}$ & $\begin{array}{l}\mathrm{H}_{0} \mathrm{~A}^{-} \\
\mathrm{N} .\end{array}$ & $\begin{array}{l}250.0 \\
20.0 .0\end{array}$ & $\begin{array}{l}\text { FPICS } \\
\text { MRS }\end{array}$ \\
\hline 16 & APPLCVER & & MEYER & S.1. & 10.0 & $\operatorname{sic}$ \\
\hline 17 & APPMONEO & & $\begin{array}{l}\text { MURR ISON } \\
\text { LE IDMAN }\end{array}$ & G.C. & 230.4 & EPICS \\
\hline 18 & APPQDVED & & ZETOMAN & A. & 259.0 & EPICS \\
\hline $\begin{array}{l}19 \\
20\end{array}$ & $\begin{array}{l}\text { AFPRTVED } \\
\text { ITHDRAWN }\end{array}$ & & LIERER & $\Delta$. & 75.9 & LEP-AIOMED \\
\hline 21 & RE JFETTD & & & & & \\
\hline $\begin{array}{l}22 \\
23\end{array}$ & $\begin{array}{l}\text { APPFOVFO } \\
\text { APPFOVFO }\end{array}$ & & $\begin{array}{l}\text { GEMMELL } \\
\text { PAHINES }\end{array}$ & $\begin{array}{l}\text { D.S. } \\
\text { P. }\end{array}$ & $\begin{array}{l}153.0 \\
250.0\end{array}$ & $\begin{array}{l}\text { EPICS } \\
\text { EPICS }\end{array}$ \\
\hline 24 & DEFER & $F F A S=5 T(1) Y$ & & & & \\
\hline 25 & APPAOVFA & & $13\{14 M A N$ & R.L. & 200.11 & LFP \\
\hline 26 & APBISVET & & VFFSFR & L. & 100.0 & $E P A$ \\
\hline 27 & ADPANVEO & PHASE=1 & ILLARD & M.P. & $\sin 00$ & EPE \\
\hline 28 & $\begin{array}{l}\text { APPNCIVOD } \\
\text { APDACIVED }\end{array}$ & & YAVIN & A.I. & $\ln 10.0$ & LEP \\
\hline $\begin{array}{l}29 \\
30\end{array}$ & $\begin{array}{l}\text { APPRTIVED } \\
\text { HEJETTEO }\end{array}$ & nTtros 50 & GOTOW & $k_{\text {, }}$ & 190.0 & LFP \\
\hline 31 & APSWOVFD & & NEMETHY & P. & BOR.A & NE UTR IND=A \\
\hline 32 & $\triangle P P R O V E D$ & & $\begin{array}{l}\text { MCFAFL ANE } \\
\text { MACFK }\end{array}$ & & 380.0 & $\mathrm{P}_{3}-W$ \\
\hline 33 & APDF DVED & & HWANCO & c. & 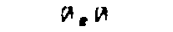 & EPB \\
\hline 34 & APPHITVED & & M INE HAST & F. & 223.9 & $P 3-E$ \\
\hline $\begin{array}{l}35 \\
36\end{array}$ & $\begin{array}{l}\text { APPROVEB } \\
\text { RESUBMIT }\end{array}$ & & 7 Iock & $K, O, H$. & 140.11 & LEP \\
\hline 37 & APFENVED & & MUGHES & $0.4 \%$ & 549.9 & $S M C=S$ \\
\hline $\begin{array}{l}3 A \\
30\end{array}$ & $\begin{array}{l}\text { FEAS-STYDT } \\
\text { SPBIOUVEO }\end{array}$ & & $\begin{array}{l}\text { RF INES } \\
\text { MACEK }\end{array}$ & F.J. & 250.0 & $\begin{array}{l}\text { NEUTRIND=A } \\
\text { EPICS }\end{array}$ \\
\hline पन & IA ITHCRAWN & & & & & \\
\hline$\Delta !$ & DEFEP & & & & & \\
\hline 42 & RESUAMIT & & & $1, W$, & 250,0 & \\
\hline $\begin{array}{l}43 \\
44\end{array}$ & $\begin{array}{l}\text { APPPCVED } \\
\text { APPR DVEI) }\end{array}$ & & $\begin{array}{l}\text { SWFNSON } \\
\text { CARLSON }\end{array}$ & $D . E$ & 3 in. & $\begin{array}{l}\text { EPICS } \\
\text { GIOMEO }\end{array}$ \\
\hline 45 & APPHCIVFD & & $\begin{array}{l}\text { DUDZIAK } \\
\text { GHEEN } \\
\text { GIORGI }\end{array}$ & $\begin{array}{l}0.5 . \\
0.2 . \\
0.1 .\end{array}$ & 0.0 & $M L I N=L$ INEA \\
\hline $\begin{array}{l}46 \\
47\end{array}$ & $\begin{array}{l}\text { APPPNVED } \\
\text { ALPROVFD }\end{array}$ & & $\begin{array}{l}\text { FFTERSON } \\
\text { PETERSON }\end{array}$ & $\begin{array}{l}R .3 . \\
R .3 .\end{array}$ & $\begin{array}{l}399.0 \\
190.0\end{array}$ & $\begin{array}{l}\text { FPICS } \\
\text { EPIES }\end{array}$ \\
\hline $\begin{array}{l}48 \\
40\end{array}$ & $\begin{array}{l}\text { UEFEN } \\
\text { APPNCUED }\end{array}$ & & PETERSON & $R, J$ & 75.0 & HR 5 \\
\hline
\end{tabular}

Fig. 23. A summary of experimental proposal status arranged numerically by proposal number. (Appendix A.8, B.2.h) 
paFticipat? TR.

$\triangle S$ TF $R \angle I I R / 7 L$

part $A$

LAST NAHE INITIALS THSTITUTE EXPERIMEPT INVOLVFMENT

SIETEL R.T CHN

SIEMSSEN F.". WPADCFPDIE

5IMMONS I.F. IASL SO

SIMON H.T. linY 110

SHITH H.H. NRL 113

SOBEI. IIII IUR

SCADTTKA S.t. UVA

SPFMTEK J.E. LASL

SPEMTNRTE J.

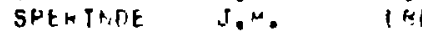

SPFERY W.T.

SPRACIIE J.A.

SPOINI;FH H.

STARNFW J.N

STFARNS K.L.

STFFFEN

STE IN

STF INFF RT

STE 72

STFUTAS

STRANT,

STIJPI.

SUNIFK

SUTTEH

SnALLON

5 WF NON

TANAKA

TAYLCE

TAYLOR

H. . .

N.

$t . P$

A. 1.

$\therefore$

i.

i. 4.

J.,

H.J.

F.

$1 . N$

$n$.

$N$

THICSSF

. $C$.

$\omega .4$.

THOMASON

THOMPSOA

PHOMPSTN

THROOP

TPJPAKC

TFUDFL

TKLIMP

TIAKEVICH

UCLA

$\therefore, \mathrm{li}$

A. C.

P.A.

r. . . .

$p$.

M.

A.

SIFAFIS

$J, F$.

VALKDVJC

VARGHESE

VASSAP

$v$.

$P$

TAKAKA

$L \cdot R$.

VEESER

VIGOCR

VINCENT

VOGE L

WADDELL

- AOL INGEF

5.

J.

$P$.

$C . N$.

C. ASII

NAI

MIJNTCH

L ASL

VASSAQ

PIROUE

LASL

ANL

LHL

I A.SL

IICLA

nRSU

116:LA

ANL

UCHI

OWSLI

LASL

COL-SCTLAH

RICF

I. ASI

IVA

CMLI

LASL

UกA

bSU

IIUK

I $45 \mathrm{~L}$

UC, 1

R.L.

$A+1$

PICF.

Uigh

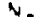

1. ASL

II I I

NASA/LEWIS

CAl.TEC

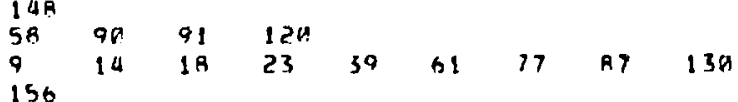

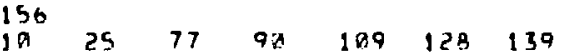

3

151

113

an 160

175

6

$100 \quad 117$

119

74

27

117

$13 A \quad 15 B$

117

5

b 15

$43 \quad 139 \quad 139 \quad 156$

$\begin{array}{lllll}14 & 15 & 59 & 129 & 139\end{array}$

79

$111 \quad 14 \quad 18 \quad 25 \quad 39$ b1 $74 \quad 77$

$132133 \quad 136 \quad 156$

139

$89 \quad 153 \quad 159$

132161

138

151

5 it

19

15

142

79 Aด B1 B3 B4

138

5

26

117

87

$11 \quad 12 \quad 101166 \quad 173$

2773

Fig. 24. A listing of users, showing the experiments in which they are participants. (Appendix A.8, B.2.i) 
APPENDIX A

DATA BASE DEFINITIONS

Fig. A.?. Definition of the Background data-base for acceleracor equipment as specified in the Generalized Information Retrieval-LASL System (GIRLS) data management system. Information upon which retrievals will be keyed is stored in search fields. Other material is stored in text fields. Refer to Figs. 1-2.

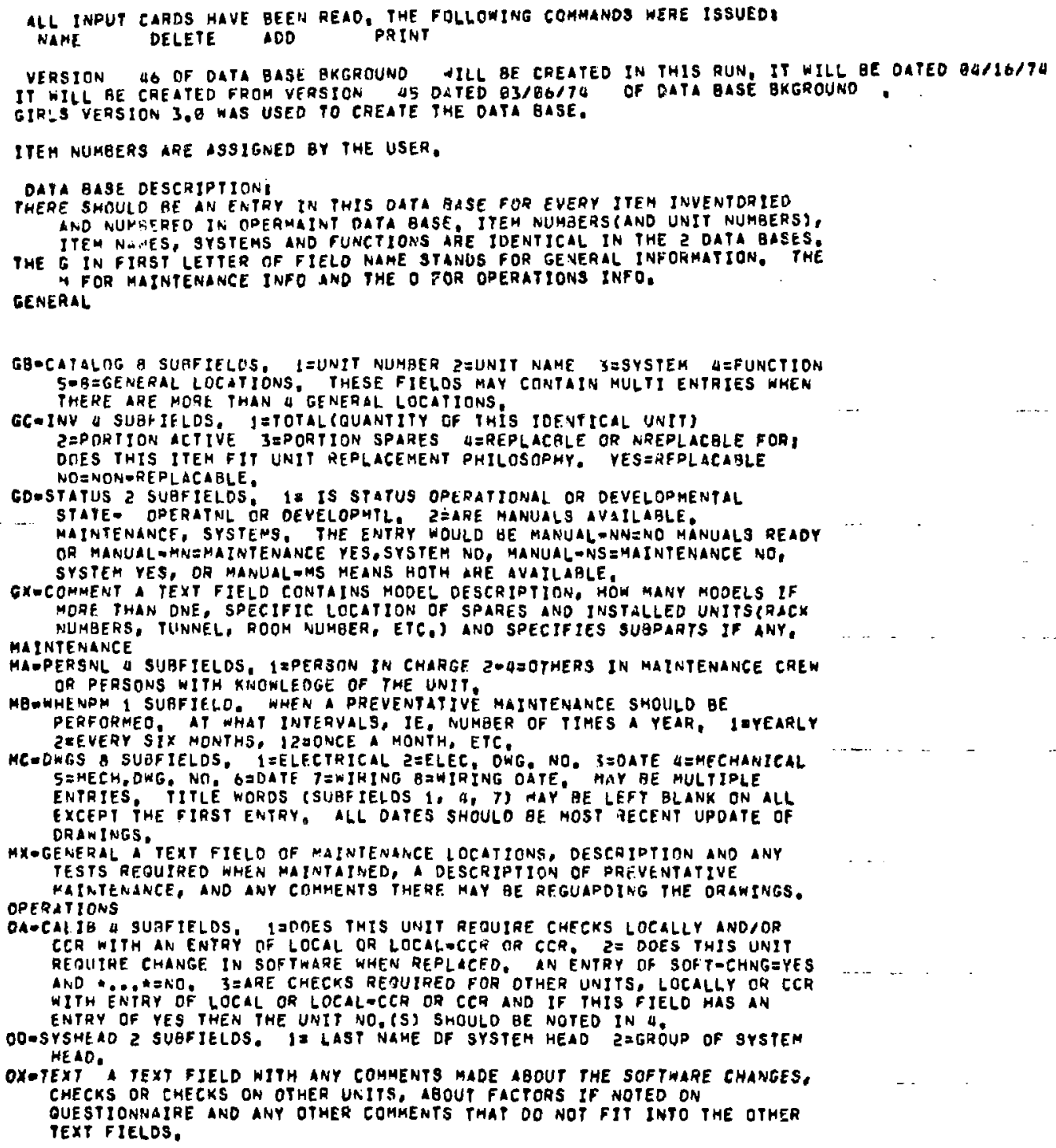

ITEM NUMEERS ARE ASSIGNED BY THE USER.

DATA BASE DESCRIPTIONI

PHERE SHOULO BE AN ENTRY TN THIS OATA AASE FOR EVERY ITEH INUENTORIED AND NUMSEREO IN OPERMAINT DATA BASE, ITEH NUMBERS (AND UNIT NUMBERS), ITE NG NES, SYSTEMS AND FUNCTIONS ARE IDENTICAL IN THE 2 DATA BASES.

THE G IN FIRST LETTER OF FIELO NAME STANUS FOR GENERAL INFORMATION. THE - FOR MAINTENANCE INFO AND THE O FOR OPERATIONS INFO. GENERAL

GB-CATALOG G SUAFIELOS, LIUNIT NUMBER ZEUNIT NAME SESYSTEM $4=F U N C T I O N$ 5-B=GENERAL LOCATIONS, THESE FIELOS MAY CONTAIN MULTI ENTRIES WKEN PHERE ARE MDQE THAN 4 GENERAL LOCATIONS.

GC-INV O SUBFIFLOS. JOTOTAL (GUANTITY GF THIS IOENFICAL UNIT) $2=P O R T I O N$ ACTIVE $3=P O R T I O N$ SPARES $4=R E P L A C B L E$ OR NREPLACBLE FOR, DOES THIS ITEM FIT UNIT REPLACEMENT PHILOSOPKY, YES=REPLACAOLE NO $=$ NON-REPLACABLE,

CDNSTATUS 2 SUEFIELDS, 1 IS IS SPATUS OPERATIONAL OR DEVELOPMENTAL STATE- OPERATNL OR OEVELOPMTL. ZEARE MANUALS AVAILABLE. MAINIENANCE, SYSTEMS, THE ENTRY WOULD BE MANUAL-NNENO MANUALS REAOY OR MANUAL-HMEMAINTENANCE YES,SYSTEM NO, MANUAL-NS=MAINTENANCE NO, SYSTEM YES, OR MANUAL-MS MEANS HOTH LHE AVAILABLE,

GX-COMMENT TEXT FIELD CONTAINS MODEL DESCRIPTION. HOW MANY MODELS IF MOAF THAN DNE, SPECIFIC LOCATION OF SPARES ANO INSTALLED UNIPSLRACX NUMBERS, TUNNEL, ROOM NUMBER, ETC,) ANO SPECIFIES SUGPARTS IF ANY, MAINTENANCE

MA-PERSNL I SUBFIELOS, IIPERSON IN CMARGE 2-4EOTHERS IN MAINTENANGE CREW OR PERSONS WITH KNOWLEDGE OF THE UNIT.

MBNWHENPM I SUBFIELO. WHEN A PREVENTATIVE MAINTENANEE SHOULD BE PERFORMEO, AT WHAT INTERYALS, IE, NUMAER OF TIMES A YEAR, IOYEARLY ZEEVERY SIX MONTHS, I2 2 ONCE A MONTH. ETC.

MCODWGS SUBFIELDS, $1=E L E C T R I C A L ~ 2=E L E C$, OWG. NO. $3=$ OATE $\angle=M E C H A N I C A L$ 5:MEEH, OWG. NO, GJDATE 7=WIHING B=WIRING OATE. MAY BE MULTIPLE ENTRIES, TITLE WORDS (SUBFIELDS 1, a, 7) MAY GE LEFT BLANK ON ALL EXCEPI THE FIRST ENTRY, ALL DATES SHOULO BE MOST TECENT UPOATE OF DRAWINGS.

MXOGENERAL A TEXT FIELD OF MAINTENANCE LOCATIONS, DESCATDTION AND ANY TESTS REOUIRED WHEN MAINTAINED, A OESCRIPTION OF PREVENTATIVE maIATENANCE, and ANY COMMENTS THERE MAY BE RFGUAPDING THE ORAWINGS. OPERATIONS

OA-CALIB 4 SUAFIELOS, IDDOES TMIS UNIT REOUIRE CHECKS LOCALLY ANDIOR CCR WITH AN ENTRY OF LOCAL OR LOCAL-CCF OR CCR. $Z=$ DOES THIS UNIT REOIITE CHANGE. IN SOFTHARE WHEN REPLACFD. IN ENTRY OF SOFT-CHNGEYES $A N D$...*A=NO, $3=A R E$ CHECKS RETUIREO FOR OTHER UNITS, LOCALLY OR TCR WITH ENTRY OF LOCAL OR LOCAL-CCR OR CEA AND IF THIS FIELO HAS AN ENTRY OF YES THEN THE UNTT NO, (S) SHOULO BE NOTED IN 4.

OOASYSHEAD 2 SUEFIELDS. I LAST NAME DF SYSTEH HEAD $2=$ GROUP OF SYSTEM HEAO.

OX-TEXT A TEXT EJELD WITH ANY COMMENTS MADE ABOUT THE SOFTMARE CHANGES, CHECKS OR EHECKS ON OTHER UNITS, ABOUT FACTORS IF NOTED ON QUESTIONNAIRE ANO ANY OTHER COMMENTS THAP DO NOT FIT INTO THE OTHER TEXT FIELOS.

continued 


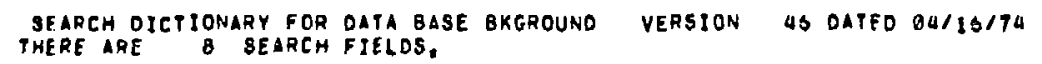

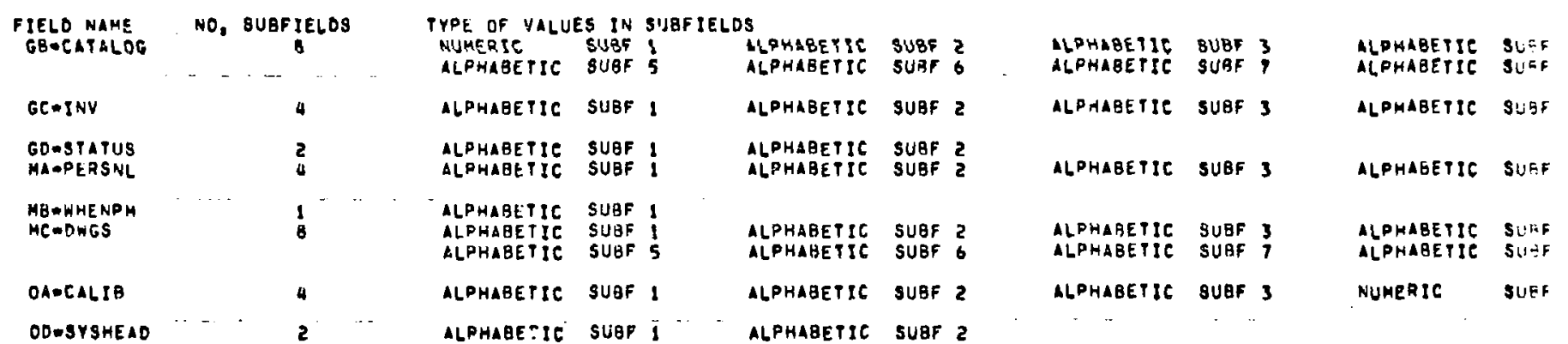

\begin{abstract}
TEXT OICTIONAPY FOR OATA BASE BKGROUNO VERSION 46 DATEO O4/16/74
THERE ARE 3 TEXTFIELLS
\end{abstract}

\title{
FIELO NAME
}

GX-COMMENT

$M X$ G GENER
OX TEXT 


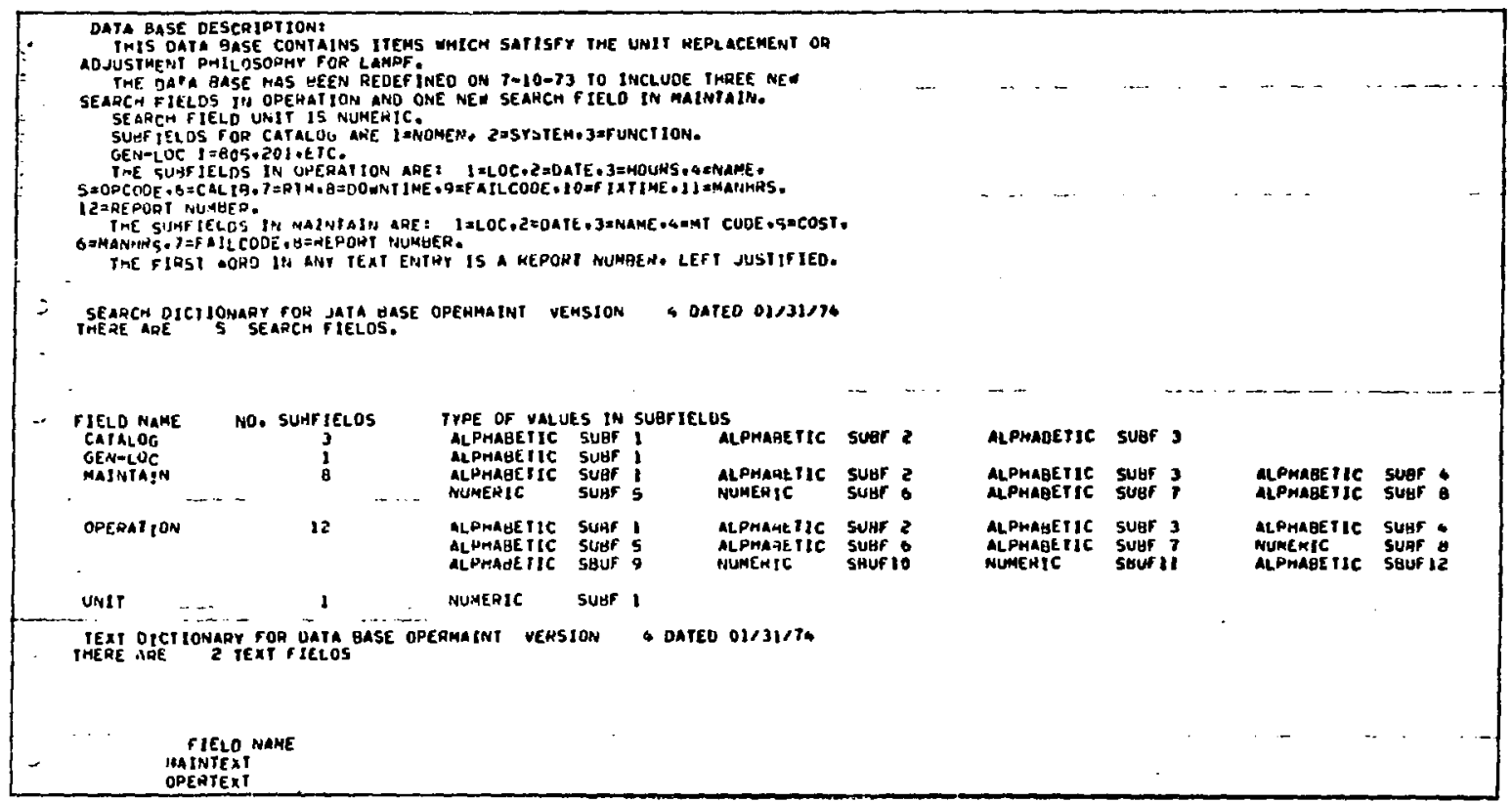

Fig. A.2. Definition of the data-base OPERMAINT for recording the operating history of equipment. Search fields may have up to 20 subfields of either alphabetic or numeric type. Refer to Figs. 5-8 and 12 for examples of ret:-ievals from this data-base. Appendixes B.1.a, B.2.a:, and B.3.b. show input/output progranis associated with this data-base. 


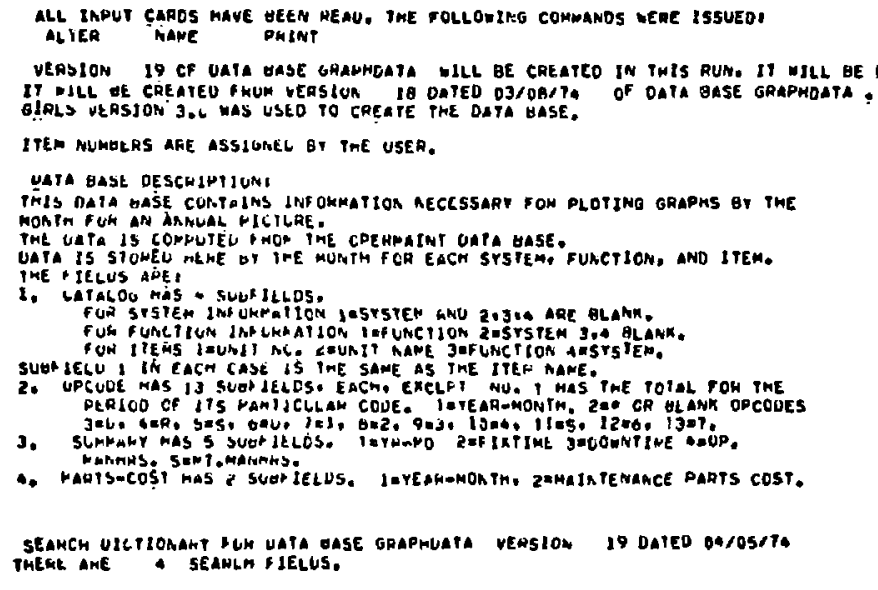

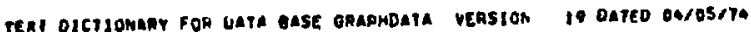
TEERE AKE TERT PIELUS

Fig. A.3. Definition of data-base GRAPHDATA, in which information retrieved from data-base OPERMAlilT is stored for plotting summaries in graphical form as shown in Figs. 9-11. See also Appendix B.3.a. 


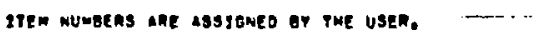

oura ane oeschiersom

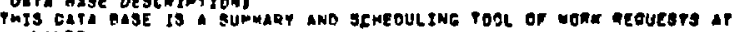

Lamer.

ATELDS ADF

Anto (1 SUOF TELOS - ITEm HAME

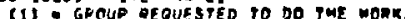

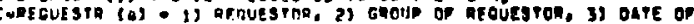

KEQUEST, QS CATE TO AE CE VOLETED.

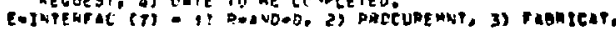

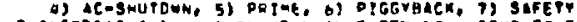

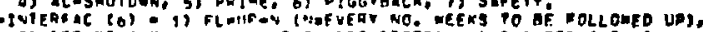

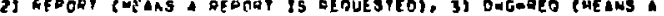

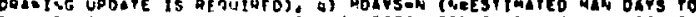

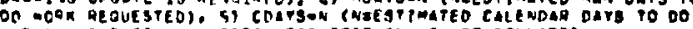

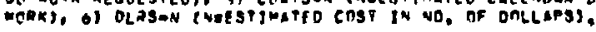

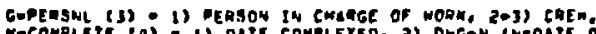

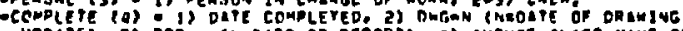

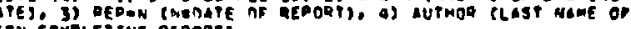

cosi $(5)$ is

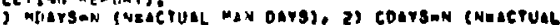

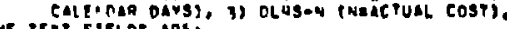

Fietos ante

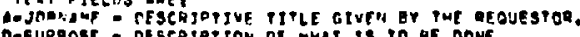

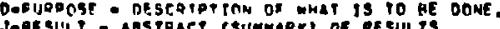

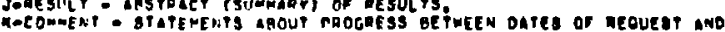
COMLETIOA.

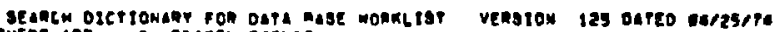
intue ine seanew Fielos.

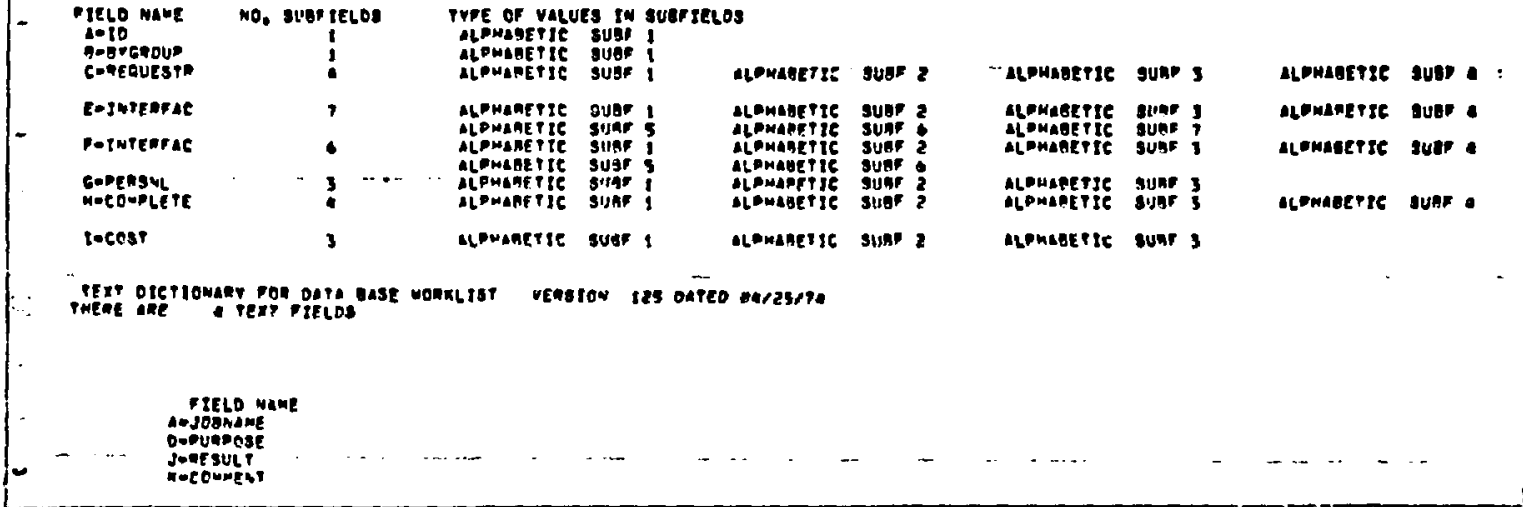

Fig. A.4. Description of the data-base "Work1ist" for work requests. See Figs. 13-16 and Appendix B.2.b. 


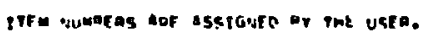

nats anse oeseciotions

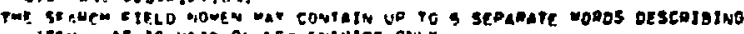

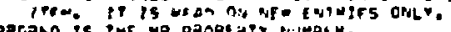

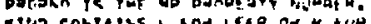

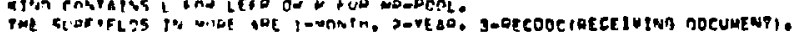

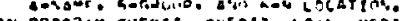

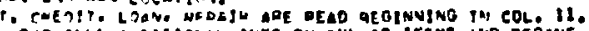

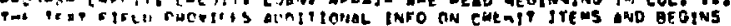

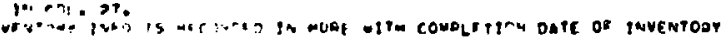

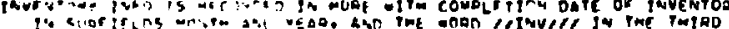

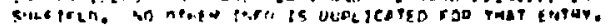

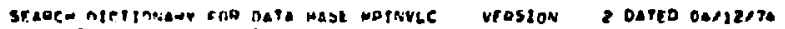

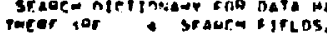

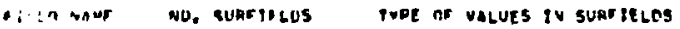

agiri alPmanetic suar I

alomafite suar?

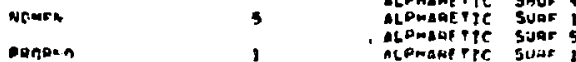

alomagrtic suar

alomatite suaf.

alamegrtic suw

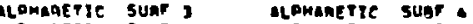

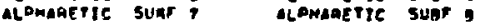

eqnoen

I

ncenante juar?

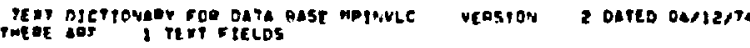

tiflo ean

tet

Fig. A.5. Definition of the data-base for the control of capital-equinment property. See Fig. 17 and Appendixes B.l.b, B.l.C, and B.2.C. 
ITEM NUWEERS AAE ASSIGNEO BY THE USER.

DATA GASE DESTRipTIOA:

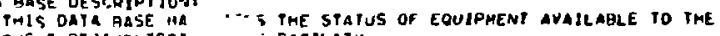

VANIOUS EAPEAIMESTEPS Ö. FACLLITY

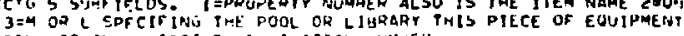

3=4 Jo

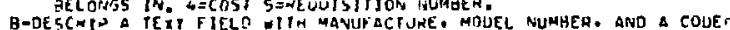

OESCNINTION OF TIEM.

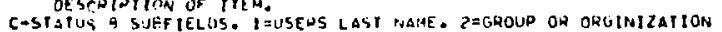

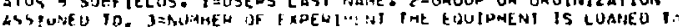

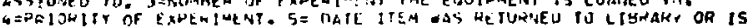

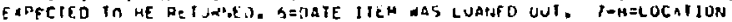

DF FAIIPAET

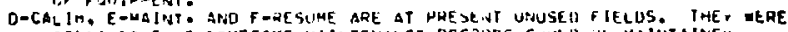

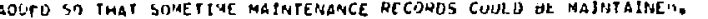

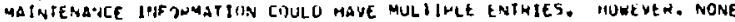

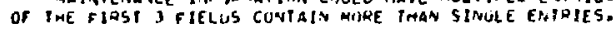

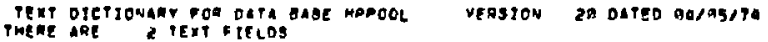

- JEL D name

o-oejcage

paresume

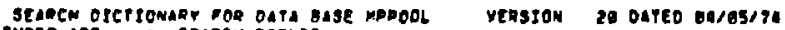

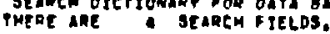

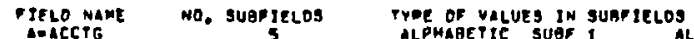

5 ALPHABETIC SUGF

cestapus

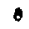

ALPHAEETIC SUOF

ALPMAGETIC OUPF 3

numerte

ALPHARETIC SUBF 3
aLPMAGETIC SUGF

OCEAL IB
EANATT

aLOHARETIC SUB⿱

ALPHAAETJE SURF

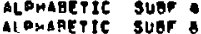

3

IEPAAETIC SUAF

ALPHAEETIC SURF ?

ALPHABETIC SUAF 3

Fig. A.6. Data-base definition for maintaining allocation status of equipment in the experimental apparatus pool. See Figs. 18 and 19 and Appendixes B.1.d, B.2.d, and B.2.e. 


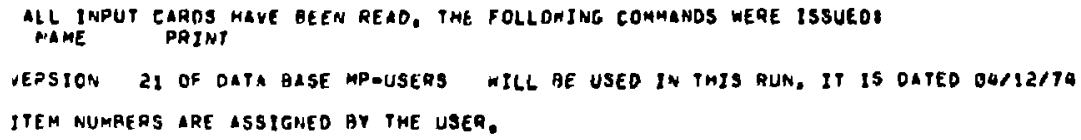

$\begin{array}{ll}\text { AITFMENAME } & \text { LLPMABETIE SUBF I } \\ \text { CITIZEN } & 1\end{array}$

NO. SUAF IELDS
ALPMABETIC SUBF !
ALPHARETIE SUEF ? ALPHARETIC SUBF ALFMARETIC SUBF ALPHARETIC SUBF alPHARETIC SUaF ALPHABETIC SUgF 1

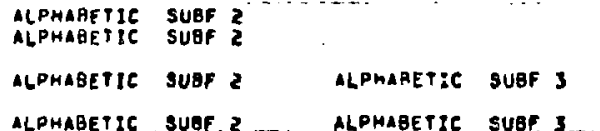

ALPHABETIC SUAF.
ALPHABETIC SUBF. $3 .$.

TEXT DICTIONARY FOR OATA GASE MPAUSEAS VEASION 21 OATED OAIIZTIA

THERE ARE S TEXT FIFLOS

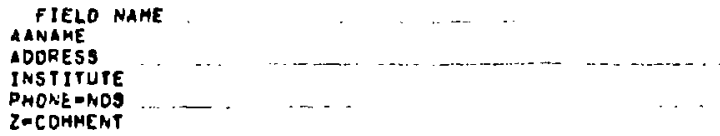

Fig. A.7. Definition of data-base for information concerning users of LAMPF. See also Figs. 20-21 and Appendixes B.2.f and B.2.g. 
DATA BASE OESCRTPTION:

THIS DATA GASE HAS BEEN DESIGNEU TO LIST ALL RUOHS AT LAMPF, TO UEF INE THEIF TYPE. AHD TO RECORO CURKEHT OCCUPANIS ANU GHUUP, PHOUE VU:HEPS. PNESENT USE, AND COMHENTS WMERE APPKOHAIATE.

THE SEARCH FIELDS ARE:

1. ROOM, 2 SURFIELOS. HLDG aNO ROOM NUMBE'R. TRallers mar have no ENTRY IN SECONO SUBF IELD. SINGLE ENTHY ALWAYS.

2. TYPE. I SUPF IEL.L. ASSUMEO SIMGLE ENTRY. MAY INCLUUE IMPL ED IRFORMATION ABOUT SIZE (2-OFFICE).

3. USE. 1-SUHF IELO. ASSUMEU SINGLE ENTRY TO EXPLAIN CURKENT USE. PAR! Í́TULARLY FOR LAH OR SPECIAL SPACE.

4. OCCUPANT, 2 SUHFIELUS. LAST HAME AINU GROUP. MULTIPLE ENT:IES EXPECTFD. IF NO OCCUPANT, RESPONSIALF GROUP IN SUBFIELr 2.

S. PHONE. I SUHF IELD. HAY MAVE MULTIPLE EINTRIES KEPT IN COROTET ORDER OF OCCUPANTS.

A SINGLE TEXT FIELO--TEXT--CONTAINS OKIEF AOOITIONAL OESCRIPTIONS KEPT IN CORRECT ORDER IF THEY APPLY TO SPECIFIC OCCUPANTS. CAUTIOY MUST WE USEO IN DELETING OH MOUIFYING INFORMATIUN.

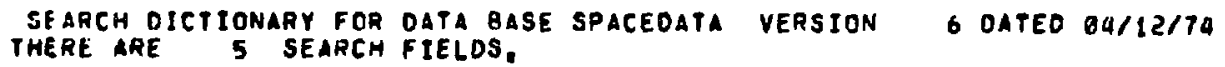

F IELD NAME OCCLPANT PHONE RCOM TYPE

use

NO. SUBFIELOS
2
$?$
2
1
1

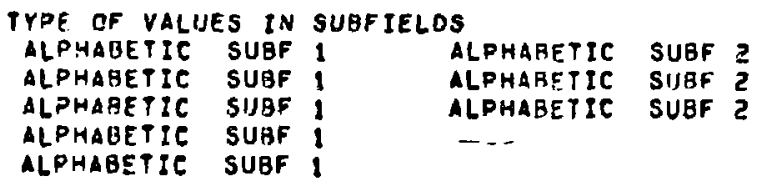

TEXT DICTIONARY FOR DATA BASE SPACEDATA VERSION THERE ARE I PEXT FIELOS

F IELD NAME

ROREMIRKS

Fig. A.9. Data-base "Spacedata" is used to coordinate the allocation of office and laboratory space. See Appendix B.4.a. 
APPENDIX $B$

LASL Identification: LP-0356

PROGRAM LISTINGS

B.1. Pre-Processor Programs. These programs are translators which take information from the user input format and prepare it for use by the GIRLS data-management program.

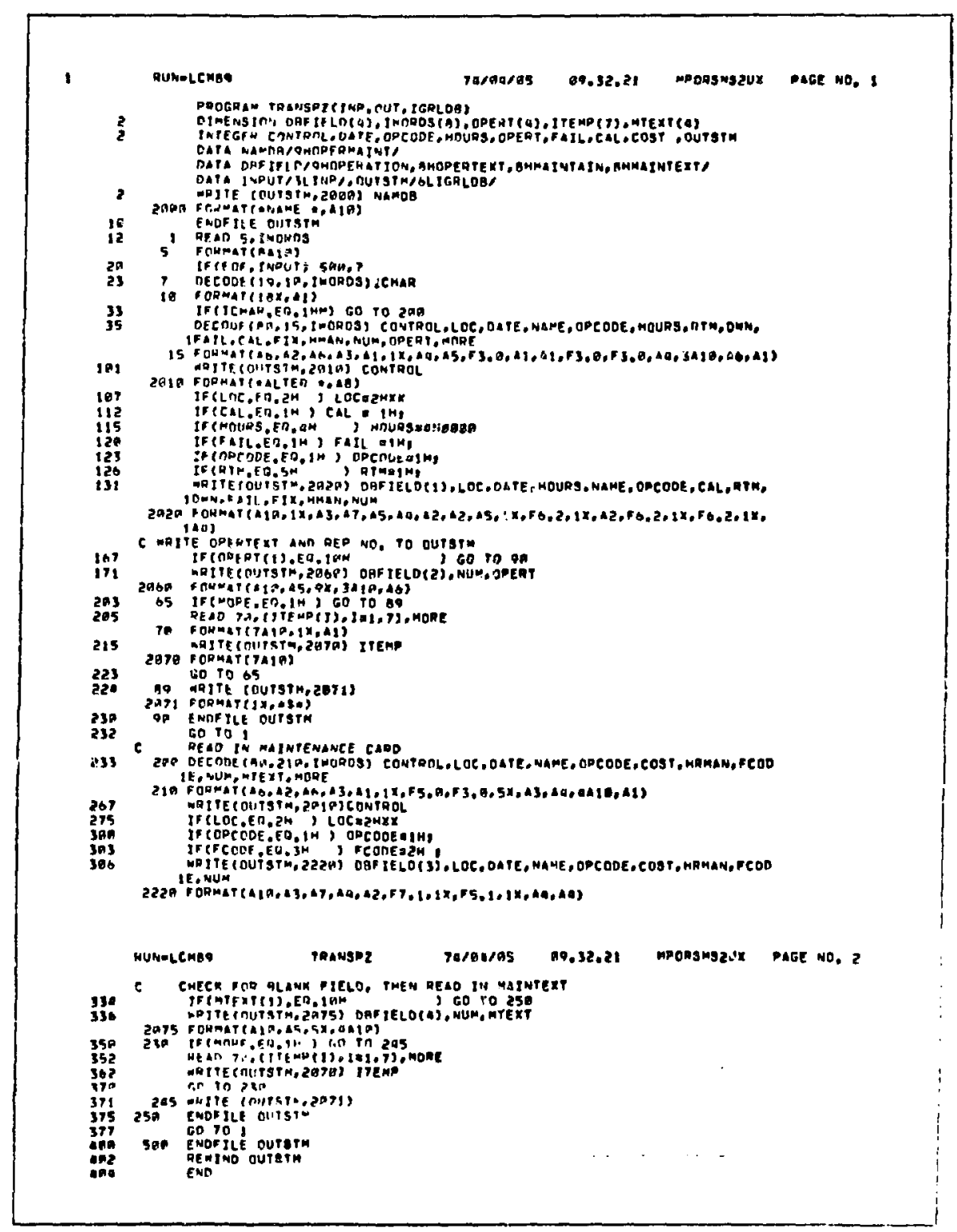

Fig. B.7.a. Operations/Maintenance Data Base. Reads operations and maintenance cards and transposes them into GIRLS style card images. Checks for blank subfields and adds semicolons. Report number is added as first word to text fields, See Figs. 3-4 and Appendix A.2. 


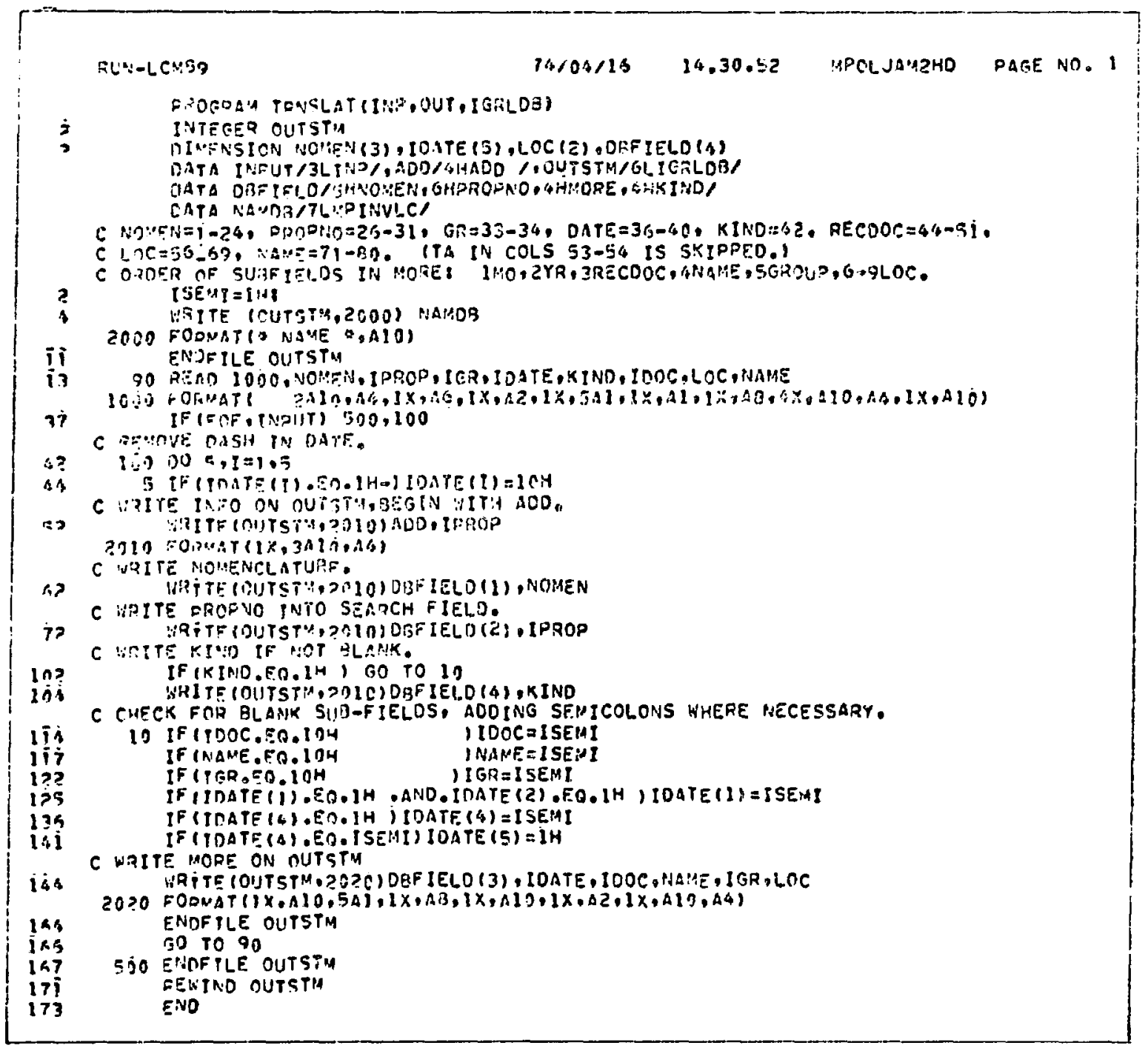

Fig. B.1.o. MP Inventory Data Base. Reads inventory cards and translates then into GIRLS style card images. Date, month, and year are put in to separate subfields after renovirg hyphens. 


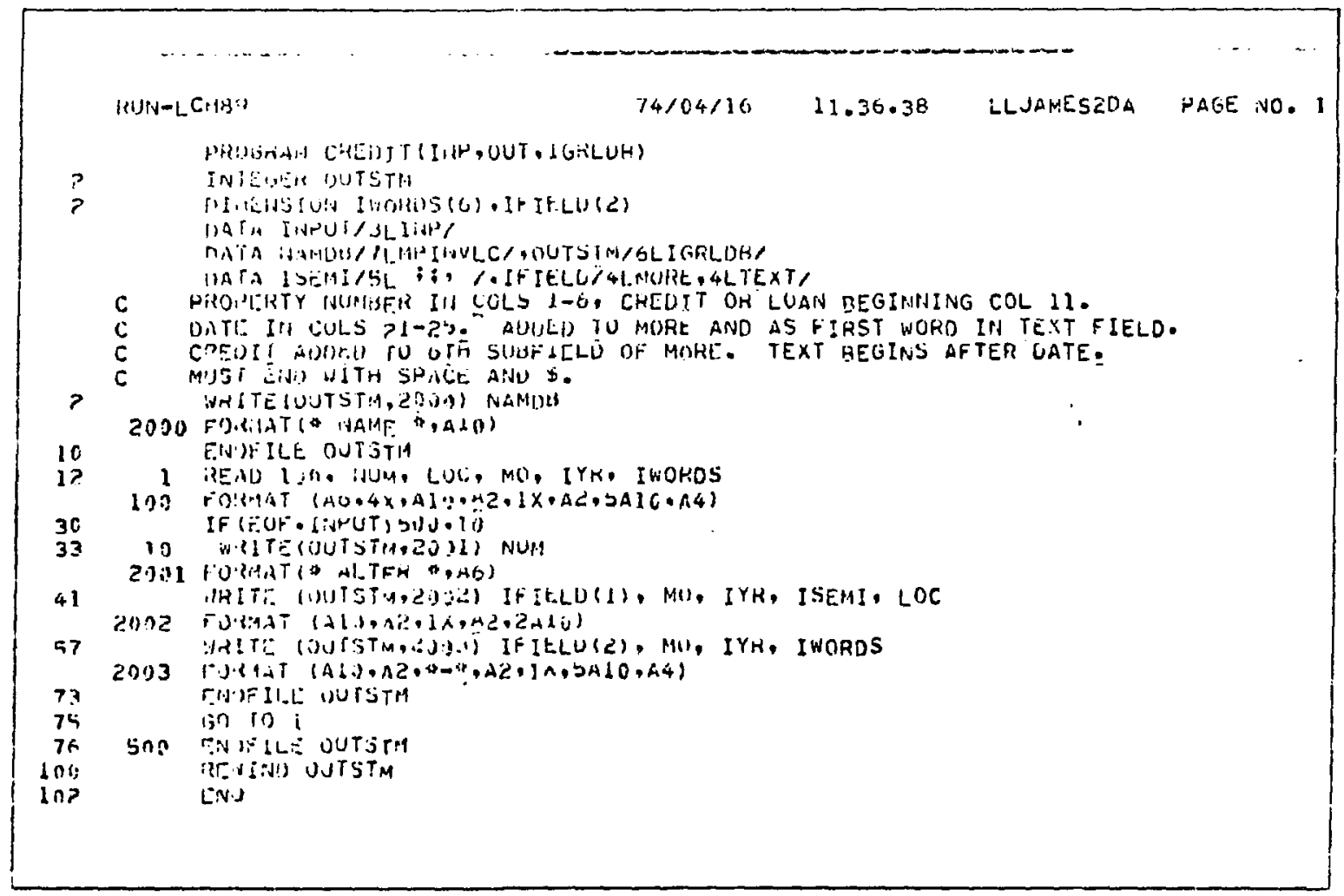

Fig. B.l.C. MP-Inventory Data Base. Simple program to alter items to show removal from MP control when property is sent to salvage, loaned outside the division, or sent out for repair. Text field explains transaction in detail. See Fig. 17 and Appendix A.5. 


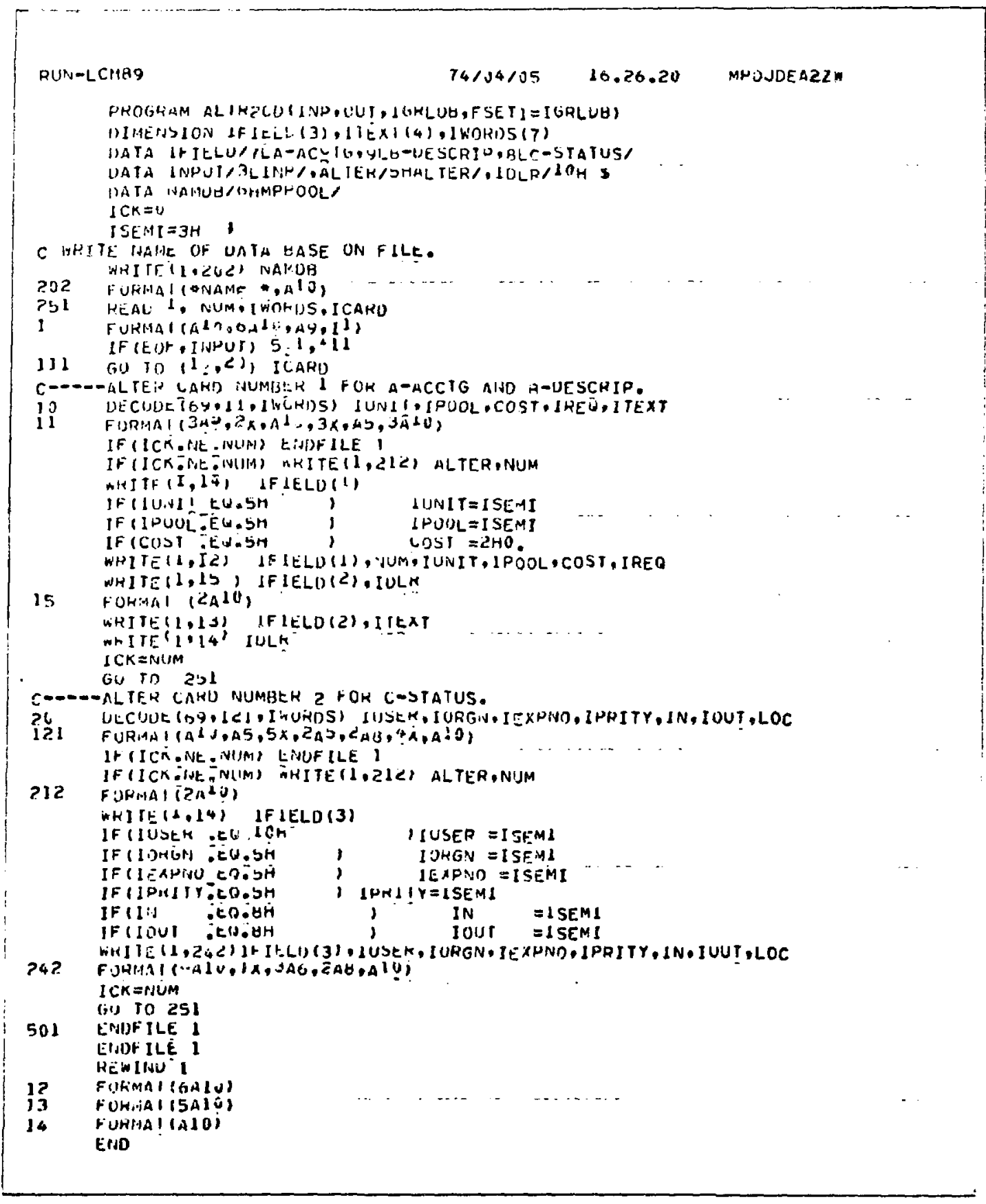

Fig. B.1.d. Experimental Pool Equipment. Reads cards and transposes into GIRLS format to alter items by deleting previous entry and adding updated information. Card form may vary and is signaled by a read-in variable which determines which fields are to be altered. See Figs. 18-19 and Appendix A.6. 
B.2. Post-Processor Programs. Information is retrieved from the GIRLS data-base with commands that are essentially English, for example:

RETRIEVE PRDBLEM ONE

NAME AND GRQUP OR DATE

CRITERIA DNE

NAME EQ JDNES

GRDUP EQ ENGRG

DATE GT 731015

GIRLS provides several subroutines, e.g. NEXTITM, GTFIELD, which allow the user to process the information in a procedural language program, such as FORTRAN, to the desired report format.
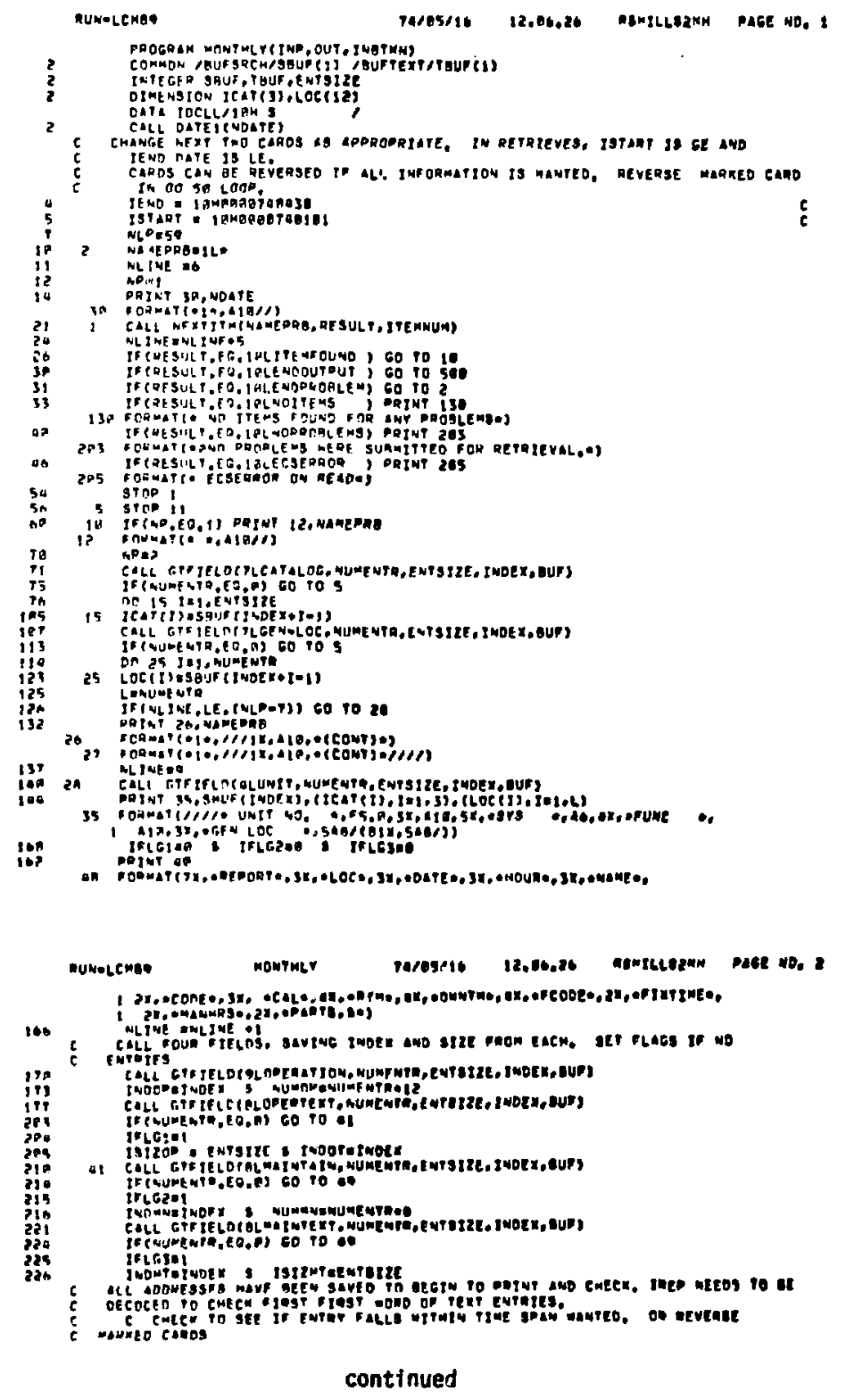

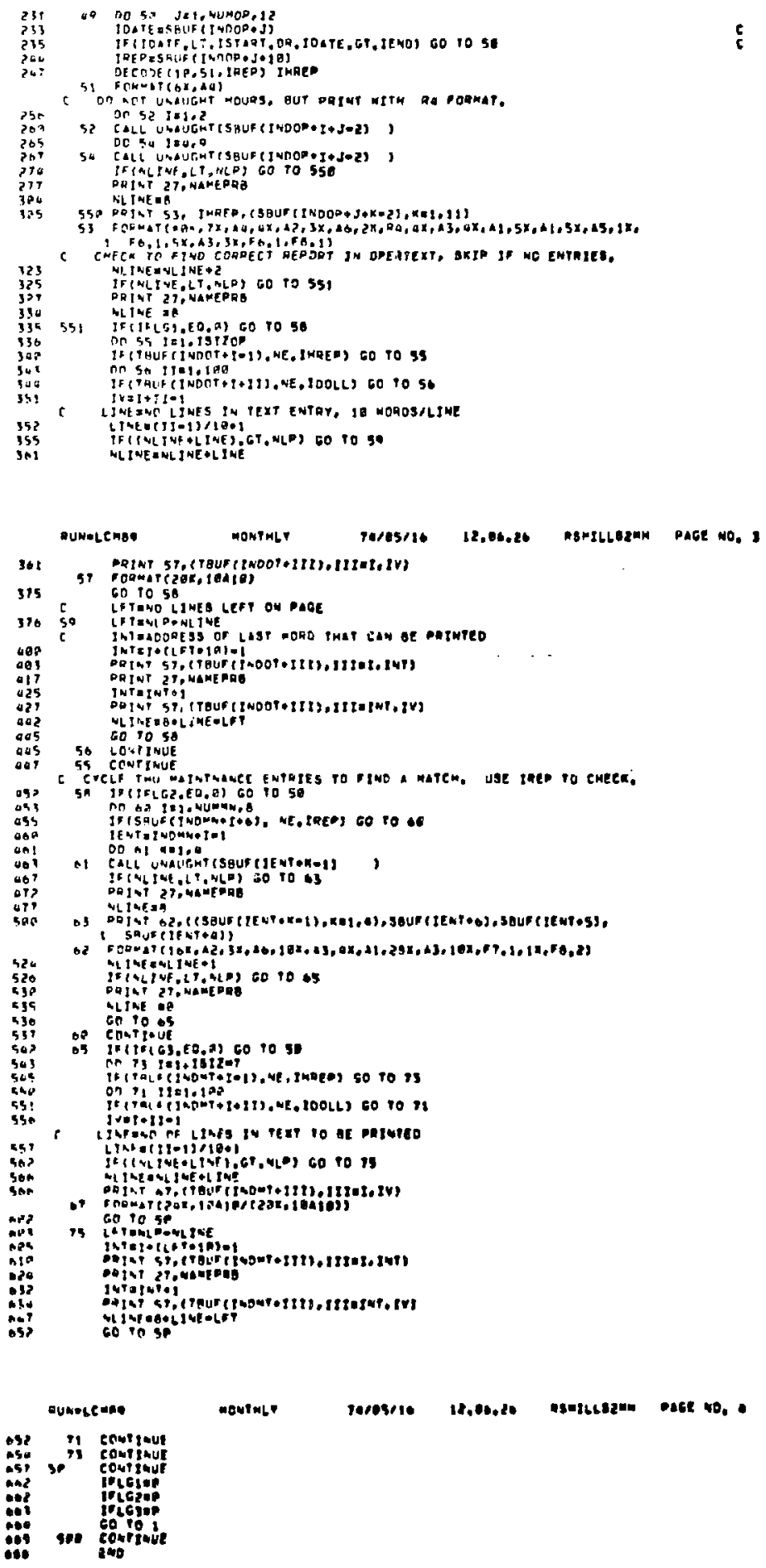

Fig. B.2.a. Operation/Maintenance Data Base. Prints all or selected information for each item retrieved. Mainjenance reports are printed with corresponding operator action. See Fig. 5 and Appendix A. 2 . 


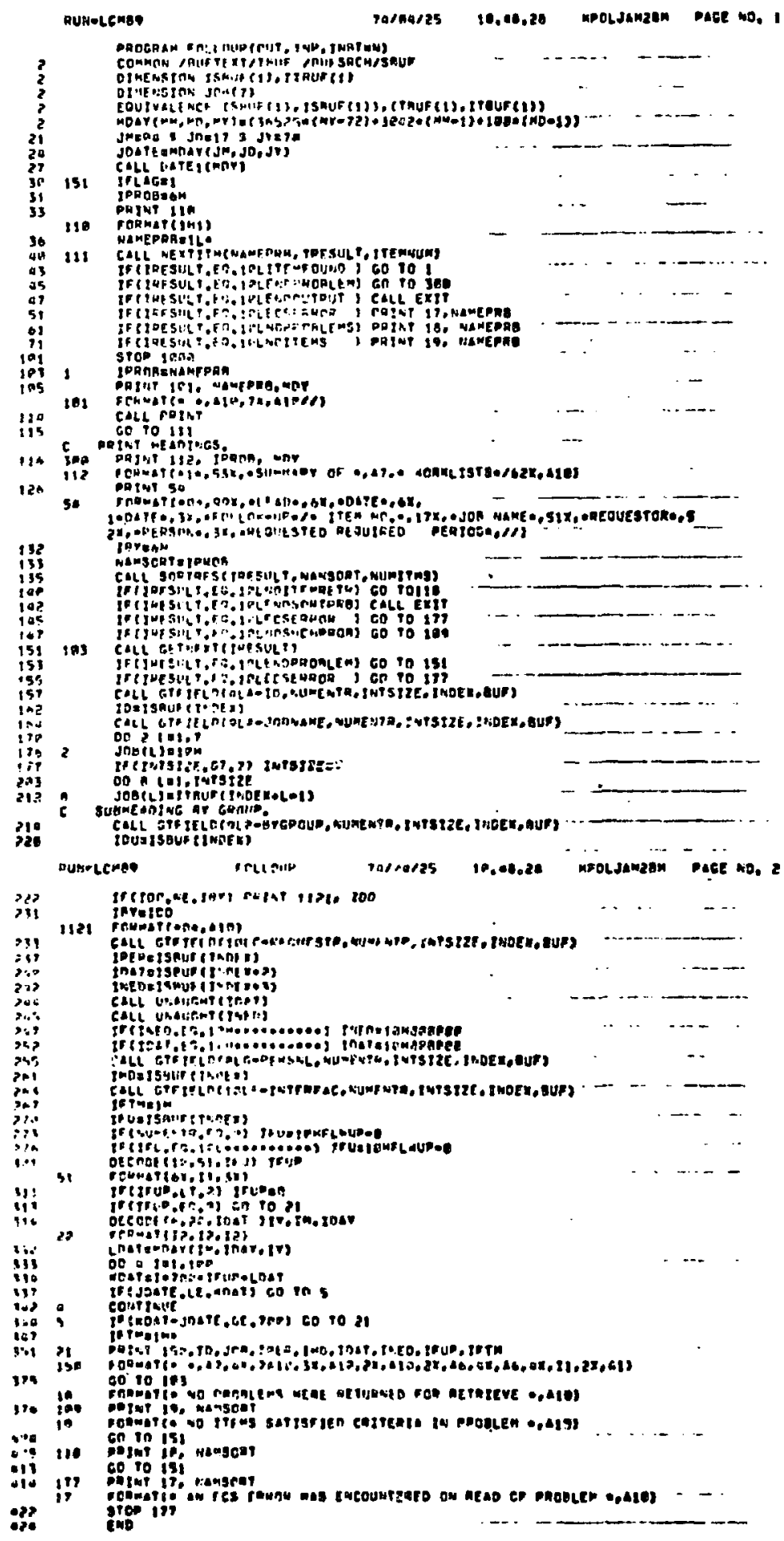

Fig. B.2.b. Work Accounting Followup produces a 1isting, as in Fig. 15, of all jobs for a retrieve with stars after those that are due to be followed up. This is determined by the date requested and the interval specified in that interface. See also Appendix A.4. 


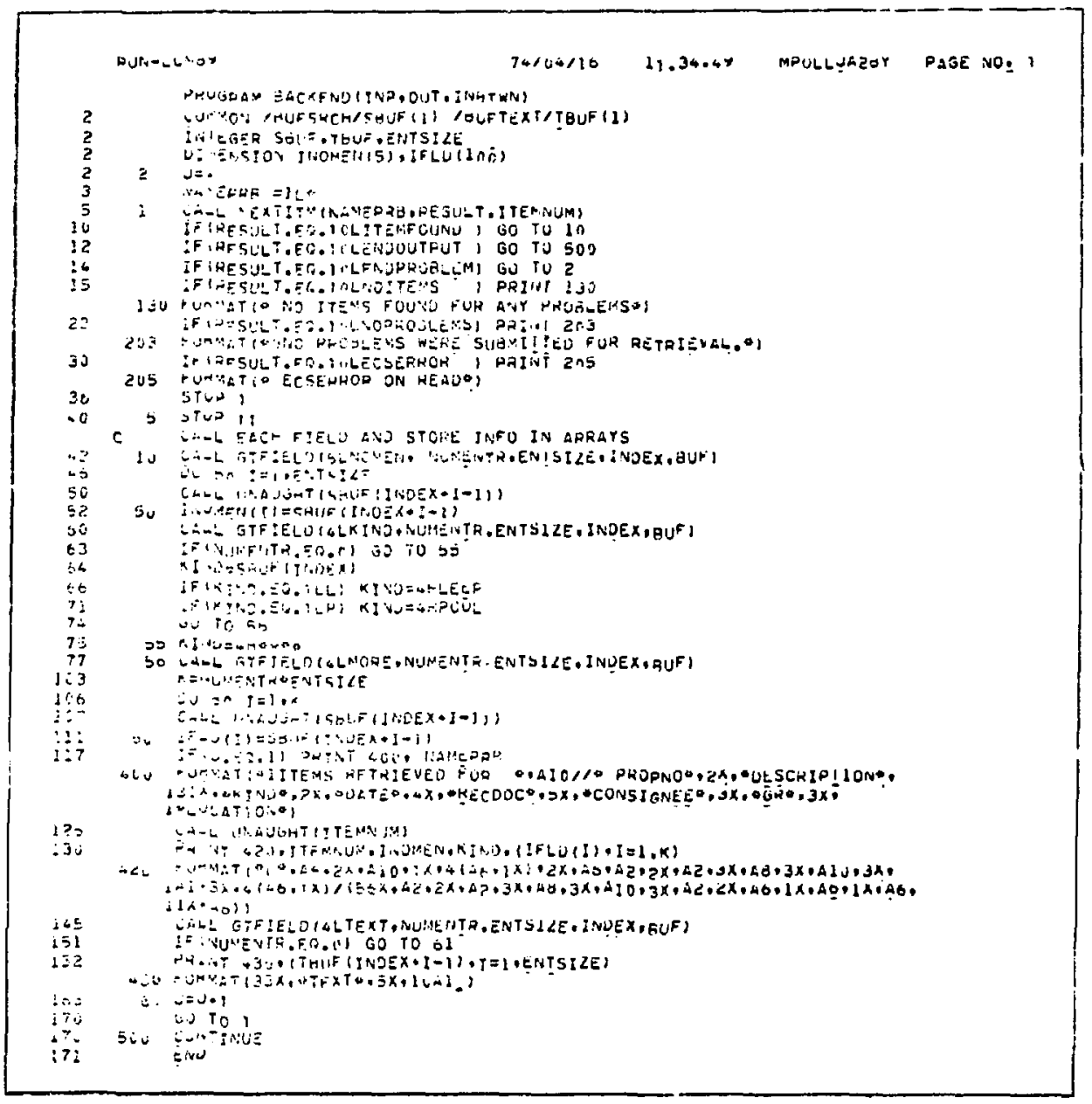

Fig. B.2.c. MP Inventory Data Base. A simple program to print in column form the information stored in the data base. Each search field is called, and infonmation printed after unaughting (removing leading aughts) when appropriate. See Fig. 17. A modification of this program (not included) will print only last entry in an item to show current location and assignee. Also see Appendix A.5. 


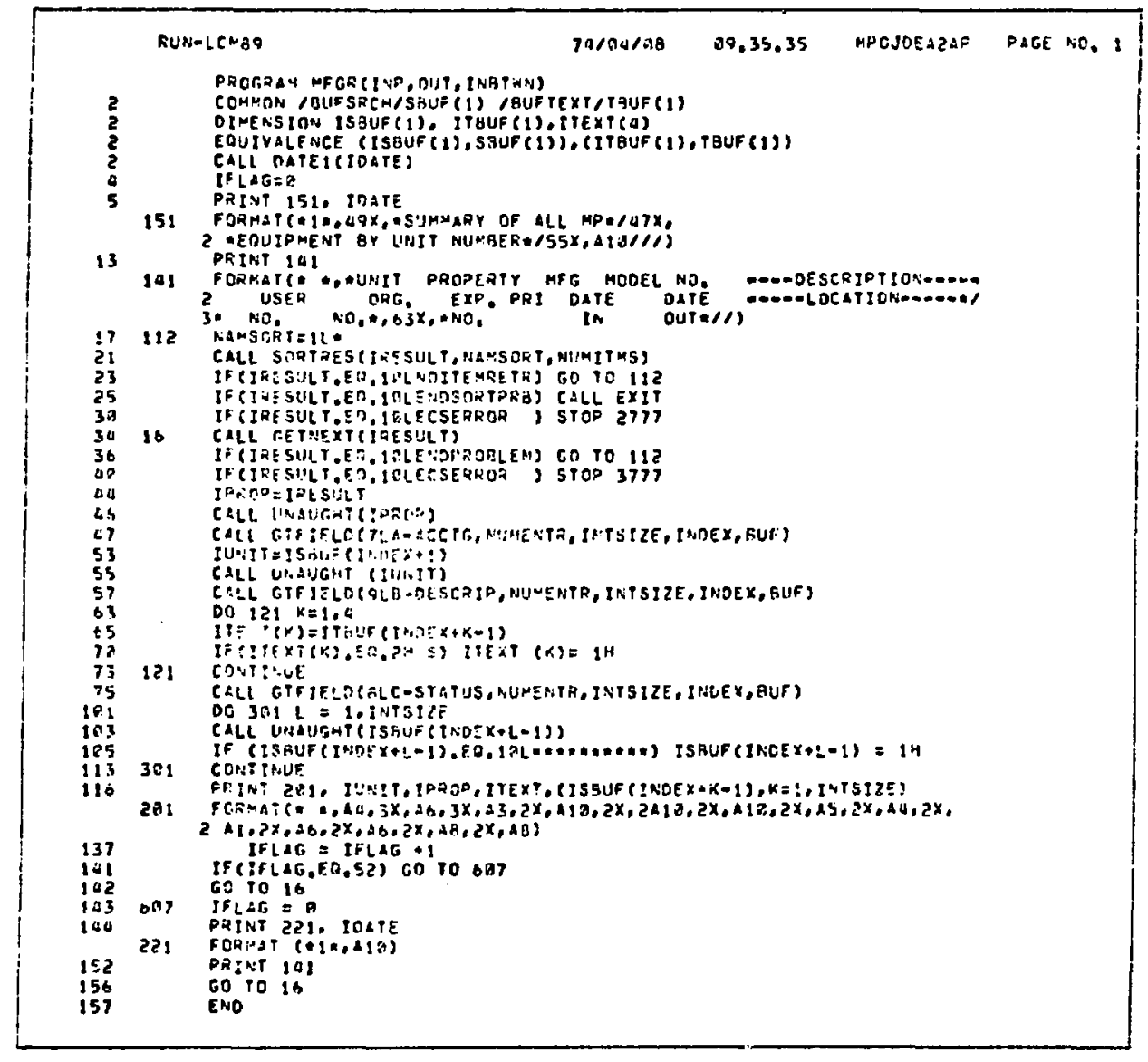

Fig. B.2.d. Experimental Equipment Pool. Prints by unit number all equipment, current location, to whom assigned and for which experiment. See Fig. 18 and Appendix A.6. 
Fig. B.2.e. Experimental Equipment Pool. Program counts total number of each kind of unit, present number in use, and their distribution by experiment number or group. See Fig. 19 and Appendix A.6.

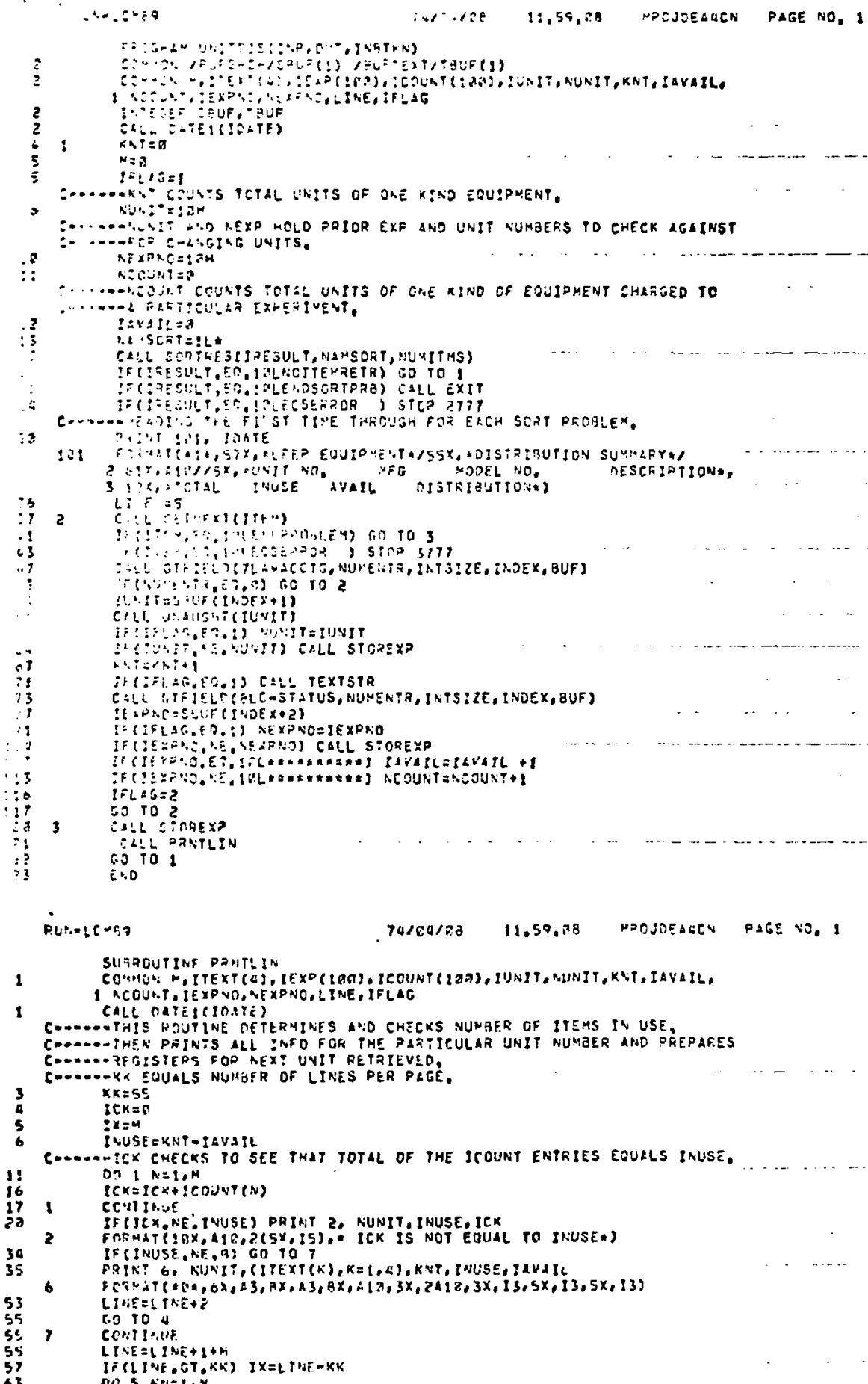

continued 


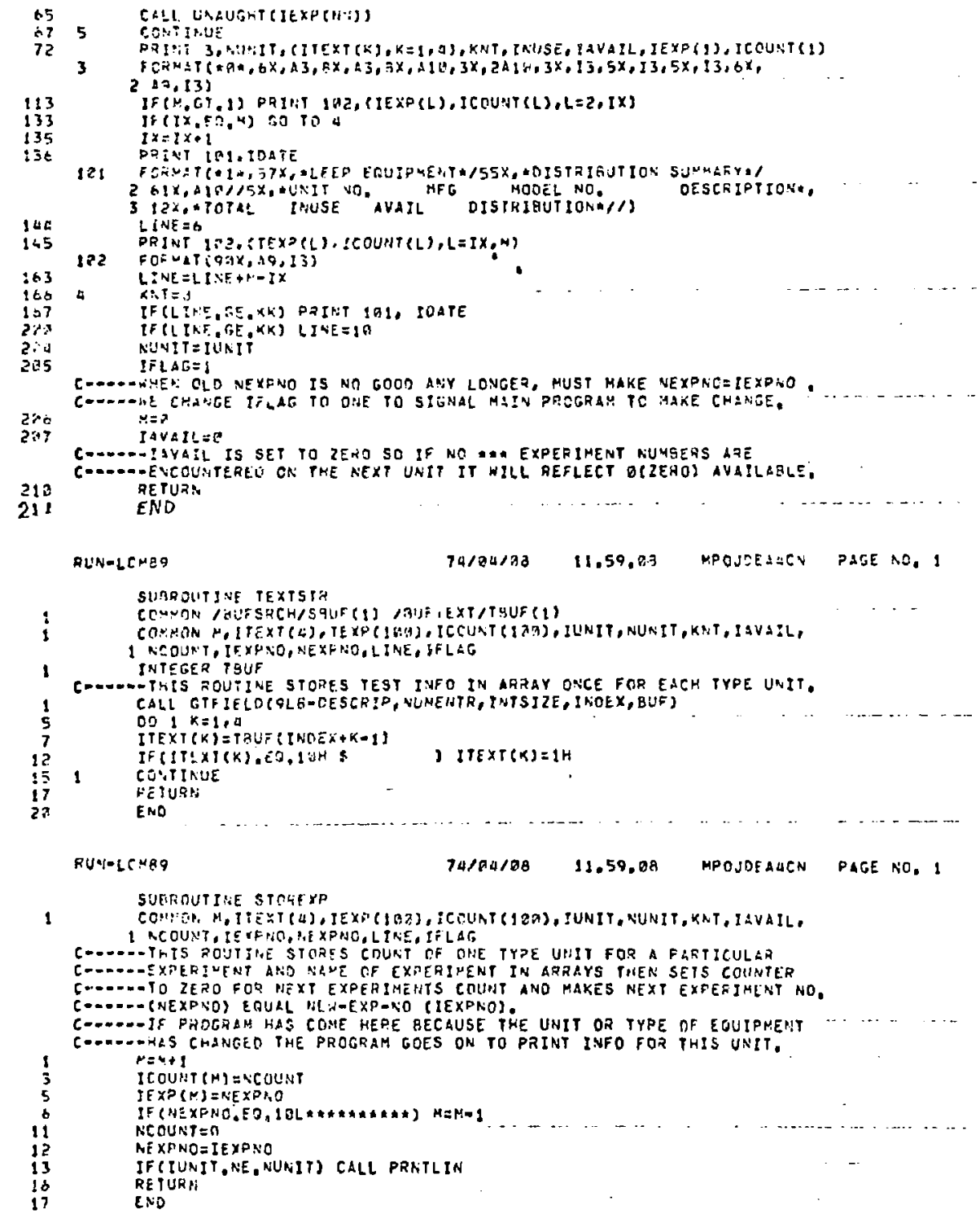


Fig. B.2.f. LAMPF Users Data Base. Depending upon the name of the Retrieval problem, the membership is printed in one of two different formats, with the appropriate heading among the ejght available. See Fig. 20 and Appendix A.7.

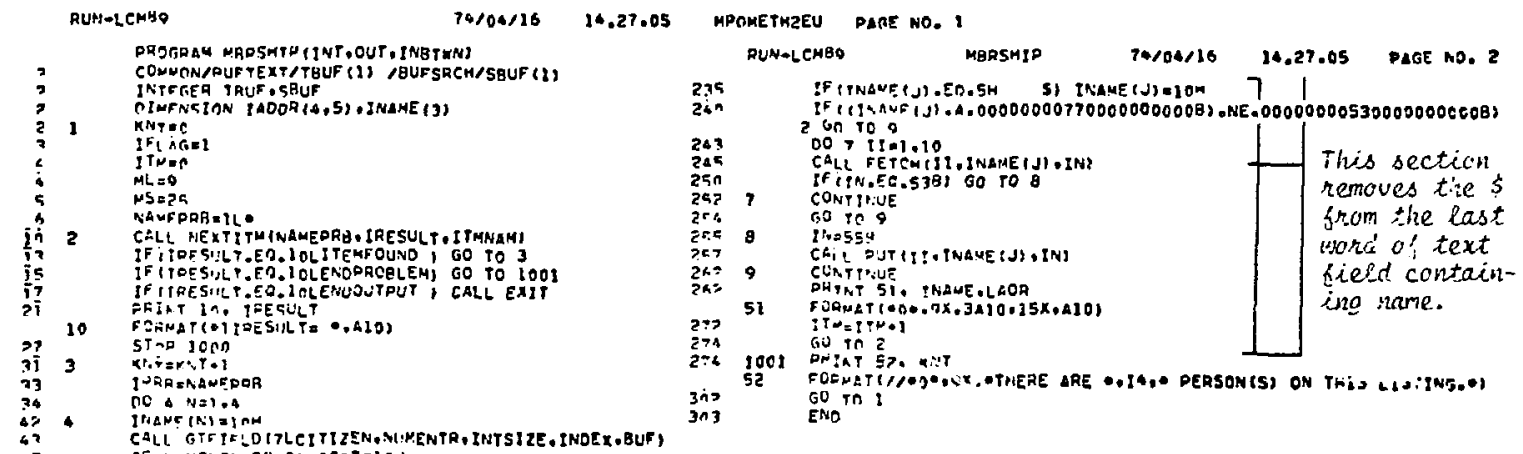

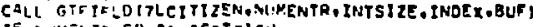

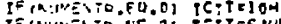

B.jT TETT=SUUF ATNEEX

LIiSI IC!? =13H

25 Fow

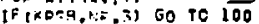

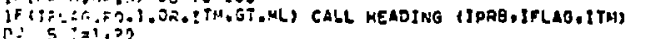

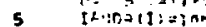

$i_{i=1}^{n=5}$

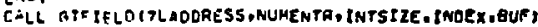

DO II JalijhTslize

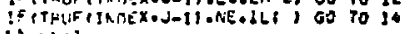

$i x_{1}+\cdots, 1$

ims

14 go in 11

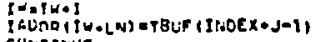

CH:T

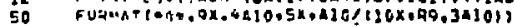

iTh-

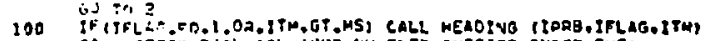

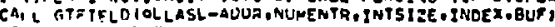

Landeratifitimex)

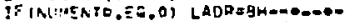

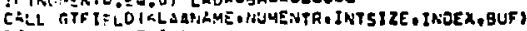

bu 9 Jal. PATSIYS

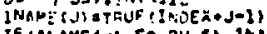

IF IINAME (H,ESTLS SI INAME (J)

RUNOLENGQ 14/04/1h 14.27.05 MPOMETK2EU DACE HO.

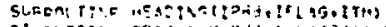

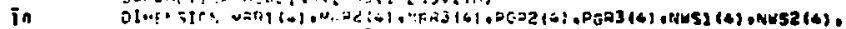

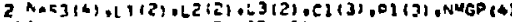

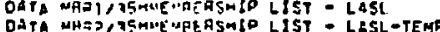

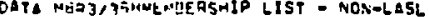

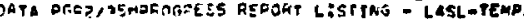

OATA DGJT, TEA

OATA RACI JSW E. SLETTER LISYING - LASL

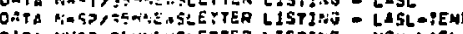

OLTA NLE 3/7SWD:E HSLETTEA LISTING - NONALASL

然

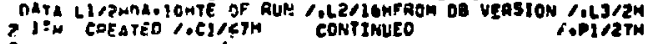

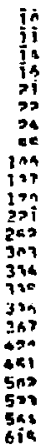

CALL OATE:TIOI

CAi! DATESTINAYE OND,IVI

Ifing

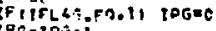

ipú:

Fiflelentofrosi 60 TO 10

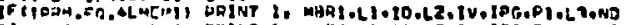

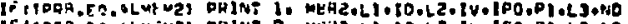

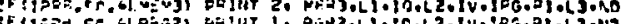

if t1:EH.EC.

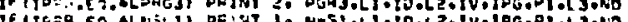

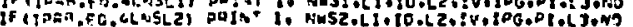

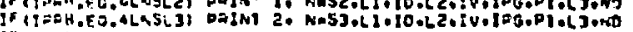

$16,3 r, 5:$

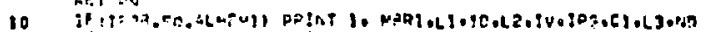

if

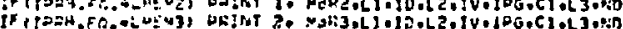

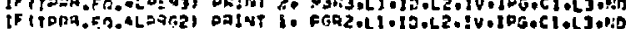

IF

If

if $300+$ in

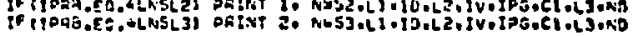

Fonmat for Fig. 20

Alternate Format, not shoin

Headings for page 1 of print

continued

Heading jor ale successive prage headings. 

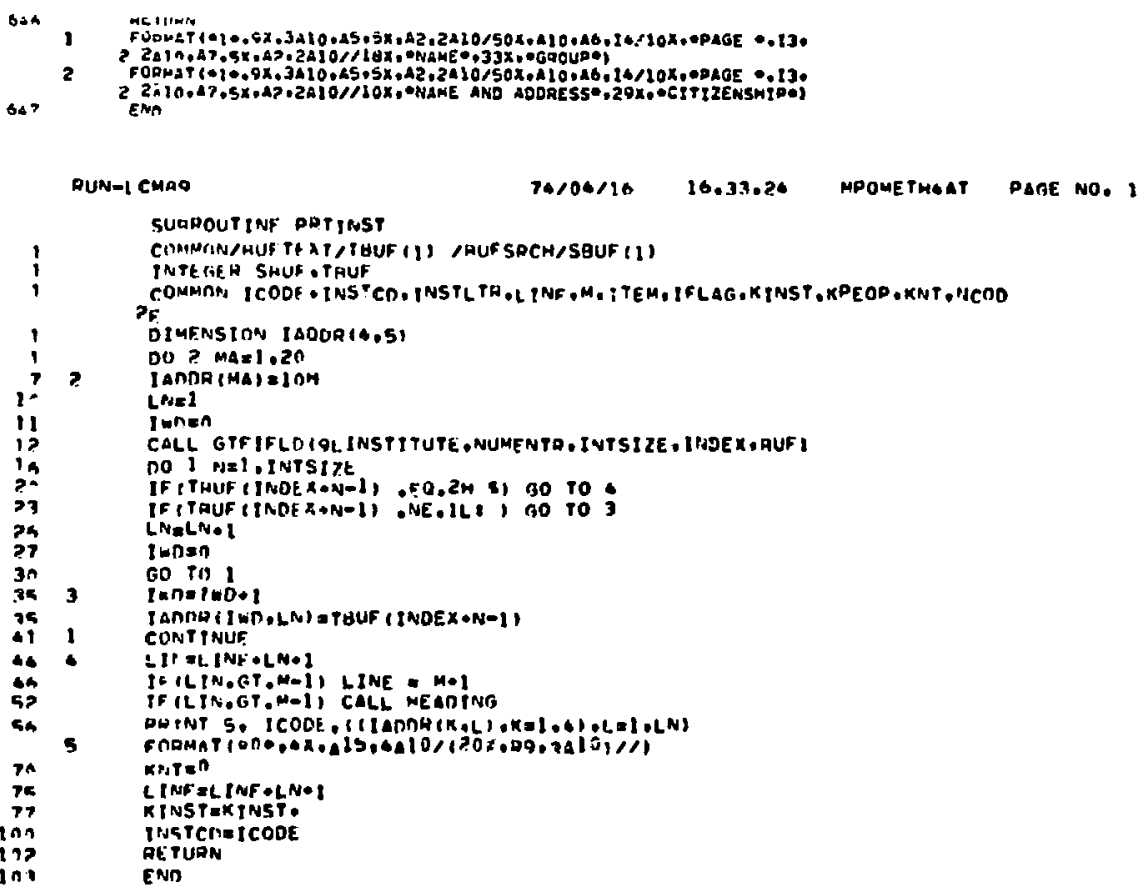


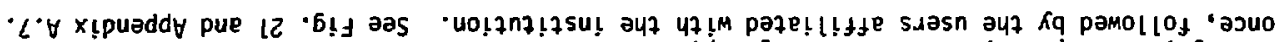

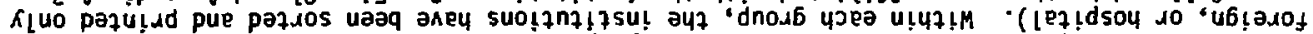
- 


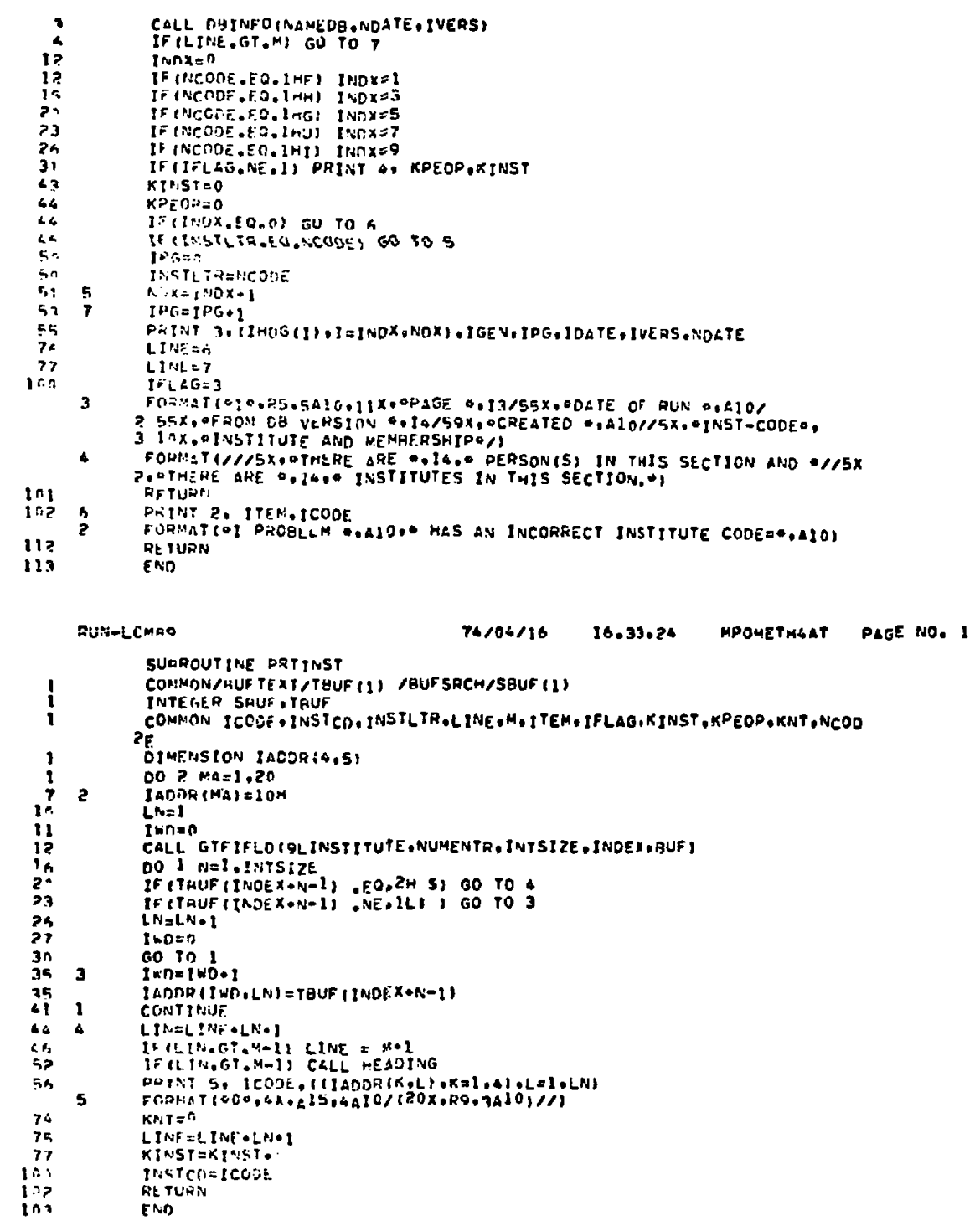


Fig. B.2.h. Experiment Scheduling. Program designed to show the status and spokesmen of all proposals submitted to the Program Advisory Committee for LAYPF. See Fig. 23 and Appendix A.8.

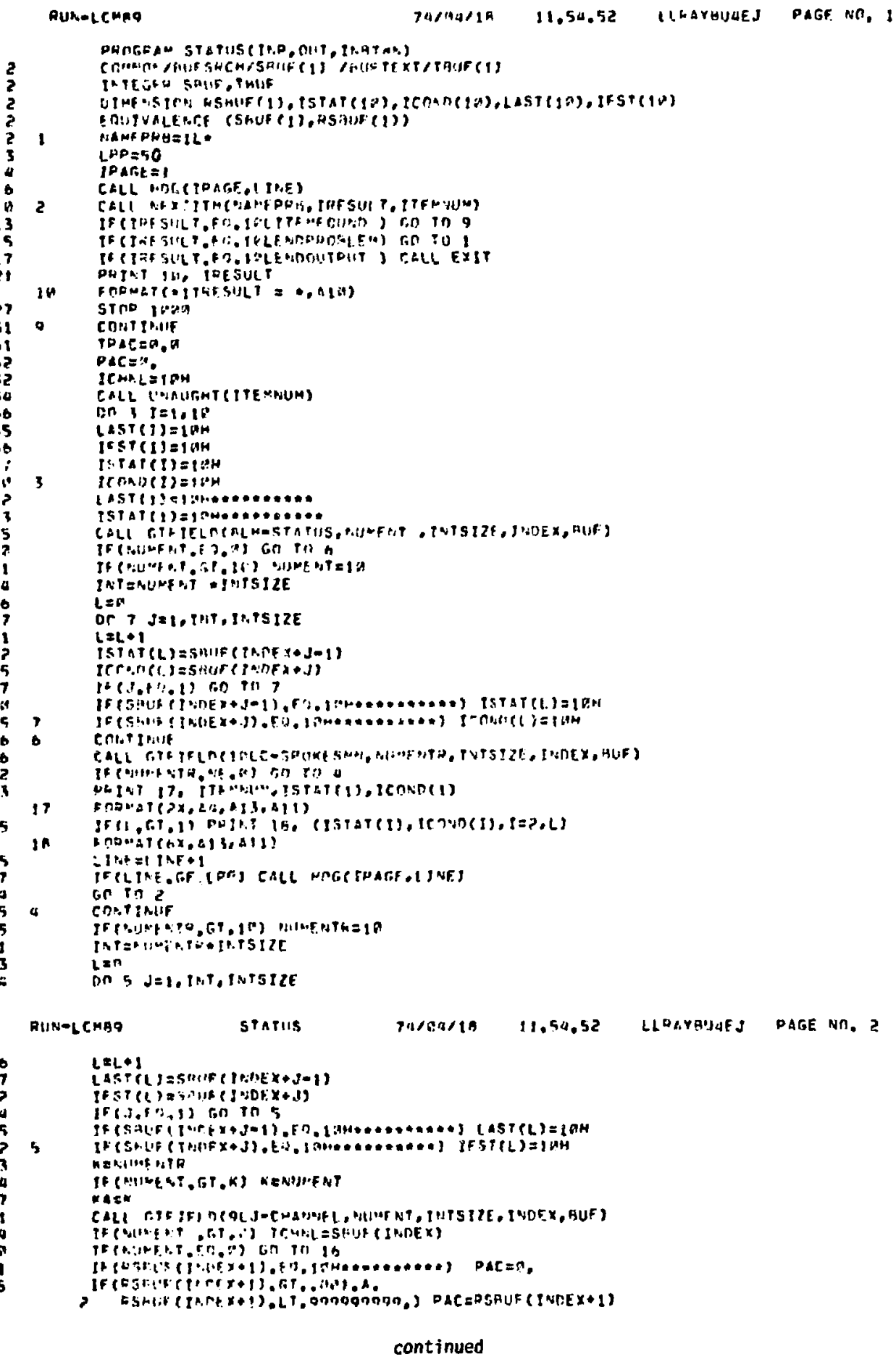

continued 


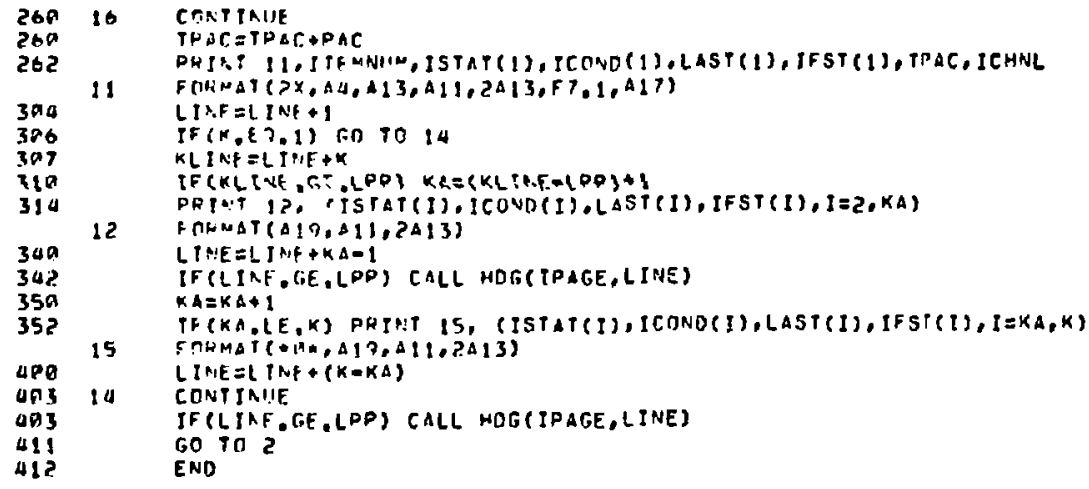

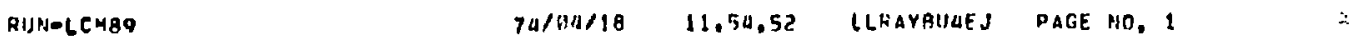

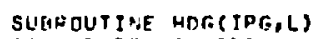

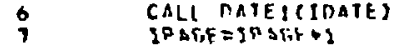

11 PRINT 1, IDATFIOAGF

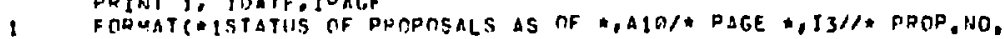

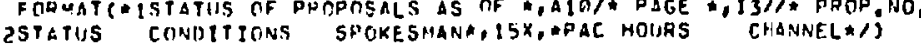

HETURN

ENA 


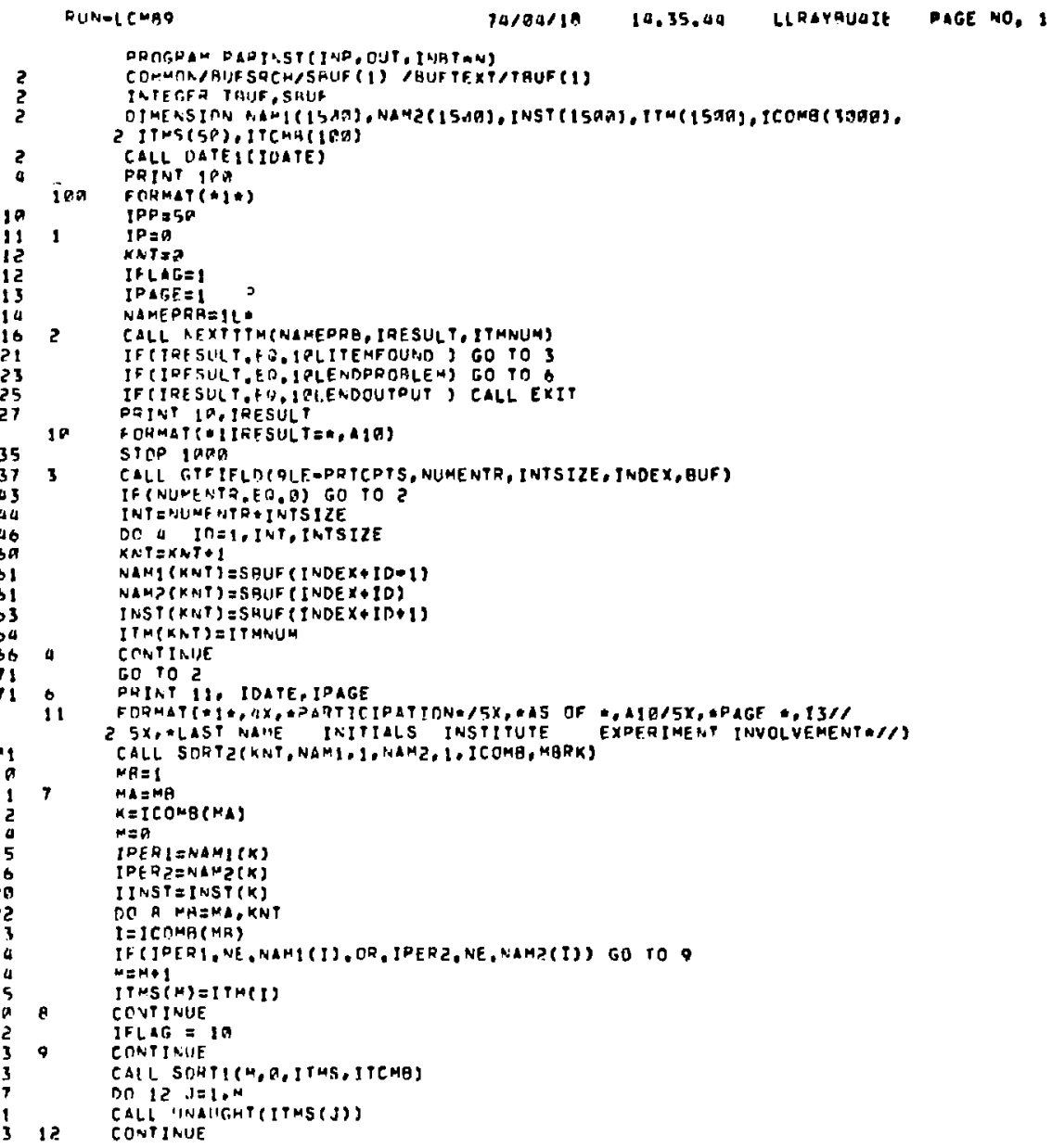

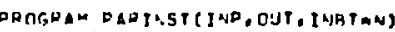

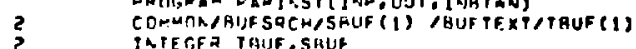

3 TAFGFA TAUF, SHUP

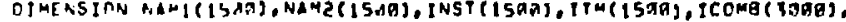

2 ITHS(5P). ITCMA(IBD)

CALL OATEITIUATE)

TOO PRINT IPA

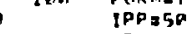

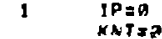

IFLAGE
TAT

IFLAG

IPATE=1

2 CALL AEXTYTM(NIMEPRO, IRESULT, ITMNUNS

IF TIRE 5 LH T. 2 . IPLITEMFOUH, GO 103

IF (IPFSULT,EO.JPLENDPRORLEM) GO TO O

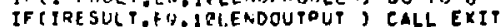

DEINT

19 FOHMAT\{OIIRESULT=MAMIA\}

3 CALL GIFIFLDQQLF-PRTCPTS, NUMENTR, INTSIZE, INDEX, BUF)

If (NUMENTZ,EO,B) GO TO 2

INTENUMF NTR I INTSIZE

DC \& In=1,INT,IMTSIZE

XNT $T=X M T+1$

NAMI (KNT) $=S$ BUF $(I N D E X+1 D-1)$

NAMP $(K N T)=$ SBUF $($ INDEX+1D)

INST (KNT) $=$ SAUF IINDEX $+I D+1)$

ITM(KRT) $=$ IT MNUM

4 CONTINI)

- paint id, idote,jpage

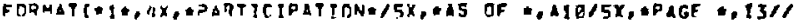

$25 x_{2}$.LAST NA" INE INITIALS INSTIPUTE EXPERIMEN" INVOLVEMENT "/

CALL SORTZ(KNT, NAMI, 1, HAMZ, L, ICOMB, MBRK)

$M A=1$

, MRE $\quad M A=M B$

$K=I C O N B(M A)$

$M=B$

TPER! $=$ NAMI $(K)$

IPEREZNANZ(K)

IINST $=$ INST (K)

DC $A$ MHEKA,KNT

$i=I$ COMBC(MR)

IF(JPERI.NE, NAHI(I), OR, IPERZ,NE, NAM? (I)) GO TO

$m=m+1$

TTMS $(H)=1 T M(T)$

a covisnue

IFLAG $=10$

CONTINUE

CALL SOHTICH, C, ITHS, ITCMOS

DO $15, J=1, \mathrm{M}$

cali inalightstims (3))

1? CONTINUE

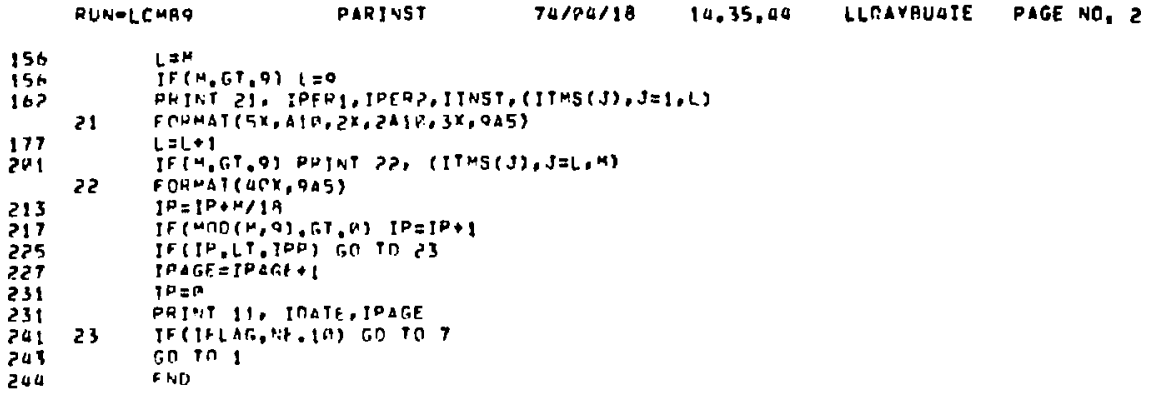

Fig. B.2.i. Experiment Scheduling Data Base. Program which sorts participants, prints their names, institutions, and all proposals with which they are involved. See Fig. 24 and Appendix A.8. 
B.3. Pre-Processor Programs that have been produced from Post-Processor Programs.

Fig. B.3.a. Summary of Operating History. This post-processor is run once a month to summarize, graph, and store the total activity for the month. The information is taken from active items in OPERMAINT. Bargraphs are printed and the data is stored on tempurary tape in GIRLS ALTER format. At this point, OPERMAINT is released and another data base, GRAPHDATA, is secured. The ALTER tape is read then as input to GRAPHDATA where the statistics are stored permanently to be retrieved later for film graphs. See Figs. 9-11 and Appendixes A.2 anc A.3.

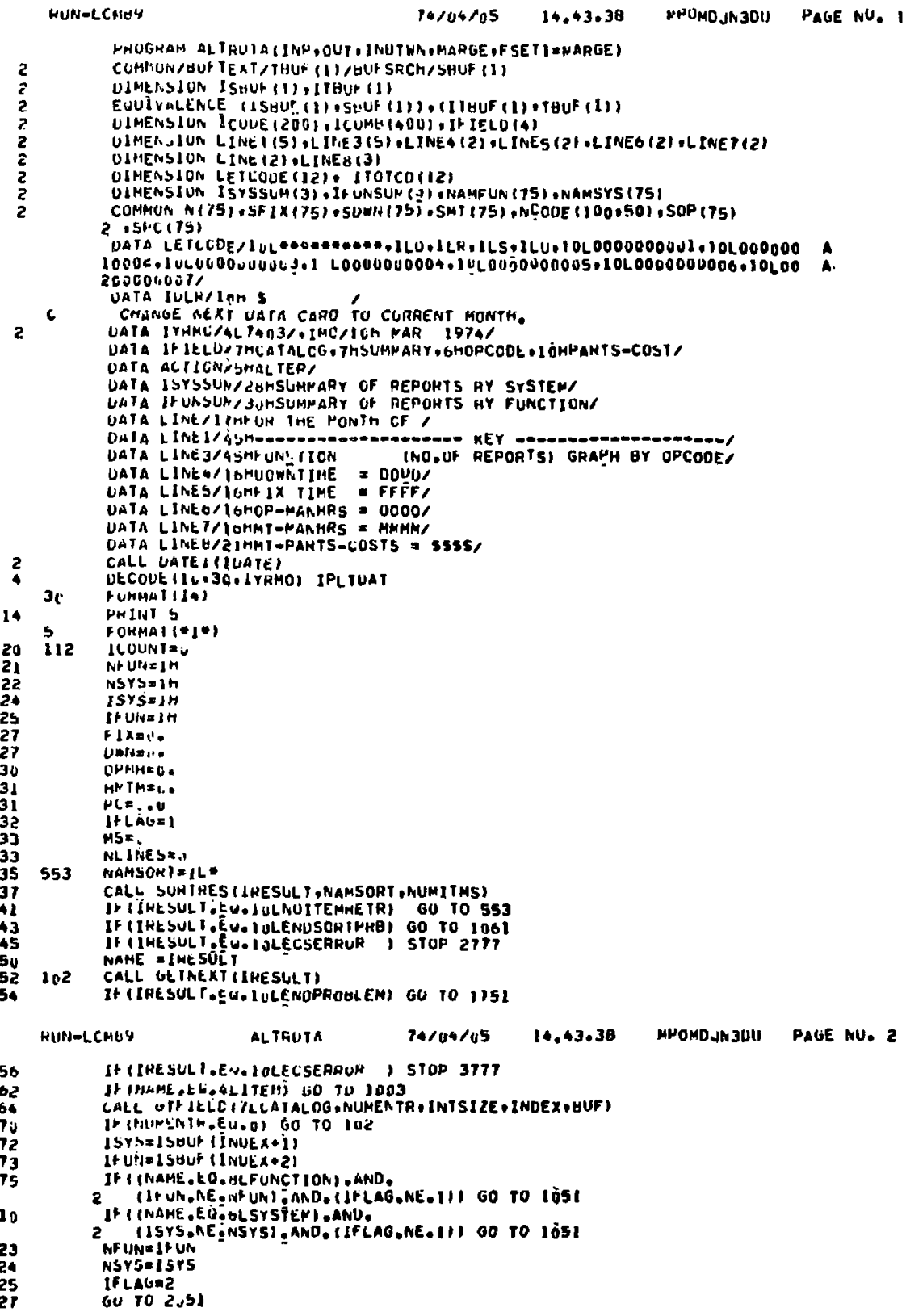




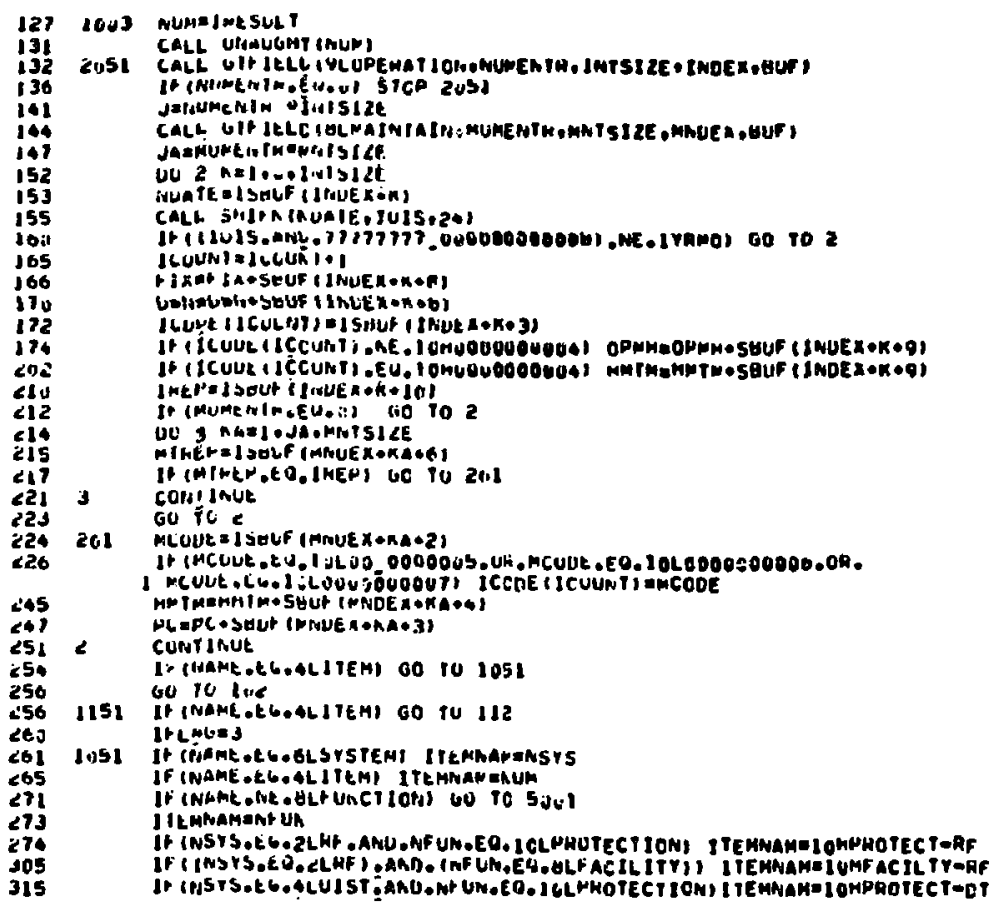

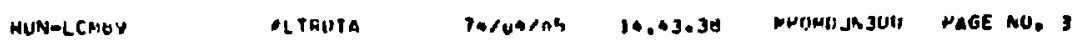

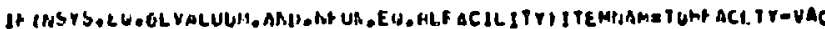

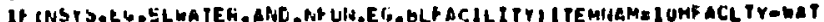

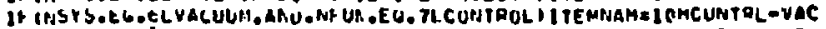

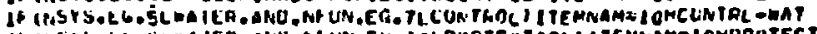

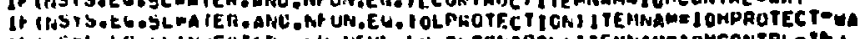

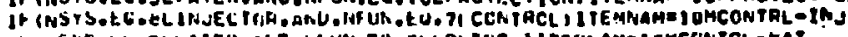

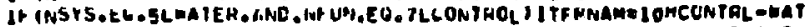

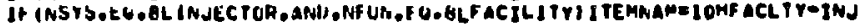

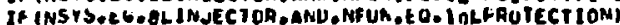

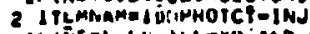

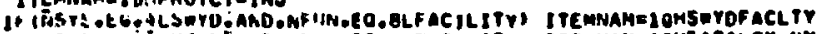

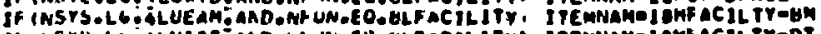

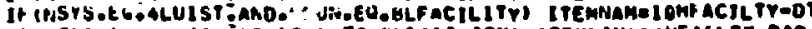

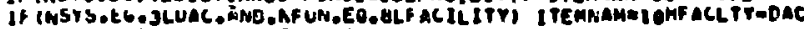

SuOL WhItE II OLUIII ACTIOA I TEMNA

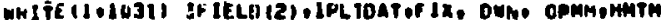

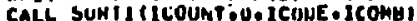

ve io ITLIBId

I TOTCUITG).

10 Cutitinue

LECoI

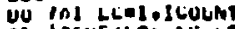

If ICQUE ILCI NE.LETCOOE (LEC) GO TO 700

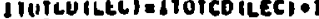

60 To 701

700 LECELECH!

IH ILELGUT-121 00 in 750

fol complnut

WHITE II +769) IF IELO(3) INLTDAT I ITUTCO

ToO PUMAAT (ABAIS.1214)

25 WHITE (1 OCSS If IELYSOI ITHAO.PC

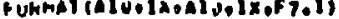

enve ILt 1

Vu $>1$ lasel: Jum

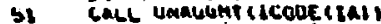

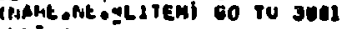

JCUUnt=i

F $1 \times \mathrm{A}_{1}$.

untiar.

oumerio

Mist Man.1.

PCe 1.04

It InahtiotGoaldTERI 00 To 102

NSYS: $15 Y S$

Nib uite If urt 
panu+7uos

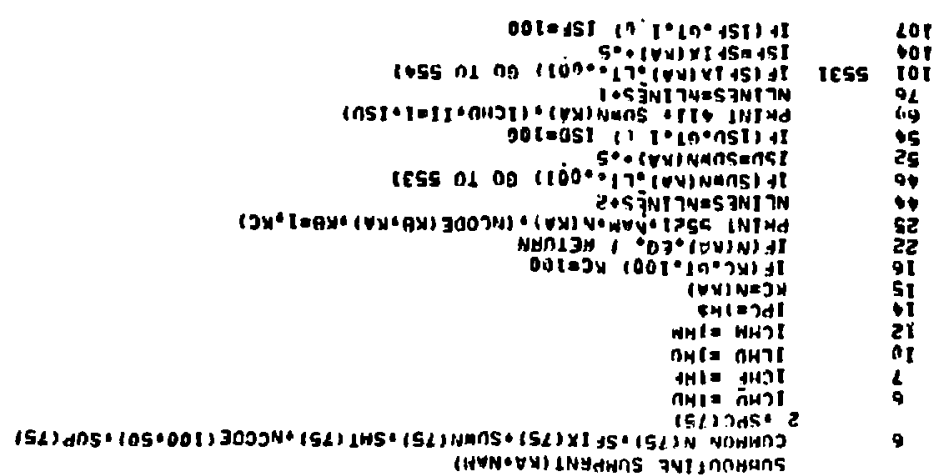

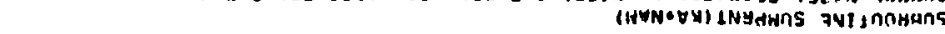

GAH T-NกM

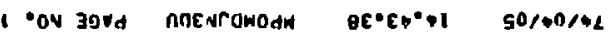

กบ7

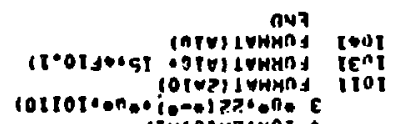

tent

9501

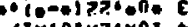
iviotivexnit

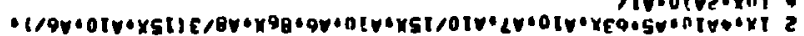

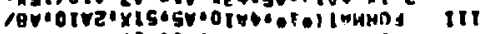

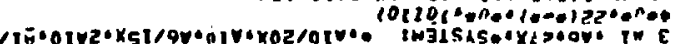

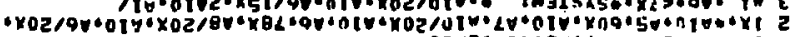

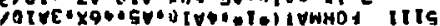

11ชว 7าพ

TกTMYM

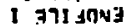

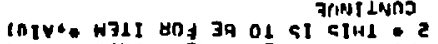

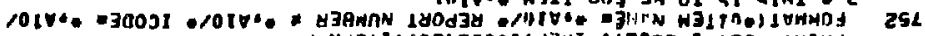

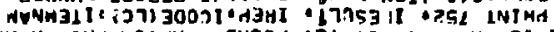

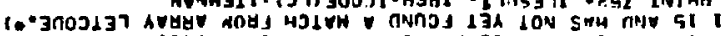

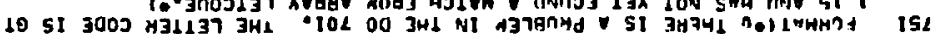
tot

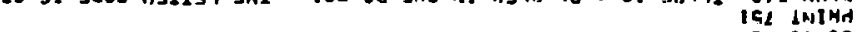

$>$ II กI ถ⿻ हองทา1

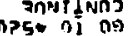

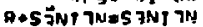

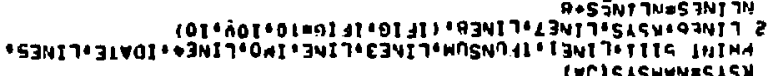
tent ant ivC)SAchunaslen Cos 7PII TN ones of no

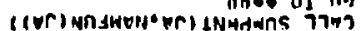
(10 T)

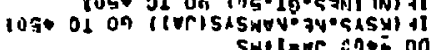

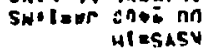
Dogs 3NI Th $>1101$ a9

ระกบามป anNT IN⿴囗十

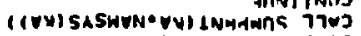
SW I I

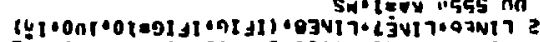

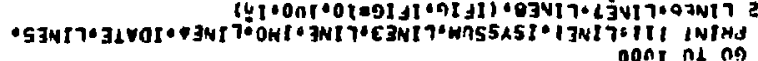
(y) $30021=$ I vnogt ot tove itaisisas

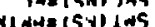
hithe (sw) Ans

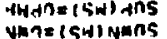
XI $1=1541 \times T$ is

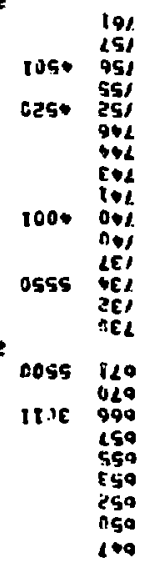

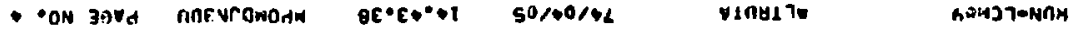

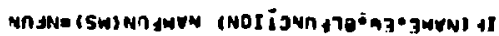

SASVE CGAISLSWWA

dunnst $=15$ in

21101 on

$\cos 20$

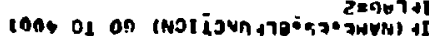

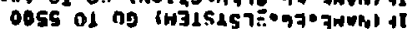

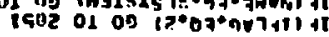




\begin{tabular}{|c|c|c|}
\hline $\begin{array}{l}112 \\
110 \\
133 \\
136 \\
141 \\
144 \\
162 \\
165 \\
170 \\
173 \\
176 \\
114 \\
217 \\
265 \\
224 \\
232\end{array}$ & $\begin{array}{r}511 \\
+11 \\
5521\end{array}$ & 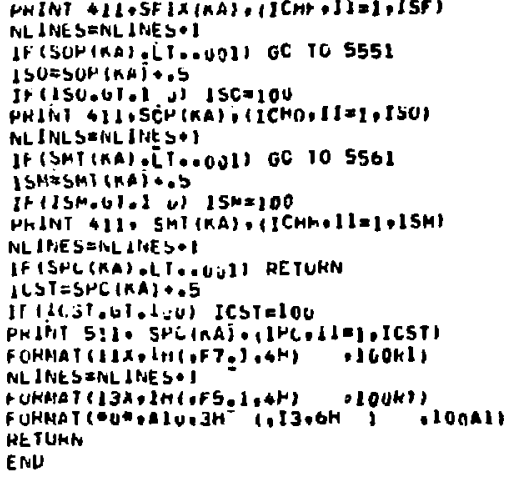 \\
\hline
\end{tabular}


Fig. B.3.b. Pre-Processors can write the retrieval program from data which i's used also in the PostProcessor. This program is one of several designed to analyze failure reports for LAMPF. Reports can be made for specific locations and/or time periods. Data cards are read by the Pre-processor which then writes the retrieval program onto card images in GIRLS format. The same information is written on a temporary file and used by the Post-Processor to select only those entries which satisfy the criteria desired. (A data management system which returned only these entries which satisfy retrieval criteria would eliminate many of the complex Post-Processcirs now needed. Such systems are becoming available on today's market.)

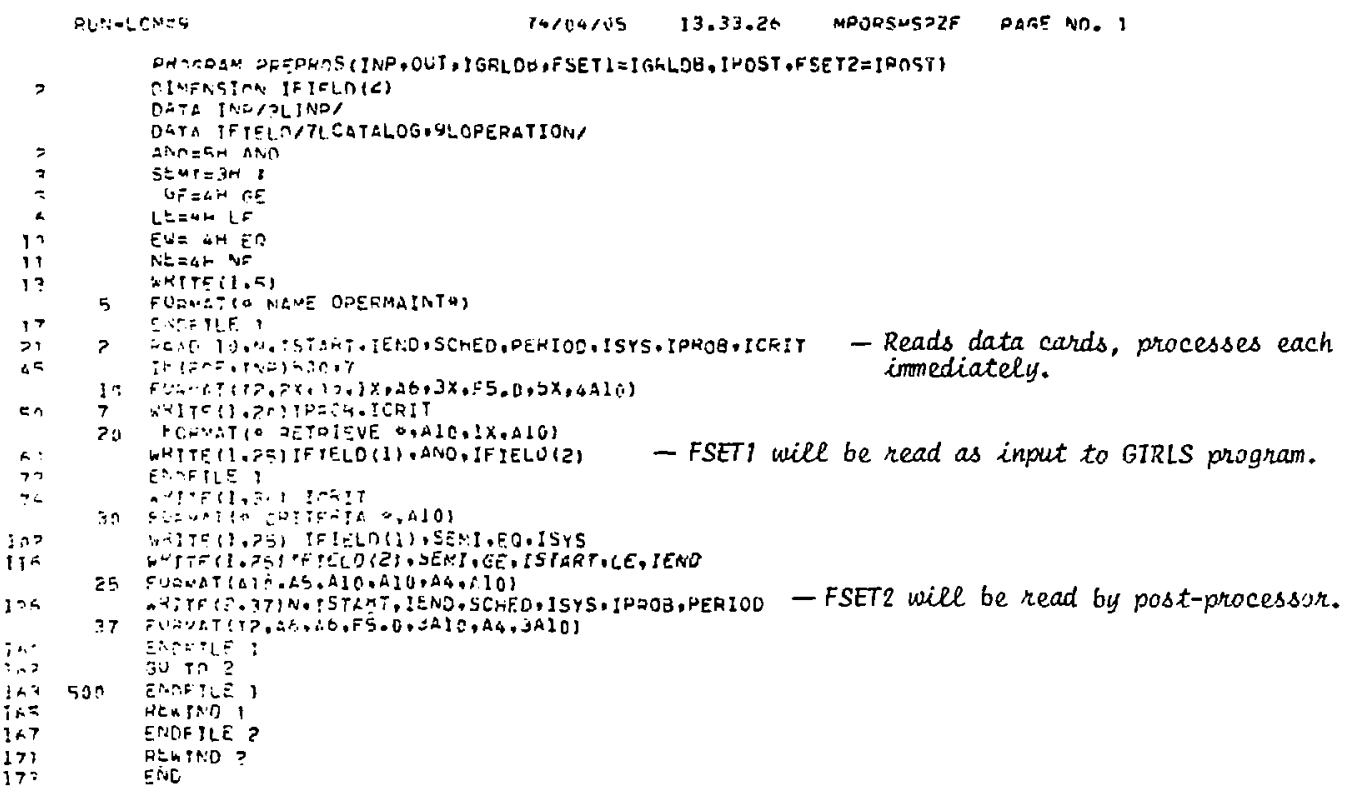

$$
\text { RUP-LCMES 14/C4/US } 13.33 .25 \text { MPOOSMSZZF PAGE NO. } 1
$$

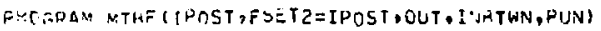

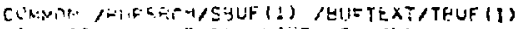

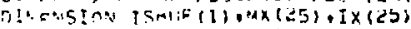

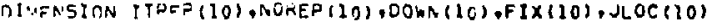

E(UTVALFNCF IT SHUF (1) OSRUF(1))

INTEREF ENTSITE

MACK $=333333333333333333330$

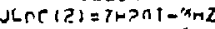

Ji.CC $(7)=$ GM SNJPCE

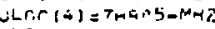

$\therefore L C C(5)=2 M A L L$

C $M=D U A N T J M E \rightarrow M=F I X T I M E-2$.

IHQNT $=1$ IR

$N=0$
$M=A$

1 FFAM(D,5)]FIAG ISTART, IEND.SCHED, LSYSTEM, IPHOD, IPERIOO

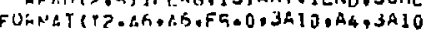

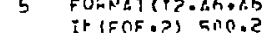

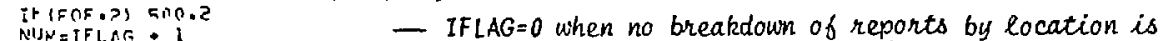

LGC $(1)=7$ RUNCNER wanted. IF $L A G=4$ when wanted and MUM becomes 5 which

DI $3 I=1$, BuM

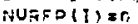

DURN(1) $=0$.

indili=r.

3 ITFO(I)=n

ERPORE $(19.4$.NATART)MASK, ISTART Adds leading aughts to correspond to dates as stored

ENCORE $(10.4$-NFFU $M$ MASKIEND $]$ by GIRLS.

4 FUARAT(BS+AB)

NAMESRB $=0$

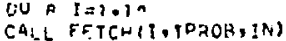

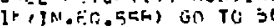

CQLL PIJTII. NAMEPRH, INI

a Cunthue

9 CAHI NEXTITM (NAMEPRZ, RESULT, ITEMNUM)

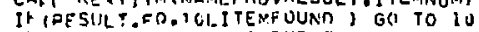

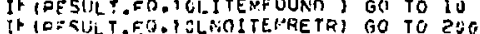

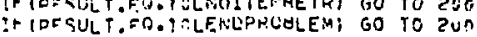

continued 


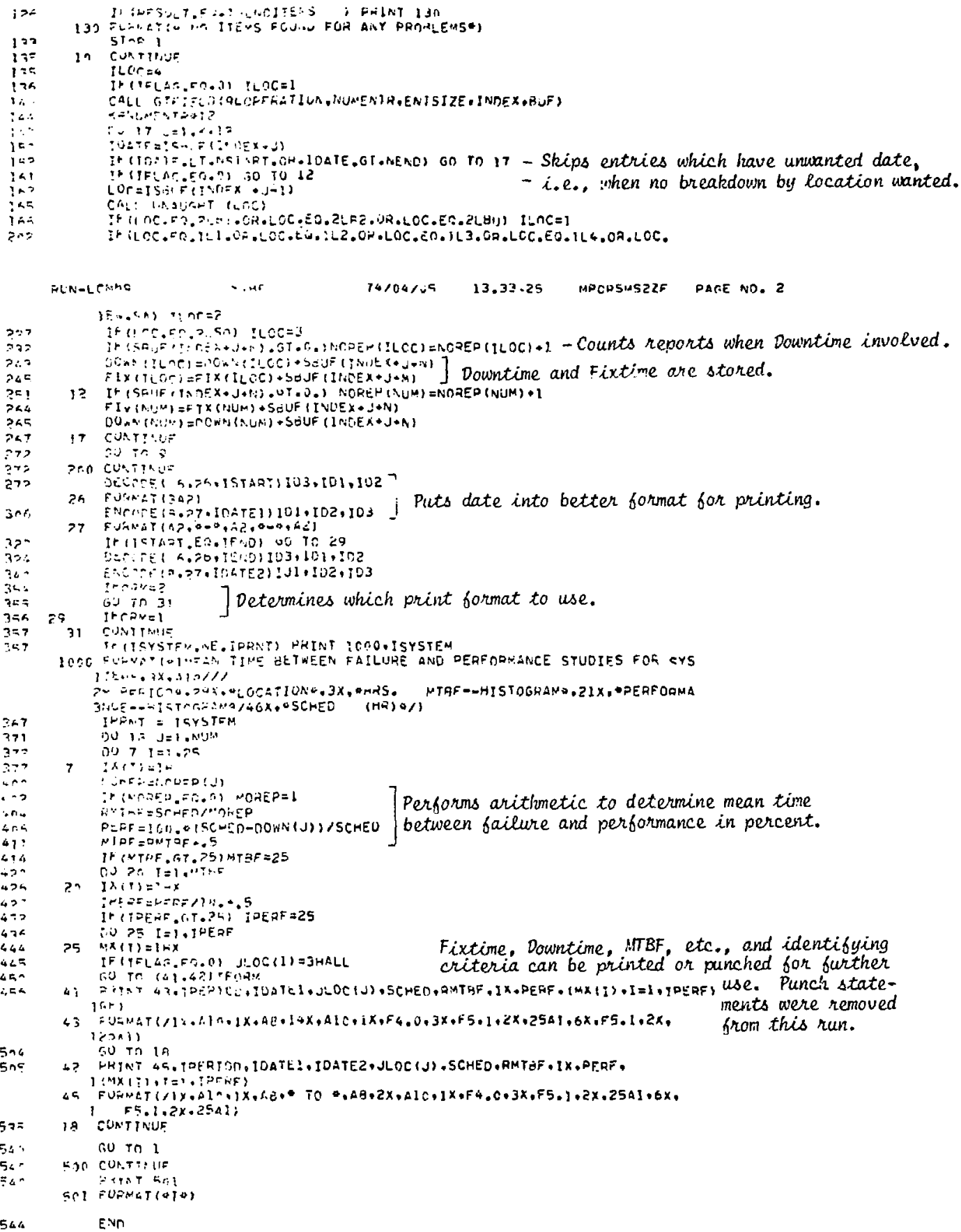




\section{B.4. iliscellaneous.}

Fig. B.4.a. Office and Lab Space Data Base. Retrieved items have been sorted by a GIRLS subroutine and are printed with this program. Variable LINE is used to store information until all fields have been called and is used as a "print positioner." See Appendix A.9.

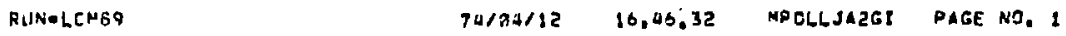
stored until end of entry (\$) is found. 
Fig. B.4.b. Daily Operations/Maintenance Report. Program for PDP-11 to read maintenance cards, search data files, and print information from stored catalog and from the card. See Fig. \& for output.

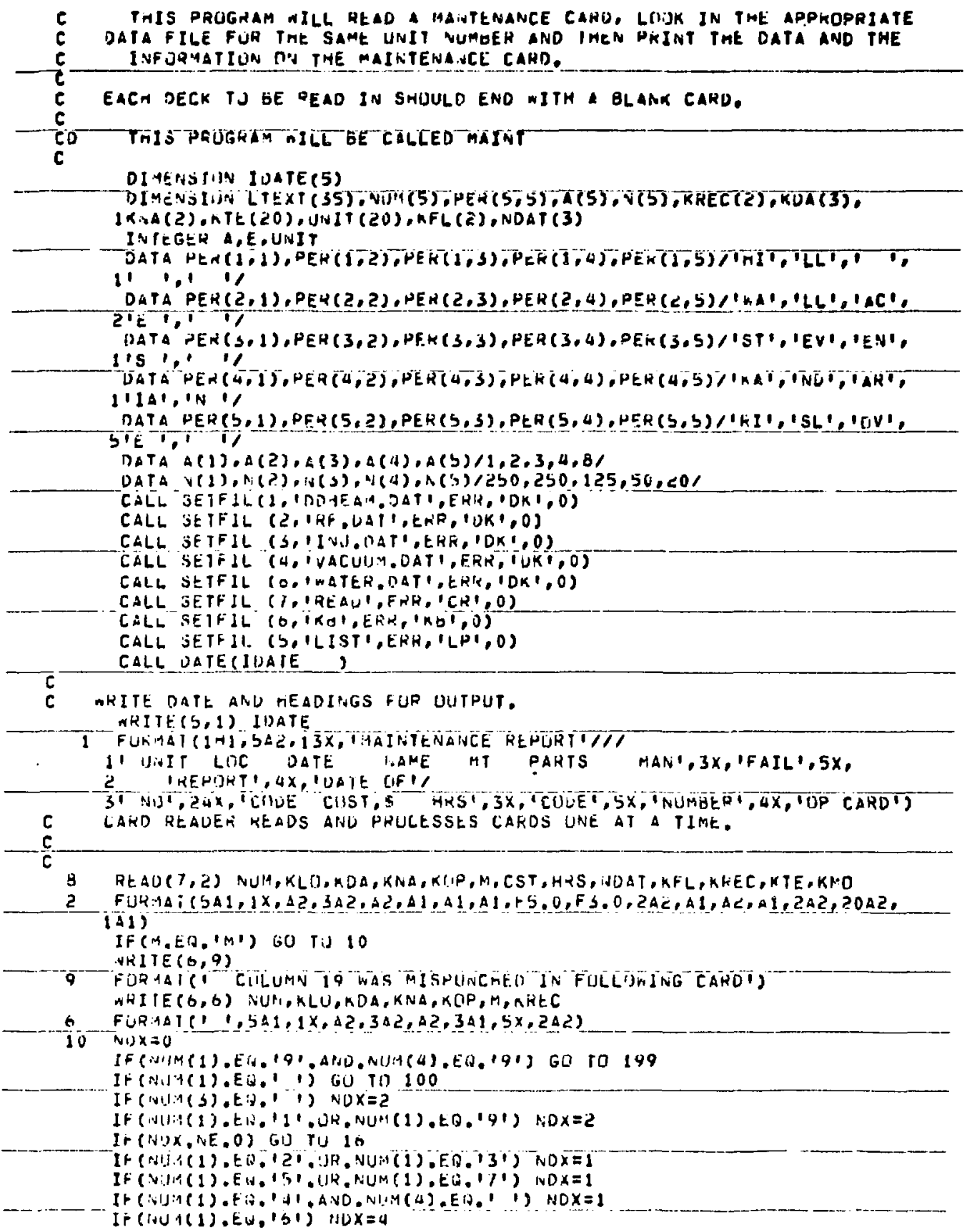




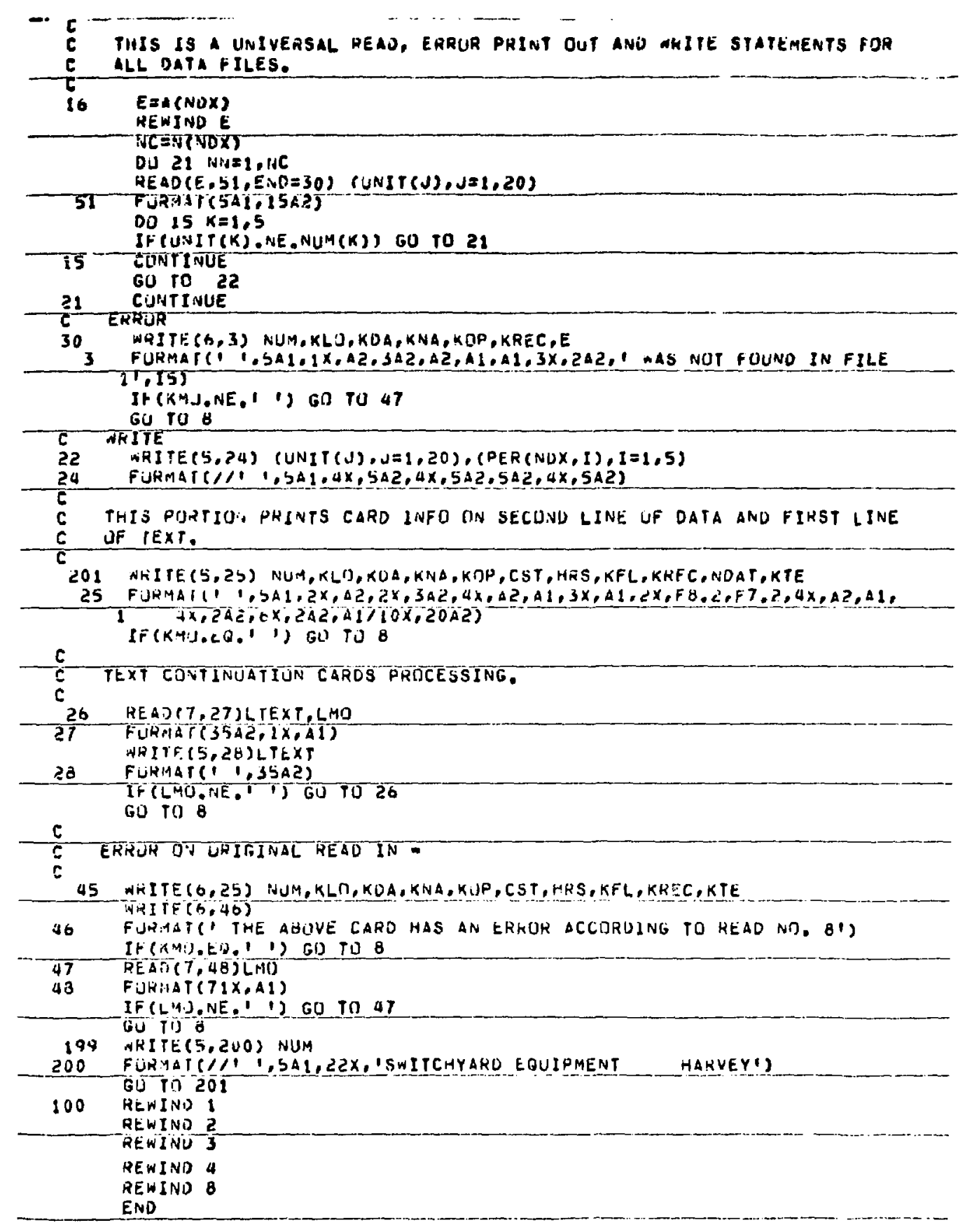

